Tôhoku Math. J.

44 (1992), 471-521

\title{
QUANTUM MULTILINEAR ALGEBRA
}

\author{
MitsuYasu Hashimoto and TAKahiro Hayashi
}

(Received August 12, 1991, revised May 7, 1992)

\begin{abstract}
We construct a quantized version of the theory of multilinear algebra, based on Jimbo's solution of Yang-Baxter equation of type $A_{N-1}^{(1)}$. Using this, we discuss the polynomial representations of quantum general linear groups.
\end{abstract}

\section{CONTENTS}

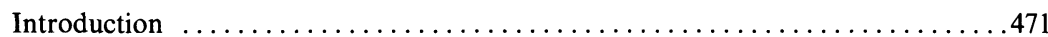

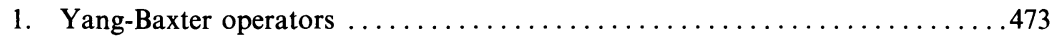

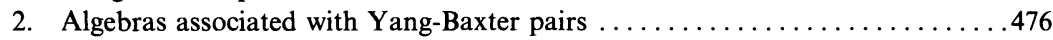

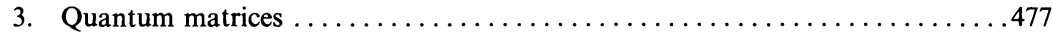

4. Symmetric algebras, exterior algebras and the fusion procedure $\ldots \ldots \ldots \ldots 480$

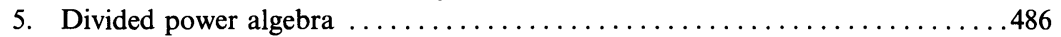

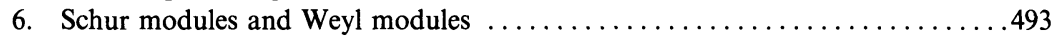

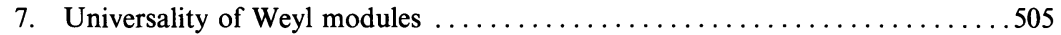

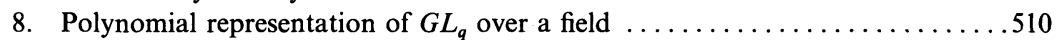

9. Quantum determinants and straightening formulas $\ldots \ldots \ldots \ldots \ldots \ldots \ldots 5$

References ........................................... 519

Introduction. Quantum groups are mathematical objects which arose from the study of the quantum inverse scattering method, especially the Yang-Baxter equation. They are very remarkable Hopf algebras and can be considered as $q$-analogues of Kac-Moody enveloping algebras or of coordinate rings of Lie groups. Not only have they added new aspects to representation theory, but also they have brought to non-commutative geometry a remarkable progress, i.e. the discovery of many new examples such as quantum linear algebraic groups, quantum spheres and so on.

In this article, we study quantum analogues of some linear-algebraic objects such as matrices, symmetric and alternating tensors, and determinants. We construct these from Jimbo's solution of Yang-Baxter (YB) equation of type $A_{N-1}^{(1)}$ and investigate their structure via the notion which we call Yang-Baxter bialgebras. As applications, we give realizations and free bases of Weyl modules $K_{\lambda} V$ and their dual modules (Schur modules) of quantum general linear groups $G L_{q}(N)$, and give a criterion for the irreducibility of $K_{\lambda} V$. We also give an analogue of the straightening formula for quantum matric bialgebras. We would like to emphasize that these objects are defined over any commutative ring $R$ and any unit element $q \in R^{\times}$and are compatible with extensions

1991 Mathematical Subject Classification. Primary 16W30. 
of the base ring $R$. Hence, we can get the representation theory of quantum general group 'defined over $Z\left[Q, Q^{-1}\right]$ ', where $Q$ denotes an indeterminate.

In Section 1, we introduce operations on YB operators (i.e. solutions of the YB equation) called the product $\times$, dual ${ }^{\vee}$, and fusion procedure. In Section 2 , we associate with a YB operator two algebras which we call the symmetric and the exterior algebras. In Section 3, we apply these tools to the construction of bialgebras $\mathrm{SE}^{\vee}$ which are called quantum matric bialgebras (cf. [12]).

In Section 4, we construct two YB operators $\varphi$ and $\psi$ on the symmetric and exterior algebras of Jimbo's YB operators of type $A_{N-1}^{(1)}$ using the fusion procedure. With the YB operator $\varphi$, we introduce unusual algebra structures into tensor products of these algebras, and prove that these algebras have some structures which we call YB-bialgebras. Though a YB-bialgebra has structures of an algebra and a coalgebra, it is not necessarily a bialgebra in the usual sense. The 'commutativity' and the 'cocommutativity' of these YB-bialgebras are described in terms of the YB-operator $\psi$.

In Section 5, we discuss an analogue of the theory of graded multilinear bialgebras. Recall that divided power algebras have been important in the study of characteristicfree representation theory (cf. [2], [3], [4]). Here we introduce divided power algebras of the YB pairs treated in Section 4. They are defined to be graded duals of symmetric algebras defined in Section 4. This concept enables us to define Weyl modules and Schur algebras in a natural way without assuming that $q$ is not a root of unity.

From Sections 6 to 9, our interest is concentrated on the study of the representation theory of quantum deformations of general linear groups.

In Section 6, we define (deformed) Weyl modules $K_{\lambda} V$ and Schur modules $L_{\lambda} V$ associated to a partition $\lambda$, using the YB-bialgebra structure of the symmetric and exterior algebras defined in Section 4 . We prove that $L_{\lambda} V$ and $K_{\lambda} V$ are finite free $R$-modules and that they are 'defined over $Z\left[Q, Q^{-1}\right]$ ' in the sense that they are compatible with base extensions. This property is an analogue of the so-called universal freeness. For the original version of this result, we refer the reader to [4]. Though our definition of $L_{\lambda} V$ is different from $L_{q}^{\lambda}(B)$ in Taft-Towber [37], they will turn out to be equivalent (cf. Proposition 9.7). Though our construction and argument in this section are nothing but the deformed versions of those in [4, Chapter II], we include some details, since they do not seem to be so obvious.

In Section 7, we introduce the deformed versions of the Schur algebra, weights, and the contravariant dual functor in our language. We will show that (deformed) Weyl modules are universal highest weight modules in the sense of Theorem 7.12.

In Section 8, we work over a base field $K$, and discuss the irreducibility and complete reducibility of $S E^{\vee}$-comodules. The notion of formal characters is introduced in this section. Theorem 8.9 is a $q$-analogue of the hook length criterion for the irreducibility of Weyl modules. In the proof, Gyoja's $q$-analogues of Young symmetrizers [14] play important roles.

In Section 9, we prove a $q$-analogue of the straightening formula using quantum de- 
terminants. Namely, we prove that the degree $k$ component $S_{k} E^{\vee}$ of $S E^{\vee}$ admits a filtration of $S E^{\vee}$-subbicomodules whose associated graded object is $\bigoplus_{|\lambda|=k}\left(K_{\lambda} V\right)^{*} \otimes L_{\tilde{\lambda}} V$, where $\tilde{\lambda}$ is the transpose of $\lambda$. This formula was originated by Doubilet-Rota-Stein [11] in the case $q=1$. Our approach is a $q$-analogue of the treatment in [4].

Lastly, we remark on the relation between our construction and the quantum enveloping algebra $U_{q} \mathrm{gl}(N)$, or rather the "quantum hyperalgebra" such as that defined in Lusztig [23]. Since these Hopf algebras satisfy the conditions in Theorem 3.3, there are pairings of bialgebras between the quantum matrix bialgebra over Jimbo's YB pair of type $A_{N-1}^{(1)}$ and the quantum hyperalgebra. Hence, thanks to the general theory of bialgebras, the quantum hyperalgebra acts on our Schur and Weyl modules. Moreover, the criterion for the irreducibility (Theorem 8.9) is also valid when they are viewed as representations over the quantum hyperalgebra.

After the submission of the first version of this work, the authors received preprints by Dipper-James [10], Noumi-Yamada-Mimachi [29], Parshall-Wang [30], which have some overlap with our paper.

Our special thanks are due to Professors Mitsuo Hoshino, Yukihiro Kanie, Hideyuki Matsumura and Akihiro Tsuchiya for valuable advice.

1. Yang-Baxter operators. Let $V$ be a free $R$-module. A Yang-Baxter (or YB) operator on $V$ is an automorphism $\beta_{V} \in \operatorname{End}_{R}(V \otimes V)$ such that

$$
\begin{aligned}
& \left(\beta_{V}\right)_{1} \circ\left(\beta_{V}\right)_{2} \circ\left(\beta_{V}\right)_{1}=\left(\beta_{V}\right)_{2} \circ\left(\beta_{V}\right)_{1} \circ\left(\beta_{V}\right)_{2}, \\
& \left(\beta_{V}\right)_{1}:=\beta_{V} \otimes \mathrm{id}_{V}, \quad\left(\beta_{V}\right)_{2}:=\mathrm{id}_{V} \otimes \beta_{V} .
\end{aligned}
$$

A Yang-Baxter pair $V=\left(V, \beta_{V}\right)$ is a free $R$-module $V$ equipped with a Yang-Baxter operator $\beta_{V}$ on $V$.

Here we give some examples of Yang-Baxter pairs.

ExAmple (1) (trivial twisting). Let $V$ be a free $R$-module. Then the map $\tau_{V}:=$ $\tau_{V, V}$ defined by $\tau_{V}\left(u \otimes u^{\prime}\right)=u^{\prime} \otimes u$ is a YB operator on $V$. We call $\tau_{V}$ the trivial twisting on $V$.

EXAMPLE (2) (Jimbo's operators of type $A_{N-1}^{(1)}$ ). Let $V$ be a free $R$-module with a basis $\left\{u_{1}, u_{2}, \ldots, u_{N}\right\}$ and let $q$ be an invertible element of $R$. Then Jimbo's YB operators of type $A_{N-1}^{(1)}$ is the map defined by the following formula:

$$
\beta_{V}\left(u_{i} \otimes u_{j}\right)= \begin{cases}u_{i} \otimes u_{i} & (i=j), \\ q u_{j} \otimes u_{i} & (i<j), \\ \left(1-q^{2}\right) u_{i} \otimes u_{j}+q u_{j} \otimes u_{i} & (i>j) .\end{cases}
$$

We call $\left\{u_{i}\right\}$ the standard basis of $\left(V, \beta_{V}\right)$. This operator also satisfies Iwahori's quadratic equation

$$
\left(\mathrm{id}_{V \otimes V}-\beta_{V}\right) \circ\left(\mathrm{id}_{V \otimes V}+q^{-2} \beta_{V}\right)=0 .
$$


As for Jimbo's other operators, we refer the reader to [16] and [31].

EXAMPLE (3) (A 'super symmetric extension' of Jimbo's type $A_{N-1}^{(1)}$ operator). Let $V$ be a free module with a basis $\left\{u_{1}^{0}, u_{2}^{0}, \ldots, u_{M}^{0}, u_{1}^{1}, \ldots, u_{N}^{1}\right\}$ and $q$ an invertible element of $R$ such that $q^{2}+1$ is also invertible. Then the following formulas define a YB operator $\beta_{V}$ on $V$ :

$$
\begin{aligned}
& \beta_{V}\left(u_{i}^{0} \otimes u_{i}^{0}\right)=u_{i}^{0} \otimes u_{i}^{0}, \quad \beta_{V}\left(u_{i}^{1} \otimes u_{i}^{1}\right)=-q^{2} u_{i}^{1} \otimes u_{i}^{1}, \\
& \beta_{V}\left(u_{i}^{0} \otimes u_{i}^{1}\right)=q u_{i}^{1} \otimes u_{i}^{0}, \quad \beta_{V}\left(u_{i}^{1} \otimes u_{i}^{0}\right)=\left(1-q^{2}\right) u_{i}^{1} \otimes u_{i}^{0}+q u_{i}^{0} \otimes u_{i}^{1}, \\
& \beta_{V}\left(u_{i}^{a} \otimes u_{j}^{b}\right)= \begin{cases}(-1)^{a b} q u_{j}^{b} \otimes u_{i}^{a} & (i<j) \\
\left(1-q^{2}\right) u_{i}^{a} \otimes u_{j}^{b}+(-1)^{a b} q u_{j}^{b} \otimes u_{i}^{a} & (i>j) .\end{cases}
\end{aligned}
$$

The restriction of this operator to $\left(\oplus_{i} R u_{i}^{0}\right)^{\otimes 2}$ coincides with the operator in Example (2) under the identification $u_{i}^{0}=u_{i}$. This operator also satisfies the relation (1.3).

EXAMPLE (4) (YB operators associated with distributive lattices). Let $(L, \wedge, \vee)$ be a distributive lattice, i.e. a set together with two maps $\wedge, \vee: L \times L \rightarrow L$ satisfying the following laws:

$$
\begin{aligned}
& a \wedge b=b \wedge a, \quad a \vee b=b \vee a, \quad a \wedge(b \wedge c)=(a \wedge b) \wedge c, \quad a \vee(b \vee c)=(a \vee b) \vee c, \\
& a \vee(a \wedge b)=(a \vee b) \wedge a=a, \quad a \wedge(b \vee c)=(a \wedge b) \vee(a \wedge c) \quad(a, b, c \in L) .
\end{aligned}
$$

Let $V$ be the free $R$-module on $L$ and define $\beta_{V} \in \operatorname{End}_{R}(V \otimes V)$ by $\beta_{V}(a \otimes b)=a \wedge b \otimes a \vee b$ $(a, b \in L)$. Then $\beta_{V}$ satisfies the condition for a YB operator except the existence of $\beta_{V}^{-1}$.

Let $\mathfrak{B}_{k}$ be the E. Artin's braid group. Namely, $\mathfrak{B}_{\boldsymbol{k}}$ is a group generated by elements $\left\{b_{i} \mid 1 \leq i<k\right\}$ with the following fundamental relations:

$$
b_{i} b_{j}=b_{j} b_{i} \quad(|i-j| \geq 2), \quad b_{i} b_{i+1} b_{i}=b_{i+1} b_{i} b_{i+1} \quad(1 \leq i \leq k-2) .
$$

For each YB pair $\left(V, \beta_{V}\right)$, there is a representation of $\mathfrak{B}_{k}$ on the $k$-fold tensor product $T_{k} V=V^{\otimes k}$ defined by

$$
b_{i} \mapsto\left(\beta_{V}\right)_{i}:=\left(\mathrm{id}_{V}\right)^{\otimes i-1} \otimes \beta_{V} \otimes\left(\mathrm{id}_{V}\right)^{\otimes k-i-1} .
$$

There is an important observation which is due to Iwahori in the Coxeter group case. Let $\mathfrak{\Xi}_{k}$ be the symmetric group of degree $k$. The length $\ell(\sigma)$ of $\sigma \in \Im_{k}$ is the number of the pairs $(i, j)$ such that $1 \leq i<j \leq k$ and $\sigma i>\sigma j$. It is well-known that $\Im_{k}$ is generated by the transpositions $\sigma_{i}=(i, i+1)(1 \leq i<k)$ and that $\ell(\sigma)$ coincides with the minimal number of factors needed to express $\sigma$ as a product of the $\sigma_{i}$ 's. An expression $\sigma=s_{1} s_{2} \cdots s_{i}$ $\left(s_{1}, \ldots, s_{i} \in \mathfrak{\Xi}_{k}\right)$ of $\sigma \in \mathfrak{\Xi}_{k}$ is said to be reduced if $\ell(\sigma)=\ell\left(s_{1}\right)+\cdots+\ell\left(s_{i}\right)$. Then Iwahori's theorem says that there is a well-defined map $\mathfrak{\Xi}_{k} \rightarrow \mathfrak{B}_{k}$ which sends $\sigma \in \mathfrak{\Xi}_{k}$ in reduced expression $\sigma=\sigma_{i_{1}} \sigma_{i_{2}} \cdots \sigma_{i_{\ell(\sigma)}}\left(1 \leq i_{1}, \ldots, i_{\ell(\sigma)} \leq k\right)$ to the element $b_{i_{1}} b_{i_{2}} \cdots b_{i_{\ell(\sigma)}}$ of the braid group. Combining this map with the homomorphism (1.7), we get a map from $\mathfrak{\Xi}_{k}$ into $\operatorname{End}_{R}\left(T_{k} V\right)$. We denote the image of $\sigma \in \Im_{k}$ by $\beta_{V}(\sigma)$. Note that if $\sigma=s_{1} \cdots s_{i}\left(s_{1}, \ldots\right.$, 
$\left.s_{i} \in \Im_{k}\right)$ is reduced, then $\beta_{V}(\sigma)=\beta_{V}\left(s_{1}\right) \cdots \beta_{V}\left(s_{i}\right)$. Let $\chi_{i j}(i, j \geq 1)$ be an element of $\mathfrak{S}_{i+j}$ defined by

$$
\chi_{i j}=\left(\begin{array}{ccccccccc}
1 & 2 & 3 & \cdots & i & i+1 & i+2 & \cdots & i+j \\
j+1 & j+2 & j+3 & \cdots & j+i & 1 & 2 & \cdots & j
\end{array}\right) .
$$

Then, $\beta_{V}\left(\chi_{i j}\right)$ defines a linear isomorphism from $T_{i} V \otimes T_{j} V$ onto $T_{j} V \otimes T_{i} V$. By Iwahori's theorem we get:

Proposition 1.1 (fusion procedure). The R-endomorphism $T \beta_{V}:=\bigoplus_{i, j \geq 0} \beta_{V}\left(\chi_{i j}\right)$ (resp. $\left.T_{i} \beta_{V}:=\beta_{V}\left(\chi_{i i}\right)\right)$ defines a YB operator on the tensor algebra $T V=\bigoplus_{k \geq 0} T_{k} V$ (resp. $\left.T_{i} V\right)$.

We denote the YB pair $\left(T V, T \beta_{V}\right)\left(\operatorname{resp} .\left(T_{i} V, T_{i} \beta_{V}\right)\right)$ by $T\left(V, \beta_{V}\right)\left(\operatorname{resp} . T_{i}\left(V, \beta_{V}\right)\right)$.

Now we will define a category $\mathscr{Y} \mathscr{B}_{R}$ as follows:

(1) The objects of $\mathscr{Y} \mathscr{B}_{R}$ consist of all YB pairs on free $R$-modules of finite rank.

(2) For Yang-Baxter pairs $V=\left(V, \beta_{V}\right)$ and $W=\left(W, \beta_{W}\right)$, the set of morphisms $\mathscr{Y} \mathscr{B}_{R}(V, W)$ consists of all $R$-module maps $f: V \rightarrow W$ satisfying the following commutative diagram:

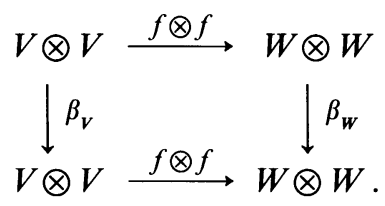

It is easy to see that $T_{i}$ is a functor from $\mathscr{Y} \mathscr{B}_{R}$ to itself for $i \geq 0$.

Now we will give more operations on $\mathscr{Y}_{\mathscr{B}_{R}}$. Let $V=\left(V, \beta_{V}\right)$ and $W=\left(W, \beta_{W}\right)$ be Yang-Baxter pairs.

DEFINITION 1.2. The product $\beta_{V} \times \beta_{W}$ of $\beta_{V}$ and $\beta_{W}$ is a YB operator on $V \otimes W$ defined by the following commutative diagram:

$$
\begin{array}{ccc}
V^{\otimes 2} \otimes W^{\otimes 2} & \stackrel{1 \otimes \tau \otimes 1}{\longrightarrow} V \otimes W \otimes V \otimes W \\
{ }_{\beta_{V} \otimes \beta_{W}} & & \beta_{V} \times \beta_{W} \\
V^{\otimes 2} \otimes W^{\otimes 2} & \stackrel{1 \otimes \tau \otimes 1}{\longrightarrow} V \otimes W \otimes V \otimes W .
\end{array}
$$

We denote the corresponding YB-pair by $V \times W$.

PROPOSITION 1.3. The product defines a bifunctor $\mathscr{Y}_{\mathscr{B}_{R}} \times \mathscr{Y} \mathscr{B}_{R} \rightarrow \mathscr{Y} \mathscr{B}_{R}$ satisfying the following conditions:

(1) associativity constraint

$$
(U \times V) \times W=U \times(V \times W)
$$


(2) existence of the identity object

$$
\left(R, \mathrm{id}_{R \otimes R}\right) \times V=V \times\left(R, \mathrm{id}_{R \otimes R}\right)=V
$$

(3) commutativity constraint

$$
\tau_{V, W}: V \times W \simeq W \times V .
$$

The proof is straightforward.

DEFINITION 1.4. Let $\left(V, \beta_{V}\right)$ be an object of $\mathscr{Y}_{\mathscr{B}_{R}}$. We define a YB pair ${ }^{t} V=\left(V^{*},{ }^{t} \beta_{V}\right)\left(\right.$ resp. $V^{-1}$, resp. $\left.V^{\vee}=\left(V^{*}, \beta_{V}^{\vee}\right)\right)$ by

$$
\operatorname{ev}\left({ }^{t} \beta_{V}\left(v \otimes v^{\prime}\right) \otimes\left(u \otimes u^{\prime}\right)\right)=\operatorname{ev}\left(\left(v \otimes v^{\prime}\right) \otimes \beta_{V}\left(u \otimes u^{\prime}\right)\right) \quad\left(v, v^{\prime} \in V^{*}, w, w^{\prime} \in V\right) .
$$

(resp. $V^{-1}=\left(V, \beta_{V}^{-1}\right)$, resp. $\left.\beta_{V}^{\vee}={ }^{t}\left(\beta_{V}^{-1}\right)\right)$ and call it the transpose (resp. inverse, resp. dual) of $V$.

It is important that the evaluation map ev: $V^{*} \otimes V \rightarrow R$ gives a morphism of $\mathscr{Y}_{\mathscr{B}_{R}}$ from $V^{\vee} \times V$ onto the unit YB pair $\left(R, \mathrm{id}_{R \otimes R}\right)$. The following theorem shows that $\mathscr{Y}_{\mathscr{B}_{R}}$ is a rigid tensor category (see [9]):

THEOREM 1.5. We have the following isomorphisms of functors.

(1) $\left(V^{\vee}\right)^{\vee}=V, \quad$ (2) $(V \times W)^{\vee}=V^{\vee} \times W^{\vee}$,

(3) $\mathscr{Y}_{\mathscr{B}_{R}}(U \times V, W) \simeq \mathscr{Y} \mathscr{B}_{R}\left(V, U^{\vee} \times W\right) \quad\left(U, V, W \in \mathscr{Y} \mathscr{B}_{R}\right)$.

Proof. Parts (1) and (2) are obvious. To see Part (3), we note that under the isomorphism $\operatorname{Hom}_{R}(U \otimes V, W) \simeq \operatorname{Hom}_{R}\left(V, U^{*} \otimes W\right)$, a map $f: U \otimes V \rightarrow W$ corresponds to the composite

$$
f^{\prime}: V \stackrel{\mathrm{ev}^{*} \otimes \mathrm{id}_{V}}{\longrightarrow} U^{*} \otimes U \otimes V \stackrel{\mathrm{id}_{U^{*}} \otimes f}{\longrightarrow} U^{*} \otimes W
$$

and the $\operatorname{map} f^{\prime}: V \rightarrow U^{*} \otimes W$ corresponds to the composite

$$
f: U \otimes V \stackrel{\operatorname{id}_{U} \otimes f^{\prime}}{\longrightarrow} U \otimes U^{*} \otimes W \stackrel{\operatorname{ev} \otimes \operatorname{id}_{W}}{\longrightarrow} W .
$$

Since ev* $\otimes \operatorname{id}_{V} \in \mathscr{Y} \mathscr{B}_{R}\left(V, U^{\vee} \times U \times V\right)$ and $\mathrm{ev} \otimes \mathrm{id}_{W} \in \mathscr{Y} \mathscr{B}_{R}\left(U^{\vee} \times U \times W, W\right)$, the map $f$ is a morphism of $\mathscr{Y} \mathscr{R}_{R}$ if and only if so is $f^{\prime}$. Therefore (1.12) and (1.13) give the desired isomorphim.

2. Symmetric and exterior algebras associated with Yang-Baxter pairs. In this section, we introduce two classes of algebras associated with YB pairs.

Definition 2.1. Let $V=\left(V, \beta_{V}\right)$ be a YB pair. Define quotient graded algebras $S V=\oplus_{i \geq 0} S_{i} V$ and $\bigwedge V=\oplus_{i \geq 0} \bigwedge_{i} V$ of the tensor algebra $T V=\oplus_{i \geq 0} V^{\otimes i}$ as follows: 


$$
\begin{aligned}
& S V:=T V /\left(\operatorname{Im}\left(\mathrm{id}_{V \otimes V}-\beta_{V}\right)\right), \\
& \bigwedge V:=T V /\left(\operatorname{Ker}\left(\mathrm{id}_{V \otimes V}-\beta_{V}\right)\right) .
\end{aligned}
$$

We call $S V$ and $\bigwedge V$ the symmetric and the exterior algebra of $V$, respectively.

To distinguish elements of $\bigwedge V$ with those of $S V$, we denote the product of $a, b \in \bigwedge V$ by $a \wedge b$.

If $\beta_{V}$ is the trivial twisting $\tau_{V}$, then $S V$ (resp. $\bigwedge V$ ) coincides with the usual symmetric (resp. exterior) algebra of the free $R$-module $V$. For Jimbo's YB pair of type $A_{N-1}^{(1)}$, the fundamental relations of these algebras are given by

$$
\begin{aligned}
& S V=\left\langle u_{1}, \ldots, u_{N} \mid u_{i} u_{j}=q u_{j} u_{i} \quad(i<j)\right\rangle, \\
& \wedge V=\left\langle u_{1}, \ldots, u_{N} \mid u_{i} \wedge u_{i}=0, q u_{i} \wedge u_{j}+u_{j} \wedge u_{i}=0 \quad(i<j)\right\rangle .
\end{aligned}
$$

Hence, in this case, we have:

(2.3) If $i_{1}, \ldots, i_{k} \in[1, N]$ has a repetition, then $u_{i_{1}} \wedge \cdots \wedge u_{i_{k}}=0$ in $\wedge V$.

$$
\begin{aligned}
& \text { If } 1 \leq i_{1}<\cdots<i_{k} \leq N \text { and } \sigma \in \Xi_{k} \text {, then } u_{i_{1}} \wedge \cdots \wedge u_{i_{k}}=(-q)^{-\ell(\sigma)} u_{i_{\sigma 1}} \wedge \cdots \wedge u_{i_{\sigma k}} \\
& \text { for } k \in N \text {. }
\end{aligned}
$$

The $R$-modules $S_{r} V$ and $\bigwedge_{r} V$ are free with respective bases

$$
\begin{gathered}
\left\{u_{j_{1}} u_{j_{2}} \cdots u_{j_{r}} \mid 1 \leq j_{1} \leq j_{2} \leq \cdots \leq j_{r} \leq N\right\}, \\
\left\{u_{j_{1}} \wedge u_{j_{2}} \wedge \cdots \wedge u_{j_{r}} \mid 1 \leq j_{1}<j_{2}<\cdots<j_{r} \leq N\right\} .
\end{gathered}
$$

For the YB pair associated with a distributive lattice $L$, the symmetric algebra is the commutative algebra generated by elements of $L$ with the fundamental relations $a \cdot b=(a \wedge b) \cdot(a \vee b)(a, b \in L)$. This algebra was introduced by Hibi [16] to study lattices by means of commutative ring theory.

3. Quantum matrices. Let $V$ be a finite free $R$-module. Then, the dual coalgebra of $\operatorname{End}(V)$ is identified with $V^{*} \otimes V$ as an $R$-module. With this identification, $V^{*} \otimes V$ is an $R$-coalgebra with the coproduct

$$
\bar{\delta}: V^{*} \otimes V \simeq V^{*} \otimes R \otimes V \stackrel{1 \otimes \mathrm{ev}^{*} \otimes 1}{\longrightarrow} V^{*} \otimes V \otimes V^{*} \otimes V
$$

and the counit $\bar{e}=\mathrm{ev}: V^{*} \otimes V \rightarrow R$. More explicitly, these maps are expressed as

$$
\bar{\delta}\left(x_{i j}\right)=\sum_{k=1}^{N} x_{i k} \otimes x_{k j}, \quad \bar{e}\left(x_{i j}\right)=\delta_{i j},
$$

where $\left\{u_{1}, \ldots, u_{N}\right\}$ is a free basis of $V,\left\{v_{1}, \ldots, v_{N}\right\}$ is its dual basis and $x_{i j}=v_{i} \otimes u_{j}$, and $\delta_{i j}$ is Kronecker's delta.

The $R$-module $V$ is a $V^{*} \otimes V$-comodule with the coaction 


$$
\bar{\omega}: V \simeq R \otimes V \stackrel{\mathrm{ev}^{*} \otimes 1}{\longrightarrow} V \otimes V^{*} \otimes V ; \quad u_{j} \longmapsto \sum_{i=1}^{N} u_{i} \otimes x_{i j} .
$$

The maps $\bar{\delta}$ and $\bar{e}$ are extended to the (unique) algebra maps $\delta: T\left(V^{*} \otimes V\right) \rightarrow$ $T\left(V^{*} \otimes V\right) \otimes T\left(V^{*} \otimes V\right)$ and $\bar{e}: T\left(V^{*} \otimes V\right) \rightarrow R$, respectively, and $T\left(V^{*} \otimes V\right)$ is a bialgebra. Hence, $V^{\otimes k}$ is a $T\left(V^{*} \otimes V\right)$-comodule with the coaction $\bar{\omega}$ given by

$$
\bar{\omega}\left(u_{j_{1}} \otimes \cdots \otimes u_{j_{k}}\right)=\sum_{i_{1}, \ldots, i_{k}}\left(u_{i_{1}} \otimes \cdots \otimes u_{i_{k}}\right) \otimes\left(x_{i_{1} j_{1}} \otimes \cdots \otimes x_{i_{k} j_{k}}\right) .
$$

Let $A$ be an $R$-coalgebra, and $V$ an $A$-comodule with coaction $\omega_{V}: V \rightarrow V \otimes A$. We define a coalgebra homomorphism $\mathrm{cf}_{V}: V^{*} \otimes V \rightarrow A$ by $\mathrm{cf}_{V}=\left(\mathrm{ev} \otimes \mathrm{id}_{A}\right) \circ\left(\mathrm{id}_{V^{\vee}} \otimes \omega_{V}\right)$ and call it the coefficient map of $V$. The coaction $\omega_{V}$ is determined by $\mathrm{cf}_{V}$ by the formula $\omega_{V}=\left(\mathrm{id}_{V} \otimes \mathrm{cf}_{V}\right) \circ \bar{\omega}$.

Now we consider a YB operator $\beta_{V}$ on $V$. The YB pair $\left(E, \beta_{E}\right):=\left(V, \beta_{V}\right) \times\left(V, \beta_{V}\right)^{\vee}$ is called the internal End of the YB pair $\left(V, \beta_{V}\right)$. The $R$-module $E$ (resp. $\left.E^{\vee}\right)$ is nothing but $V \otimes V^{*}$ (resp. $V^{*} \otimes V$ ). By Theorem 1.5, $E^{\vee}$ is identified with the internal End $V^{\vee} \times\left(V^{\vee}\right)^{\vee}$ of $V^{\vee}$. Since ev* $\in \mathscr{Y} \mathscr{B}_{R}\left(R, V^{\vee} \times V\right)$ and ev $\in \mathscr{Y} \mathscr{B}_{R}\left(V \times V^{\vee}, R\right)$, the maps $\bar{\delta}, \bar{e}$ and $\bar{\omega}$ are contained in $\mathscr{Y} \mathscr{B}_{R}\left(E^{\vee}, E^{\vee} \times E^{\vee}\right), \mathscr{Y} \mathscr{B}_{R}\left(E^{\vee}, R\right)$ and $\mathscr{Y} \mathscr{B}_{R}\left(V, V \times E^{\vee}\right)$, respectively. It is easy to see that $\beta_{E^{\vee}}$ is a coalgebra map. Hence, by [34, Proposition 1.4.8], $\left(\operatorname{Im}\left(\mathrm{id}_{E^{\vee}} \otimes E^{\vee}-\beta_{E^{\vee}}\right)\right)$ is a bi-ideal of $T E^{\vee}$. Thus the symmetric algebra $S E^{\vee}$ becomes a quotient bialgebra of the bialgebra $\left(T E^{\vee}, \bar{\delta}, \bar{e}\right)$. We call this the quantum matric bialgebra for the YB pair $\left(V, \beta_{V}\right)$ and denote its coproduct and counit by $\delta_{S}$ and $e_{S}$, respectively. Note that the exterior algebra $\bigwedge E^{\vee}$ also becomes a quotient bialgebra of $\left(T E^{\vee}, \bar{\delta}, \bar{e}\right)$.

Let us write down the fundamental relations of $S E^{\vee}$ and $\Lambda E^{\vee}$ for Jimbo's YB pair of type $A_{N-1}^{(1)}$. For Jimbo's YB pair of type $A_{N-1}^{(1)}$, the internal End YB operator $\beta_{E^{\vee}}$ is given by

$$
\begin{aligned}
& \beta_{E}\left(x_{i k} \otimes x_{i k}\right)=x_{i k} \otimes x_{i k}, \quad \beta_{E}\left(x_{i l} \otimes x_{i k}\right)=\left(1-q^{2}\right) x_{i l} \otimes x_{i k}+q x_{i k} \otimes x_{i l}, \\
& \beta_{E}\left(x_{i k} \otimes x_{i l}\right)=q x_{i l} \otimes x_{i k}, \quad \beta_{E}\left(x_{j k} \otimes x_{i k}\right)=q^{-1} x_{i k} \otimes x_{j k}, \\
& \beta_{E}\left(x_{i k} \otimes x_{j k}\right)=\left(1-q^{-2}\right) x_{i k} \otimes x_{j k}+q^{-1} x_{j k} \otimes x_{i k}, \\
& \beta_{E}\left(x_{j l} \otimes x_{i k}\right)=\left(q^{-1}-q\right) x_{i l} \otimes x_{j k}+x_{i k} \otimes x_{j l}, \quad \beta_{E}\left(x_{j k} \otimes x_{i l}\right)=x_{i l} \otimes x_{j k}, \\
& \beta_{E}\left(x_{i l} \otimes x_{j k}\right)=-\left(q-q^{-1}\right)^{2} x_{i l} \otimes x_{j k}+x_{j k} \otimes x_{i l}+\left(q-q^{-1}\right)\left(x_{j l} \otimes x_{i k}-x_{i k} \otimes x_{j l}\right), \\
& \beta_{E}\left(x_{i k} \otimes x_{j l}\right)=-\left(q-q^{-1}\right) x_{i l} \otimes x_{j k}+x_{j l} \otimes x_{i k}, \quad(1 \leq i<j \leq N, 1 \leq k<l \leq N) .
\end{aligned}
$$

Hence, the fundamental relations of $S E^{\vee}$ are

$$
\begin{aligned}
& x_{i k} x_{i l}=q x_{i l} x_{i k}, \quad x_{i k} x_{j k}=q x_{j k} x_{i k}, \quad x_{i l} x_{j k}=x_{j k} x_{i l}, \\
& x_{j l} x_{i k}-x_{i k} x_{j l}+\left(q-q^{-1}\right) x_{i l} x_{j k}=0, \quad(1 \leq i<j \leq N, 1 \leq k<l \leq N),
\end{aligned}
$$

and those of $\bigwedge E^{\vee}$ are 


$$
\begin{array}{ll}
x_{i k} \wedge x_{i k}=0, \quad x_{i k} \wedge x_{i l}+q x_{i l} \wedge x_{i k}=0, & x_{i k} \wedge x_{j k}+q x_{j k} \wedge x_{i k}=0, \\
x_{i l} \wedge x_{j k}+x_{j k} \wedge x_{i l}-\left(q-q^{-1}\right) x_{i k} \wedge x_{j l}=0, & x_{i k} \wedge x_{j l}+x_{j l} \wedge x_{i k}=0, \\
& (1 \leq i<j \leq N, 1 \leq k<l \leq N) .
\end{array}
$$

Using [5], one can easily see that $S_{r} E^{\vee}$ and $\bigwedge_{r} E^{\vee}$ have the following $R$-free bases, respectively:

$$
\begin{aligned}
& \left\{x_{i_{1} j_{1}} x_{i_{2} j_{2}} \cdots x_{i_{r} j_{r}} \mid\left(i_{1}, j_{1}\right) \leq\left(i_{2}, j_{2}\right) \leq \cdots \leq\left(i_{r}, j_{r}\right)\right\} \\
& \left\{x_{i_{1} j_{1}} \wedge x_{i_{2} j_{2}} \wedge \cdots \wedge x_{i_{r} j_{r}} \mid\left(i_{1}, j_{1}\right)<\left(i_{2}, j_{2}\right)<\cdots<\left(i_{r}, j_{r}\right)\right\} .
\end{aligned}
$$

Here $\leq$ denotes the lexicographic ordering of $\{1, \ldots, N\}^{2}$.

In the remainder of this section, we will consider representations of the quantum matric bialgebra $S E^{\vee}$ for an arbitrary YB pair $\left(V, \beta_{V}\right)$. Since $V$ is a comodule for the bialgebra $S E^{\vee}$ with the structure map $\omega_{S}\left(u_{j}\right)=\sum_{i} u_{i} \otimes x_{i j}$, the $k$-fold tensor power $T_{k} V$ of $V$ is also an $S E^{\vee}$-comodule with the structure map

$$
\omega_{S}\left(u_{j_{1}} \otimes \cdots \otimes u_{j_{k}}\right)=\sum_{i_{1}, \ldots, i_{k}}\left(u_{i_{1}} \otimes \cdots \otimes u_{i_{k}}\right) \otimes x_{i_{1} j_{1}} \cdots x_{i_{k} j_{k}} .
$$

Proposition 3.1. The coaction of $S E^{\vee}$ on $T_{k} V$ commutes with the action of the braid group $\mathfrak{B}_{k}$. In other words, the following diagram is commutative for $1 \leq i<k$ :

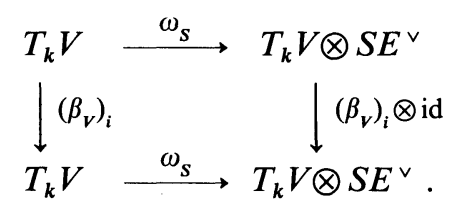

Proof. Clearly, it is enough to consider the case $k=2$. Let $\bar{\omega}$ be the coaction of $\left(T E^{\vee}, \bar{\delta}\right)$ on $T V$. Since $\bar{\omega} \in \mathscr{Y} \mathscr{B}_{R}\left(V, V \times E^{\vee}\right)$, we have

$$
\left.\left(\beta_{V} \otimes \beta_{E^{\vee}}\right) \circ \bar{\omega}\right|_{V \otimes V}=\left.\bar{\omega}\right|_{V \otimes V^{\circ}} \beta_{V}
$$

Let $p$ be the projection from $T_{2} E^{\vee}$ onto $S_{2} E^{\vee}$. Since $\left.\omega_{S}\right|_{V \otimes V}=\left.\left(\operatorname{id}_{V \otimes V} \otimes p\right) \circ \bar{\omega}\right|_{V \otimes V}$, we get

$$
\begin{aligned}
& \left.\omega_{S}\right|_{V \otimes V^{\circ}} \beta_{V}=\left.p \otimes \mathrm{id}_{V \otimes V} \circ \beta_{E} \otimes \beta_{V} \circ \bar{\omega}\right|_{V \otimes V} \\
& =\left.p \otimes \beta_{V} \circ \bar{\omega}\right|_{V \otimes V}=\left.\mathrm{id}_{S E} \otimes \beta_{V} \circ \omega_{S}\right|_{V \otimes V} .
\end{aligned}
$$

THEOREM 3.2 (Universal mapping property of $S E^{\vee}$ ). Suppose there is a bialgebra $\left(A, \delta_{A}\right)$ and coaction $\omega_{A}: V \rightarrow V \otimes A$ such that the coaction of $A$ on $T_{k} V$ commutes with the action of the braid group $\mathfrak{B}_{k}$. Then there exists a unique bialgebra homomorphism $f: S E^{\vee} \rightarrow A$ such that the following diagram is commutative. 


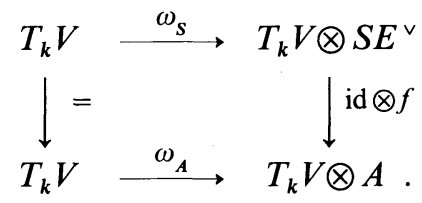

Proof. The coalgebra map $\operatorname{cf}_{V}: E^{\vee}=V^{*} \otimes V \rightarrow A$ uniquely extends to a bialgebra $\operatorname{map} \bar{f}: T E^{\vee} \rightarrow A$. By (3.10), it is easy to see that $\bar{f}\left(\operatorname{Im}\left(\mathrm{id}-\beta_{E^{\vee}}\right)\right)=0$ and that the theorem follows.

Similarly, we get:

THEOREM 3.3. Suppose there is a bialgebra $\left(U, \delta_{U}\right)$ and an action $\theta_{U}: U \otimes V \rightarrow V$ such that the action of $U$ on $T_{k} V$ commutes with the action of the braid group. Then there exists a unique bilinear pairing $\langle\rangle:, U \otimes S E^{\vee} \rightarrow R$ satisfying

(1) $\langle X, a b\rangle=(\langle, a\rangle \otimes\langle, b\rangle)\left(\delta_{U} X\right)$,

(2) $\langle X Y, a\rangle=(\langle X,\rangle \otimes\langle Y\rangle),\left(\delta_{S} a\right)$,

(3) $\theta_{U}(X \otimes w)=\left(\operatorname{id}_{T(V)} \otimes\langle X\rangle,\right)\left(\omega_{S}(w)\right) \quad\left(X, Y \in U, a, b \in S E^{\vee}, w \in T V\right)$.

In particular, the bialgebra $U$ acts on $S E^{\vee}$ by $X a=\left(\operatorname{id}_{S(E)} \otimes\langle X\rangle,\right) \circ \delta_{S}(a)(X \in U$, $a \in S E)$.

\section{Symmetric algebras, exterior algebras and the fusion procedure.}

Definition 4.1. Let $A=\left(A, m_{A}, \eta_{A}\right)$ (resp. $\left.C=\left(C, \Delta_{C}, \varepsilon_{C}\right)\right)$ be an $R$-algebra with product $m_{A}$ and unit $\eta_{A}$ (resp. an $R$-coalgebra with coproduct $\Delta_{C}$ and counit $\varepsilon_{C}$ ). Let $\varphi_{A}\left(\right.$ resp. $\left.\varphi_{C}\right)$ be a YB operator on $A$ (resp. $\left.C\right)$. We call $\left(A, \varphi_{A}\right)\left(\operatorname{resp} .\left(C, \varphi_{C}\right)\right)$ a YB algebra (resp. YB coalgebra) if the following diagrams (4.1) (resp. (4.2)) are commutative:

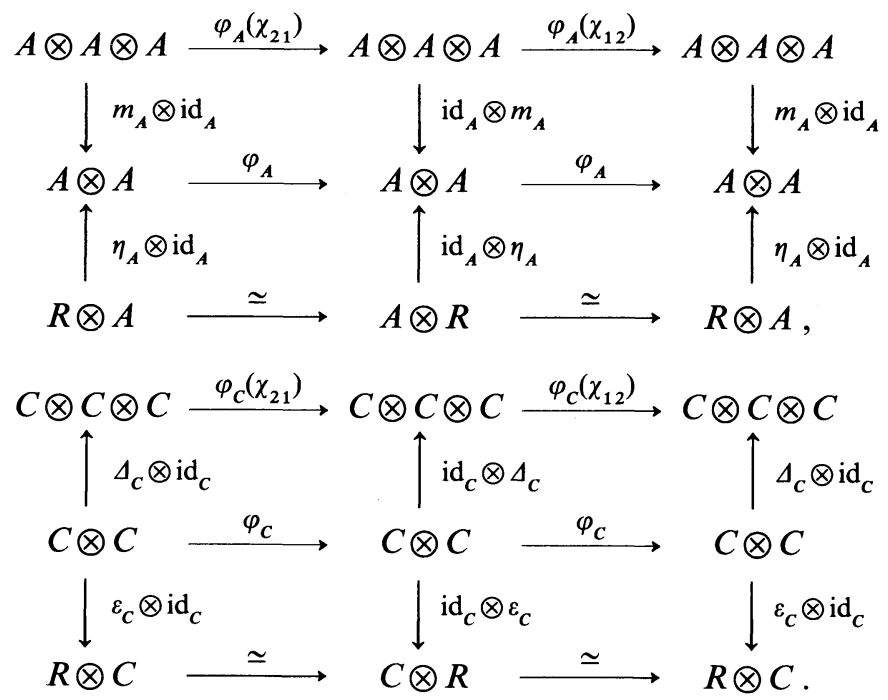


Proposition 4.2. (1) For a YB algebra $\left(A, \varphi_{A}\right)$, the YB pair $T_{i}\left(A, \varphi_{A}\right)$ (see Proposition 1.1) becomes a YB algebra with product $m_{T_{i} A}:=m_{A}^{\otimes i} \circ \varphi_{A}\left(w_{i}\right)$ and unit $\left(\eta_{A}\right)^{\otimes i}: R \simeq R^{\otimes i} \rightarrow T_{i} A$, where $w_{i}$ is the element of $\mathfrak{S}_{2 i}$ defined by

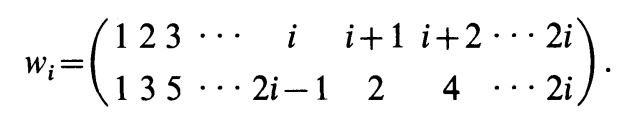

(2) For a YB coalgebra $\left(C, \varphi_{C}\right)$, the YB pair $T_{i}\left(C, \varphi_{C}\right)$ becomes a YB coalgebra with coproduct $\Delta_{T_{i} C}:=\varphi_{C}\left(w_{i}^{-1}\right) \circ \Delta_{C}^{\otimes i}$ and counit $\left(\varepsilon_{C}\right)^{\otimes i}: T_{i} C \rightarrow R^{\otimes i} \simeq R$.

Proof. By (4.1), we have

$$
\varphi_{A}\left(\chi_{i j}\right) \circ\left(m_{T_{i} A} \otimes \mathrm{id}_{A}^{\otimes j}\right)=\left(\mathrm{id}^{\otimes j} \otimes m_{A}^{\otimes i}\right) \circ \varphi_{A}\left(\chi_{2 i, j}\right) \circ\left(\varphi_{A}\left(w_{i}\right) \otimes \mathrm{id}_{A}^{\otimes j}\right) .
$$

Since both sides of the equality $\chi_{2 i, j}\left(w_{i} \times 1_{\mathfrak{S}_{j}}\right)=\left(1_{\varsigma_{j}} \times w_{i}\right) \chi_{2 i, j}$ are reduced, the right hand side of (4.4) becomes

$$
\left(\mathrm{id}^{\otimes j} \otimes m_{A}^{\otimes i}\right) \circ\left(\mathrm{id}_{A}^{\otimes j} \otimes \varphi_{A}\left(w_{i}\right)\right) \circ \varphi_{A}\left(\chi_{2 i, j}\right),
$$

hence we have

$$
\varphi_{A}\left(\chi_{i j}\right) \circ\left(m_{T_{i} A} \otimes \mathrm{id}_{A}^{\otimes j}\right)=\left(\mathrm{id}^{\otimes j} \otimes m_{T_{i} A}\right) \circ \varphi_{A}\left(\chi_{2 i, j}\right)
$$

Similarly

$$
\varphi_{A}\left(\chi_{j i}\right) \circ\left(\mathrm{id}_{A}^{\otimes j} \otimes m_{T_{i} A}\right)=\left(m_{T_{i} A} \otimes \mathrm{id}_{A}^{\otimes j}\right) \circ \varphi_{A}\left(\chi_{j, 2 i}\right) .
$$

Taking $j=i$, we find the commutativity of the upper two diagrams of (4.1) for $T_{i} A$. Now we will prove the associativity of $m_{T_{i} A}$. Since $\left(1 \times 1 \times w_{i}\right)\left(1 \times \chi_{i 1} \times 1\right)$ is a reduced expression for $w_{i+1}$, we have

$$
m_{T_{i+1} A}=\left(m_{A} \otimes m_{T_{i A}}\right) \circ\left(\mathrm{id}_{A} \otimes \varphi_{A}\left(\chi_{i 1}\right) \otimes \mathrm{id}_{T_{i} A}\right) .
$$

Hence by (4.5), (4.6), we get

$$
\begin{aligned}
& m_{T_{i+1} A^{\circ}}\left(m_{T_{i+1} A} \otimes \mathrm{id}_{T_{i+1} A}\right)=\left(\left(m_{A} \circ\left(m_{A} \otimes \mathrm{id}_{A}\right)\right) \otimes\left(m_{T_{i} A} \circ\left(m_{T_{i} A} \otimes \mathrm{id}_{T_{i} A}\right)\right)\right) \circ \varphi_{A}(\sigma), \\
& m_{T_{i+1} A^{\circ}}\left(\mathrm{id}_{T_{i+1} A} \otimes m_{T_{i+1} A}\right)=\left(\left(m_{A} \circ\left(\mathrm{id}_{A} \otimes m_{A}\right)\right) \otimes\left(m_{T_{i} A^{\circ}}\left(\mathrm{id}_{T_{i} A} \otimes m_{T_{i} A}\right)\right) \circ \varphi_{A}(\sigma),\right.
\end{aligned}
$$

where $\sigma=\left(1_{\mathfrak{S}_{2}} \times \chi_{2 i, 1} \times 1_{\mathfrak{S}_{i}}\right)\left(1_{\mathfrak{S}_{1}} \times \chi_{i 1} \times 1_{\mathfrak{G}_{2 i+1}}\right)$. Hence the associativity of $m_{T_{i+1} A}$ follows from that of $m_{A}$ and $m_{T_{i} A}$. The rest of the proof is similar and easy.

EXAMPLE. For an arbitrary algebra $A$, the YB pair $\left(A, \tau_{A}\right)$ becomes a YB algebra (cf. $\S 1$ Example (1)). The product of $T_{i}\left(A, \tau_{A}\right)$ is given by $m_{T_{i} A}\left(\left(a_{1} \otimes \cdots \otimes a_{i}\right) \otimes\left(b_{1} \otimes \cdots\right.\right.$ $\left.\left.\otimes b_{i}\right)\right)=a_{1} b_{1} \otimes \cdots \otimes a_{i} b_{i}$.

Definition 4.3. A sextuple $A=\left(A, \Delta_{A}, \varepsilon_{A}, m_{A}, \eta_{A}, \varphi_{A}\right)$ is called a graded YBbialgebra over $R$, if:

(1) $\left(A, m_{A}, \eta_{A}, \varphi_{A}\right)$ is an YB algebra over $R$ and $\left(A, \Delta_{A}, \varepsilon_{A}, \varphi_{A}\right)$ is an YB coalgebra over $R$. 
(2) The $R$-module $A$ is a direct sum of submodules $A_{i}(i \geq 0)$ such that $m_{A}\left(A_{i} \otimes A_{j}\right) \subset A_{i+j}, \Delta_{A}\left(A_{k}\right) \subset \sum_{i+j=k} A_{i} \otimes A_{j}$ and $\varphi_{A}\left(A_{i} \otimes A_{j}\right) \subset A_{j} \otimes A_{i}$.

(3) The unit $\eta_{A}$ and the counit $\varepsilon_{A}$ give an isomorphism $A_{0} \simeq R$.

(4) Both $\Delta_{A}: A \rightarrow T_{2}\left(A, m_{A}, \eta_{A}, \varphi_{A}\right)$ and $\varepsilon_{A}$ are algebra maps.

Note that in general, $A$ is not a bialgebra in the usual sense. But the following lemmas still hold.

Lemma 4.4. Under the conditions (1), (2), (3), the condition (4) in Definition 4.3 is equivalent to

(4') Both $\eta_{A}$ and $m_{A}$ are coalgebra maps, where the coalgebra structure of $A \otimes A$ is as in Proposition 4.2 (2).

LEMMA 4.5. For a graded YB bialgebra $\left(A, \varphi_{A}\right)$, the tensor product $T_{i}\left(A, \varphi_{A}\right)(i \geq 0)$ becomes a graded YB bialgebra with a product $m_{T_{i} A}$ and a coproduct $\Delta_{T_{i} A}$ (see Proposition 4.2).

Let $\left(A, \varphi_{A}\right)$ and $\left(B, \varphi_{B}\right)$ be YB algebras (resp. coalgebras, resp. bialgebras), and $f: A \rightarrow B$ a homomorphism of algebras (resp. coalgebras, resp. both algebras and coalgebras). We say that $f$ is a homomorphism of YB algebras (resp. coalgebras, resp. bialgebras) when $(f \otimes f) \circ \varphi_{A}=\varphi_{B} \circ(f \otimes f)$ (cf. (1.10)). For a homomorphism $f:\left(A, \varphi_{A}\right) \rightarrow$ $\left(B, \varphi_{B}\right)$ of $\mathrm{YB}$ algebras (resp. coalgebras, resp. bialgebras), $T_{i} f:\left(T_{i} A, T_{i} \varphi_{A}\right) \rightarrow$ $\left(T_{i} B, T_{i} \varphi_{B}\right)$ is again a homomorphism of $\mathrm{YB}$ algebras (resp. coalgebras, resp. bialgebras) for $i \geq 0$.

Let $\beta_{V}$ and $\gamma_{V}$ be YB operators on a free $R$-module $V$. We call $\left(V, \beta_{V}, \gamma_{V}\right)$ a YB triple if the following two conditions are satisfied.

$$
\begin{gathered}
\left(\mathrm{id}_{V \otimes V}-\beta_{V}\right)\left(\mathrm{id}_{V \otimes V}-\gamma_{V}\right)=\left(\mathrm{id}_{V \otimes V}-\beta_{V}\right)\left(\mathrm{id}_{V \otimes V}-\gamma_{V}\right)=0, \\
\beta_{1} \circ \beta_{2} \circ \gamma_{1}=\gamma_{2} \circ \beta_{1} \circ \beta_{2}, \quad \gamma_{1} \circ \gamma_{2} \circ \beta_{1}=\beta_{2} \circ \gamma_{1} \circ \gamma_{2}, \\
\beta_{2} \circ \beta_{1} \circ \gamma_{2}=\gamma_{1} \circ \beta_{2} \circ \beta_{1}, \quad \gamma_{2} \circ \gamma_{1} \circ \beta_{2}=\beta_{1} \circ \gamma_{2} \circ \gamma_{1} .
\end{gathered}
$$

For a YB triple $V=\left(V, \beta_{V}, \gamma_{V}\right)$, we set $S V=S\left(V, \beta_{V}\right)$ and $\bigwedge V=\bigwedge\left(V, \beta_{V}\right)$. There exist two important examples of YB triples.

EXAMPLE (1). Let $\left(V, \beta_{V}\right)$ be Jimbo's YB pair of type $A_{N-1}^{(1)}$ for a fixed ring $R$ and its invertible element $q$. By (1.3), the YB operator $\gamma_{V}:=-q^{-2} \beta_{V}$ satisfies the equation (4.9).

Example (2). Let $\left(V, \beta_{V}\right)$ be as in Example (1) and let $\left(E, \beta_{E}\right)$ be its internal End YB pair $V \times V^{\vee}$. Set $\gamma_{E}=\beta_{V} \times{ }^{t} \gamma_{V}$. It is easy to verify that $\left(E, \beta_{E}, \gamma_{E}\right)$ is a YB triple.

Let $\varphi_{A}$ and $\psi_{A}$ be YB operators on an algebra $A$. We call $\left(A, \varphi_{A}, \psi_{A}\right)$ a YB algebra triple if (1) both $\left(A, \varphi_{A}\right)$ and $\left(A, \psi_{A}\right)$ are YB algebras and (2) $\beta=\varphi_{A}$ and $\gamma=\psi_{A}$ satisfy (4.10). 
Proposition 4.6. (1) Let $\left(V, \beta_{V}\right)$ be a YB pair. Then the tensor algebra $T V=$ $\oplus_{i \geq 0} V^{\otimes i}$ becomes a graded YB bialgebra with a YB operator $T \beta_{V}$ and a coproduct $\Delta_{T V}: T V \rightarrow T_{2}\left(T V, T \beta_{V}\right)$ defined by $\Delta(u)=u \otimes 1+1 \otimes u(u \in V)$. (2) If $\left(V, \beta_{V}, \gamma_{V}\right)$ is a YB triple, then $\left(T V, T\left( \pm \beta_{V}\right), T\left( \pm \gamma_{V}\right)\right)$ is a YB algebra triple.

Proof. By Proposition 1.1, $\left(T V, T \beta_{V}\right)$ is a YB algebra. By the universal mapping property of the tensor algebra, there exists a unique algebra map $\Delta_{T V}: T V \rightarrow T_{2}\left(V, T \beta_{V}\right)$ such that $\Delta(u)=u \otimes 1+1 \otimes u$ for $u \in V$. The commutativity of (4.2) follows from Proposition 4.8 below. Next, we show the coassociativity of $\Delta_{T V}$. Because the restrictions of $\Delta_{T V} \otimes \mathrm{id} \circ \Delta_{T V}$ and $\mathrm{id} \otimes \Delta_{T V} \circ \Delta_{T V}$ on $V$ coincide, it suffices to show that these are algebra maps. Since $\Delta_{T V}$ is an algebra map, this follows easily from the commutativity of (4.2). Part (2) follows from the following lemma.

LEMMA 4.7. For a YB triple $\left(V, \beta_{V}, \gamma_{V}\right)$, we have

$$
\begin{array}{lll}
\beta\left(\chi_{i j}\right) \circ \beta_{k}=\beta_{k+j} \circ \beta\left(\chi_{i j}\right), & \gamma\left(\chi_{i j}\right) \circ \beta_{k}=\beta_{k+j} \circ \gamma\left(\chi_{i j}\right) & (1 \leq k<i), \\
\beta\left(\chi_{i j}\right) \circ \beta_{k+i}=\beta_{k} \circ \beta\left(\chi_{i j}\right), & \gamma\left(\chi_{i j}\right) \circ \beta_{k+i}=\beta_{k} \circ \gamma\left(\chi_{i j}\right) & (1 \leq k<j) .
\end{array}
$$

Proof. Since

$$
\chi_{i j}=\left(1_{\varsigma_{j-1}} \times \chi_{i 1}\right) \cdots\left(1_{\varsigma_{1}} \times \chi_{i 1} \times 1_{\Im_{j-2}}\right)\left(\chi_{i 1} \times 1_{\varsigma_{j-1}}\right)
$$

is a reduced expression, we have

$$
\gamma\left(\chi_{i j}\right)=\gamma\left(1_{\Xi_{j-1}} \times \chi_{i 1}\right) \cdots \gamma\left(1_{\Xi_{1}} \times \chi_{i 1} \times 1_{\Xi_{j-2}}\right) \gamma\left(\chi_{i 1} \times 1_{\Im_{j-1}}\right) .
$$

Hence the second identity follows from

$$
\gamma\left(1_{\mathfrak{S}_{m}} \times \chi_{i 1} \times 1_{\mathfrak{S}_{j-m-1}}\right) \beta_{k+m}=\beta_{k+m+1} \gamma\left(1_{\mathfrak{S}_{m}} \times \chi_{i 1} \times 1_{\mathfrak{S}_{j-m-1}}\right) .
$$

The other identities are obtained similarly.

To give an explicit formula for $\Delta_{T V}$, we need some notation. Let $k \geq 1$ and let $\alpha=\left(\alpha_{1}, \ldots, \alpha_{s}\right)$ be a sequence of non-negative integers with $\alpha_{1}+\cdots+\alpha_{s}=k$. Let $C=\oplus_{i \geq 0} C_{i}$ be a graded coalgebra. We denote by $\Delta_{C}^{\alpha}$ the composite map $C \rightarrow C^{\otimes s} \rightarrow C_{\alpha}$ of the $s$-th iteration of $\Delta_{C}$ and the projection, where $C_{\alpha}=C_{\alpha_{1}} \otimes \cdots \otimes C_{\alpha_{s}}$. If $\alpha$ and $C$ in consideration are clear by context, then we will drop them, and simply denote it by $\Delta$. Let $\mathfrak{\Im}_{\alpha}$ be the Young subgroup $\mathfrak{\Im}_{\alpha_{1}} \times \cdots \times \mathfrak{\Im}_{\alpha_{s}}$ of $\mathfrak{\Xi}_{k}$. We denote by $\mathfrak{\Im}^{\alpha}$ the following complete set of representatives of $\mathfrak{\Xi}_{k} / \mathfrak{\Xi}_{\alpha}$ in $\mathfrak{\Xi}_{k}$.

$$
\begin{gathered}
\Im^{\alpha}:=\left\{\sigma \in \Im_{k} \mid \sigma 1<\cdots<\sigma \alpha_{1}, \sigma\left(\alpha_{1}+1\right)<\cdots<\sigma\left(\alpha_{1}+\alpha_{2}\right), \cdots,\right. \\
\left.\sigma\left(\alpha_{1}+\cdots+\alpha_{s-1}+1\right)<\cdots<\sigma\left(\alpha_{1}+\cdots+\alpha_{s}\right)\right\} .
\end{gathered}
$$

Any $\sigma \in \mathfrak{S}_{k}$ is uniquely expressed as $\sigma=\sigma^{\prime} \sigma^{\prime \prime}$ with $\sigma^{\prime} \in \mathfrak{S}^{\alpha}$ and $\sigma^{\prime \prime} \in \mathfrak{S}_{\alpha}$, and this expression is reduced.

Proposition 4.8. For a YB pair $\left(V, \beta_{V}\right)$, the coproduct of the YB bialgebra $T\left(V, \beta_{V}\right)$ satisfies the following formula. 


$$
\Delta_{T V}^{\alpha}=\sum_{\sigma \in \mathbb{\Im}^{\alpha}} \beta_{V}\left(\sigma^{-1}\right)
$$

Proof. We prove this formula for $\alpha=(i, j)$ by induction on $k=i+j$. Let $w$ and $u$ be elements of $T_{i+j} V$ and $V$, respectively. Since $\Delta_{T V}$ is an algebra map, we have

$$
\Delta^{(i, j+1)}(w \otimes u)=m_{T_{2}(T V)}\left(\Delta^{(i-1, j+1)}(w) \otimes(u \otimes 1)\right)+m_{T_{2}(T V)}\left(\Delta^{(i, j)}(w) \otimes(1 \otimes u)\right) .
$$

By the induction hypothesis, the right hand side of (4.17) is

$$
\begin{gathered}
\left(\mathrm{id}_{V}^{\otimes i-1} \otimes \beta_{V}\left(\chi_{j+1,1}\right)\right) \circ\left(\sum_{\sigma \in \Phi^{(i-1, j+1)}} \beta_{V}\left(\sigma^{-1}\right) \otimes \mathrm{id}_{V}\right)(w \otimes u) \\
+\sum_{\sigma \in \Phi^{(i, j)}} \beta_{V}\left(\sigma^{-1}\right) \otimes \mathrm{id}_{V}(w \otimes u) .
\end{gathered}
$$

Since

$$
\mathfrak{S}^{(i, j+1)}=\left(\mathfrak{\Im}^{(i-1, j+1)}\left(1_{\mathfrak{S}_{i-1}} \times \chi_{1, j+1}\right)\right) \amalg \mathfrak{\Im}^{(i, j)},
$$

we obtain

$$
\Delta^{(i, j+1)}=\sum_{\sigma \in \Xi(i, j+1)} \beta_{V}\left(\sigma^{-1}\right)
$$

Now we fix a YB triple $V=\left(V, \beta_{V}, \gamma_{V}\right)$ such that $S_{i} V$ and $\bigwedge_{i} V$ are free $R$-modules for each $i \geq 0$. The next lemma follows easily from Lemma 4.7.

LEMMA 4.9. (1) There exist YB operators $\varphi_{S V}$ and $\psi_{S V}$ on $S V\left(\right.$ resp. $\varphi_{\wedge V}$ and $\psi_{\wedge V}$ on $\bigwedge V$ ) which satisfy

$$
\begin{array}{ll}
\varphi_{S V^{\circ}}(p \otimes p)=(p \otimes p) \circ T\left(-\gamma_{V}\right), & \psi_{S V^{\circ}}(p \otimes p)=(p \otimes p) \circ T \beta_{V}, \\
\varphi_{\wedge} V^{\circ}(p \otimes p)=(p \otimes p) \circ T\left(-\beta_{V}\right), & \psi_{\wedge V^{\circ}(p \otimes p)=(p \otimes p) \circ T \gamma_{V},},
\end{array}
$$

where $p$ denotes the projection from $T V$ onto $S V$ (resp. $\wedge V$ ).

(2) Both $\left(S V, \varphi_{S V}, \psi_{S V}\right)$ and $\left(\bigwedge V, \varphi_{\wedge}, \psi_{\wedge}\right)$ are $\mathrm{YB}$ algebra triples.

Let $A$ be either $S V$ or $\bigwedge V$. Then we have the following:

THEOREM 4.10. (1) There exists a unique algebra map $\Delta_{A}: A \rightarrow A \otimes A$ such that $\Delta_{A}(u)=u \otimes 1+1 \otimes u$ for $u \in A_{1} \simeq V$, where the algebra structure of $A \otimes A$ is given by Proposition 4.2 in terms of the YB operator $\varphi_{A}$.

(2) Define a map $\varepsilon_{A}: A \rightarrow R$ by $\varepsilon_{A}\left(\sum_{i} a_{i}\right)=a_{0}\left(a_{i} \in A_{i}\right)$. Then $\left(A, m_{A}, \eta_{A}, \Delta_{A}, \varepsilon_{A}, \varphi_{A}\right)$ becomes a graded $\mathrm{YB}$ bialgebra.

Proof. We prove this theorem only in the case $A=S V$. By Proposition 4.6 (1), it suffices to show that the ideal $\left(\operatorname{Im}\left(\mathrm{id}-\beta_{V}\right)\right)$ is a coideal of $T V$. By (4.11), 
$\left(\operatorname{Im}\left(\mathrm{id}-\beta_{V}\right)\right) \otimes T V+T V \otimes\left(\operatorname{Im}\left(\mathrm{id}-\beta_{V}\right)\right)$ is a two-sided ideal of $T_{2}\left(T V, T\left(-\gamma_{V}\right)\right)$. On the other hand, by (4.9) and (4.16),

$$
\begin{aligned}
\Delta_{T V}\left(\left(\mathrm{id}-\beta_{V}\right)(w)\right)= & \left(\mathrm{id}-\beta_{V}\right)(w) \otimes 1+1 \otimes\left(\mathrm{id}-\beta_{V}\right)(w) \\
& \in\left(\operatorname{Im}\left(\mathrm{id}-\beta_{V}\right)\right) \otimes T V+T V \otimes\left(\operatorname{Im}\left(\mathrm{id}-\beta_{V}\right)\right)
\end{aligned}
$$

for $w \in T_{2} V$.

Proposition 4.11. Let $A$ be as above. Then, (1) $m_{A} \circ \psi_{A}=m_{A}$. (2) If $\Delta: A_{i} \rightarrow A_{1}^{\otimes i}$ is injective for any $i \geq 0$, then, $\psi_{A} \circ \Delta_{A}=\Delta_{A}$.

Proof. We show this proposition only for $A=S V$. Using (4.2), we obtain

$$
\begin{aligned}
\Delta^{\left(1^{i}\right)} \otimes \Delta^{\left(1^{j}\right)} \circ \psi_{S V^{\circ}} \Delta^{(i, j)} \circ p_{i+j} & =\beta\left(\chi_{i j}\right) \circ \Delta^{\left(1^{i}\right)} \otimes \Delta^{\left(1^{j}\right)} \circ \Delta^{(i, j)} \circ p_{i+j} \\
& =\beta\left(\chi_{i j}\right) \circ \Delta^{\left(1^{i+j}\right)} \circ p_{i+j},
\end{aligned}
$$

where $\left(1^{i}\right)=(1, \ldots, 1) \in Z^{i}$. By (4.16), the right hand side of (4.19) is $\beta\left(\chi_{i j}\right) \circ \sum_{\sigma \in \varsigma_{k}}\left(-\gamma_{V}\right)(\sigma)$. Since

$$
\left(\beta_{V}\right)_{r} \sum_{\sigma \in \Xi_{k}}\left(-\gamma_{V}\right)(\sigma)=\left(\beta_{V}\right)_{r} \sum_{\ell(\sigma)<\ell((r, r+1) \sigma)}\left(\mathrm{id}-\left(\gamma_{V}\right)_{r}\right)\left(-\gamma_{V}\right)(\sigma)=\sum_{\sigma \in \Xi_{k}}\left(-\gamma_{V}\right)(\sigma)
$$

for $1 \leq r \leq i+j$, we get

$$
\Delta^{\left(1^{i}\right)} \otimes \Delta^{\left(1^{j}\right)} \circ \psi_{S V^{\circ}} \Delta^{(i, j)} \circ p_{i+j}=\Delta^{\left(1^{i}\right)} \otimes \Delta^{\left(1^{j}\right)} \circ \Delta^{(i, j)} \circ p_{i+j} .
$$

This proves Part (2). Part (1) follows from

$$
m_{A} \circ \psi_{A} \circ p_{i} \otimes p_{j}=m_{A} \circ p_{j} \otimes p_{i} \circ \beta\left(\chi_{i j}\right)=p_{i+j} \circ \beta\left(\chi_{i j}\right)=p_{i+j}=m_{A} \circ p_{i} \otimes p_{j} .
$$

Now we shall look at the representation-theoretical aspects of the YB algebras we discussed so far.

Definition 4.12. Let $H$ be an $R$-bialgebra, and $A=\left(A, \varphi_{A}\right)$ be a YB algebra with a $H$-comodule structure. We say that $A$ is $H$-equivariant $\mathrm{YB}$ algebra when $m_{A}, \eta_{A}$ and $\varphi_{A}$ are homomorphisms of $H$-comodules. We define $H$-equivariance of YB algebra triples, YB coalgebras, and YB bialgebras similarly. All of the structure morphisms (including YB operators) are required to be homomorphisms of $H$-comodules.

If $\left(A, \varphi_{A}\right)$ is a $H$-equivariant YB algebra (resp. coalgebra, resp. bialgebra), then $T_{i}\left(A, \varphi_{A}\right)$ is also $H$-equivariant for any $i \geq 0$.

Let $(V, \beta, \gamma)$ be a YB triple such that $S V$ and $\bigwedge V$ are free, that $V$ is a $H$-comodule, and that $\beta$ is a homomorphism of $H$-comodules. Then it is easy to see that $(T V, T( \pm \beta))$ is a $H$-equivariant YB algebra. The map $V \rightarrow V \otimes R \oplus R \otimes V ; u \mapsto u \otimes 1+1 \otimes u$ is clearly a $H$-homomorphism. Since $T_{2}(T V, T( \pm \beta))$ is a $H$-equivariant YB algebra, the coproduct $\Delta_{T V}$ of $(T V, T( \pm \beta))$ is also a $H$-homomorphism. Hence, $(T V, T( \pm \beta))$ is a $H$-equivariant 
YB bialgebra. Since the ideals $(\operatorname{Im}(1-\beta))$ and $(\operatorname{Ker}(1-\beta))$ are subcomodules of $T V$, we see that $S V$ and $\bigwedge V$ are quotient comodules of $T V$. The YB bialgebra structure of $(\bigwedge V, \varphi \wedge V)$ is induced by the structure of $(T V, T(-\beta))$. Hence, $\bigwedge V$ is also a $H$-equivariant YB bialgebra. Though $m_{S V}, \eta_{S V}, \varepsilon_{S V}$, and $\psi_{S V}$ are $H$-homomorphisms, $\Delta_{S V}, \varphi_{S V}$ and $\psi_{\wedge V}$ may not be $H$-homomorphisms.

The most important example of $H$ is the quantum matric bialgebra $S E^{\vee}$ (see Proposition 3.1 and Theorem 3.2). If $\gamma$ is also a $H$-homomorphism, then $(T V, T( \pm \gamma))$ is also a $H$-equivariant YB bialgebra. It is easy to see that $\left(\bigwedge V, \varphi_{\wedge V}, \psi_{\wedge V}\right)$ and $\left(S V, \varphi_{S V}, \psi_{S V}\right)$ are $H$-equivariant both as YB algebra triples and as YB bialgebras in this case. Jimbo's YB triple of type $A_{N-1}^{(1)}$ and its matric bialgebra satisfy this condition, since $\gamma_{V}=-q^{-2} \beta_{V}$.

5. Divided power algebra. For a graded $R$-module $M=\oplus_{k \geq 0} M_{k}$, the graded dual $M^{\dagger}$ of $M$ is $\oplus_{k \geq 0} M^{*}$ by definition. The graded dual $f^{\dagger}$ of a homogeneous map $f: M \rightarrow N=\oplus_{k} N_{k}$ is defined to be $\sum_{k} f_{k}^{*}$, where $f_{k}$ is the degree $k$ component of $f$.

Let $A=\left(A, \Delta_{A}, \varepsilon_{A}, m_{A}, \eta_{A}, \varphi_{A}\right)$ be a graded YB-bialgebra with each homogeneous component $A_{i}$ being free of finite rank for each $i \geq 0$. We introduce into $A^{\dagger}$ a structure of a graded YB-bialgebra. There is a canonical isomorphism $A^{\dagger} \otimes A^{\dagger} \simeq(A \otimes A)^{\dagger}$ via the pairing

$$
A_{i}^{*} \otimes A_{j}^{*} \otimes A_{i} \otimes A_{j} \stackrel{1 \otimes \tau \otimes 1}{\longrightarrow} A_{i}^{*} \otimes A_{i} \otimes A_{j}^{*} \otimes A_{j} \stackrel{\mathrm{ev} \otimes \mathrm{ev}}{\longrightarrow} R \otimes R=R,
$$

where $\tau$ is the trivial twisting. The structure morphisms $\Delta_{A^{\dagger}}, \varepsilon_{A^{+}}, m_{A^{\dagger}}, \eta_{A^{+}}$and $\varphi_{A^{\dagger}}$ are defined to be $m_{A}^{\dagger}, \eta_{A}^{\dagger}, \Delta_{A}^{\dagger}, \varepsilon_{A}^{\dagger}$ and $\varphi_{A}^{\dagger}$, respectively. For example,

$$
A_{i}^{*} \otimes A_{j}^{*} \otimes A_{j} \otimes A_{i} \stackrel{\varphi_{A^{+}} \otimes 1}{\longrightarrow} A_{j}^{*} \otimes A_{i}^{*} \otimes A_{j} \otimes A_{i} \stackrel{\text { ev }}{\longrightarrow} R
$$

is equal to

$$
A_{i}^{*} \otimes A_{j}^{*} \otimes A_{j} \otimes A_{i} \stackrel{1 \otimes \varphi_{A}}{\longrightarrow} A_{i}^{*} \otimes A_{j}^{*} \otimes A_{i} \otimes A_{j} \stackrel{\text { ev }}{\longrightarrow} R
$$

Thus, for $k \geq 0$ and $\sigma \in \Im_{k}$, the graded transpose $\left(\varphi_{A}(\sigma)\right)^{\dagger}$ of $\varphi_{A}(\sigma)$ is equal to $\varphi_{A^{\dagger}}\left(\sigma^{-1}\right)$. With these definitions, $A^{\dagger}$ is a graded YB-bialgebra. In fact, it is well-known that $\left(A^{\dagger}, m_{A^{\dagger}}, \eta_{A^{\dagger}}\right)$ is an $R$-algebra, and that $\left(A^{\dagger}, \Delta_{A^{\dagger}}, \varepsilon_{A^{\dagger}}\right)$ is an $R$-coalgebra (see e.g., [28]). Taking the dual of the diagram (4.1), we easily see that $A^{\dagger}$ is a YB coalgebra. Similarly, taking the dual of the diagram (4.2) for $A$, we see that $A^{\dagger}$ is a YB algebra. It is clear that the conditions (2) and (3) in Definition 4.3 for $A$ imply the conditions (2) and (3) for $A^{\dagger}$, respectively. By the condition (4) in Definition 4.3 for $A$, we have the condition $\left(4^{\prime}\right)$ in Lemma 4.4 for $A^{\dagger}$.

Let $V=(V, \beta, \gamma)$ be a YB triple. We assume that $V$ is a free $R$-module of finite rank and both $S V$ and $\bigwedge V$ are free. As in Section 4, the exterior algebra $\bigwedge V=$ 
$T(V,-\beta) /(\operatorname{Ker}(1-\beta))$ and the symmetric algebra $S V=T(V,-\gamma) /(\operatorname{Im}(1-\beta))$ has a structure of YB bialgebras. By (4.16), we have:

$$
\begin{aligned}
& \Delta_{\wedge V}^{(1, \ldots, 1)}\left(w_{1} \wedge \cdots \wedge w_{k}\right)=\sum_{\sigma \in \Theta_{k}}(-\beta)\left(\sigma^{-1}\right)\left(w_{1} \otimes \cdots \otimes w_{k}\right) \\
& \Delta_{S V}^{(1, \ldots, 1)}\left(w_{1} \cdots w_{k}\right)=\sum_{\sigma \in \subseteq_{k}}(-\gamma)\left(\sigma^{-1}\right)\left(w_{1} \otimes \cdots \otimes w_{k}\right)
\end{aligned}
$$

for any $k \geq 0$ and $w_{1}, \ldots, w_{k} \in V$.

We denote the YB triple $\left(V^{*},{ }^{t} \beta,{ }^{t} \gamma\right)$ by ${ }^{t} V$, where ${ }^{t} \beta\left(\right.$ resp. $\left.{ }^{t} \gamma\right): V^{*} \otimes V^{*} \rightarrow V^{*} \otimes V^{*}$ is the transpose of $\beta$ (see Definition 1.4). We assume that $S^{t} V$ and $\wedge^{t} V$ are free. Let $A=\oplus_{k \geq 0} A_{k}$ be either $\bigwedge V$ or $S V$. Note that the $i$-fold product $m_{A}: V^{\otimes i} \hookrightarrow A^{\otimes i} \rightarrow A_{i}$ is nothing but the projection map $p: T_{i} V=V^{\otimes i} \rightarrow A_{i}$. We denote by $A^{\prime}=\bigoplus_{k \geq 0} A_{k}^{\prime}$ the YB bialgebra $\bigwedge^{t} V$ or $S^{t} V$, according as $A=\bigwedge V$ or $A=S V$. For a non-negative integer $i$, we denote the pairing $T_{i} V \otimes T_{i} V^{*} \rightarrow R ; w_{1} \otimes \cdots \otimes w_{i} \otimes \varphi_{1} \otimes \cdots \otimes \varphi_{i} \mapsto \varphi_{1}\left(w_{1}\right) \cdots \varphi_{i}\left(w_{i}\right)$ by $\mathrm{Ev}^{(i)}$. We define a pairing ev ${ }^{(i)}: A_{i} \otimes A_{i}^{\prime} \rightarrow R$ by the commutativity of the diagram

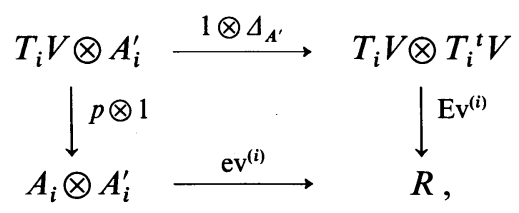

where $p: T_{i} V \rightarrow A_{i}$ is the natural projection. This pairing $\mathrm{ev}^{(i)}$ is well-defined, since $\mathrm{Ev}^{(i)}\left(\operatorname{Ker}\left(1-\beta_{l}\right) \otimes \operatorname{Im}\left(1-{ }^{t} \beta_{l}\right)\right)=0$ for $1 \leq l \leq i-1$ (the case $\left.A=\bigwedge V\right)$ and $\operatorname{Ev}^{(i)}(\operatorname{Im}(1-$ $\left.\left.\beta_{l}\right) \otimes \operatorname{Im}\left(1-{ }^{t} \gamma_{l}\right)\right)=0$ for $1 \leq l \leq i-1$ (the case $\left.A=S V\right)$.

LEMMA 5.1. In the notation above, the diagram

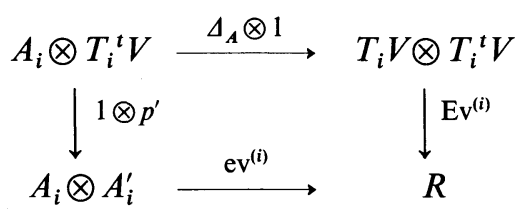

is commutative, where $p^{\prime}: T_{i}{ }^{t} V \rightarrow A_{i}^{\prime}$ is the natural projection.

Proof. First, we consider the case $A=\bigwedge V$. It suffices to show that ev ${ }^{(i)} \circ\left(p \otimes p^{\prime}\right)=$ $\mathrm{Ev}^{(i)} \circ\left(\left(\Delta_{A}^{(1, \ldots, 1)} \circ p\right) \otimes 1\right)$, since $p: T_{i} V \rightarrow A_{i}$ is surjective. By (5.1), we have

$$
\begin{aligned}
\mathrm{Ev}^{(i)} \circ\left(\left(\Delta_{A}^{(1, \ldots, 1)} \circ p\right) \otimes 1\right)=\mathrm{Ev}^{(i)} \circ\left(\left(\sum_{\sigma \in \mathcal{S}_{i}}(-\beta)\left(\sigma^{-1}\right)\right) \otimes 1\right) \\
\quad=\mathrm{Ev}^{(i)} \circ\left(1 \otimes\left(\sum_{\sigma \in \mathcal{S}_{i}}\left(-{ }^{t} \beta\right)(\sigma)\right)\right)=\mathrm{Ev}^{(i)} \circ\left(1 \otimes\left(\Delta_{A^{\prime}}^{(1, \ldots, 1)} \circ p^{\prime}\right)\right) .
\end{aligned}
$$

Since $\mathrm{Ev}^{(i)} \circ\left(1 \otimes \Delta_{A^{\prime}}^{(1, \ldots, 1)}\right)=\mathrm{ev}^{(i)} \circ(p \otimes 1)$ by the definition of $\mathrm{ev}^{(i)}$, we have completed 
the proof in this case. The case $A=S V$ is proved similarly by means of (5.2).

We denote by $\Phi_{i}^{A}$ the natural map from $A_{i}$ to $\left(A_{i}^{\prime}\right)^{*}$ induced by the pairing $\mathrm{ev}^{(i)}: A_{i} \otimes A_{i}^{\prime} \rightarrow R$, and denote by $\Phi^{A}$ the sum of $\Phi_{i}^{A}$ 's.

Proposition 5.2. In the notation above, $\Phi^{A}: A \rightarrow\left(A^{\prime}\right)^{\dagger}$ is a homomorphism of YB bialgebra. The map $\Phi^{A}$ is a surjection if and only if $\Delta_{A^{\prime}}^{\left(1^{k}\right)}$ is a split injection for any $k \geq 0$, where $\left(1^{k}\right)$ is the sequence $(1, \ldots, 1)$ ( $k$ times 1$)$.

Proof. By the definition of $\mathrm{ev}^{(i)}, \Phi=\Phi^{A}$ is a homomorphism of algebras. By Lemma 5.1, $\Phi$ is a homomorphism of coalgebras. We set $\alpha=-\beta$ when $A=\bigwedge V$, and $\alpha=-\gamma$ when $A=S V$. We show that $\varphi_{\left(A^{\prime}\right)^{\dagger \circ}}(\Phi \otimes \Phi)=(\Phi \otimes \Phi) \circ \varphi_{A^{*}}$. Since $p \otimes p: T_{i} V \otimes$ $T_{j} V \rightarrow A_{i} \otimes A_{j}$ is surjective for any $i, j \geq 0$ and

$$
\Delta_{\left(A^{\prime}\right)^{\dagger}}^{\left(1^{j}\right.} \otimes \Delta_{\left(A^{\prime}\right)^{\dagger}}^{\left(1^{i}\right)}=\left(p^{\prime}\right)^{*} \otimes\left(p^{\prime}\right)^{*}:\left(A_{j}^{\prime}\right)^{*} \otimes\left(A_{i}^{\prime}\right)^{*} \rightarrow\left(T_{j}^{t} V\right)^{*} \otimes\left(T_{i}^{t} V\right)^{*} \simeq T_{j} V \otimes T_{i} V
$$

is injective for any $i, j \geq 0$, it suffices to show

$$
\left(\left(p^{\prime}\right)^{*} \otimes\left(p^{\prime}\right)^{*}\right) \circ \varphi_{\left(A^{\prime}\right)^{\dagger} \circ}\left(\Phi_{i} \otimes \Phi_{j}\right) \circ(p \otimes p)=\left(\left(p^{\prime}\right)^{*} \otimes\left(p^{\prime}\right)^{*}\right) \circ\left(\Phi_{j} \otimes \Phi_{i}\right) \circ \varphi_{A^{\circ}}(p \otimes p)
$$

on $T_{i} V \otimes T_{j} V$ for any $i, j \geq 0$. Since $p: T_{i} V \rightarrow A_{i}$ and $p: T_{j} V \rightarrow A_{j}$ are nothing but the iterated multiplication, $\left(\Phi_{i} \otimes \Phi_{j}\right) \circ(p \otimes p)$ agrees with the composite map

$$
T_{i} V \otimes T_{j} V \simeq\left(T_{i}^{t} V\right)^{*} \otimes\left(T_{j}^{t} V\right)^{*} \stackrel{\Delta_{A^{\prime}}^{*} \otimes \Delta_{A^{\prime}}^{*}}{\longrightarrow}\left(A_{i}^{\prime}\right)^{*} \otimes\left(A_{j}^{\prime}\right)^{*},
$$

thanks to the fact that $\Phi$ is a homomorphism of algebras. We identify $\left(T_{j}{ }^{t} V\right)^{*} \otimes\left(T_{i}{ }^{t} V\right)^{*}$ with $\left(T_{j}{ }^{t} V \otimes T_{i}{ }^{t} V\right)^{*}$, and $T_{i} V \otimes T_{j} V$ with $\left(T_{i}{ }^{t} V \otimes T_{j}{ }^{t} V\right)^{*}$ in an obvious manner. Then, $\left(A_{i}^{\prime}\right)^{*} \otimes\left(A_{j}^{\prime}\right)^{*},\left(\Delta_{A^{\prime}}^{\left(1^{i}\right)}\right)^{*} \otimes\left(\Delta_{A^{\prime}}^{\left(1^{j}\right)}\right)^{*},\left(p^{\prime}\right)^{*} \otimes\left(p^{\prime}\right)^{*}$, and the restriction of $\varphi_{\left(A^{\prime}\right)^{+}}$to $\left(A_{i}^{\prime}\right)^{*} \otimes\left(A_{j}^{\prime}\right)^{*}$ are identified with $\left(A_{i}^{\prime} \otimes A_{j}^{\prime}\right)^{*},\left(\Delta_{A^{\prime}}^{(1, i)} \otimes \Delta_{A^{\prime}}^{(1, j)}\right)^{*},\left(p^{\prime} \otimes p^{\prime}\right)^{*}$, and $\left(\left(\varphi_{A^{\prime}}\right)_{A_{i}^{\prime} \otimes A_{j}^{\prime}}\right)^{*}$, respectively. So the left hand side of (5.3) is identified with the dual of the composite map $\left(\Delta_{A^{\prime}}^{\left.(1)^{i}\right)} \otimes\right.$ $\left.\Delta_{A^{\prime}}^{\left(1^{j}\right)}\right) \circ \varphi_{A^{\prime}} \circ\left(p^{\prime} \otimes p^{\prime}\right)$. By (4.18), it holds that $\varphi_{A^{\prime}} \circ\left(p^{\prime} \otimes p^{\prime}\right)=\left(p^{\prime} \otimes p^{\prime}\right) \circ T\left({ }^{t} \alpha\right)$. Since $\left(p^{\prime} \otimes p^{\prime}\right) \circ$ $\left(\Delta_{A^{\prime}}^{\left(1^{i}\right)} \otimes \Delta_{A^{\prime}}^{\left(1^{j}\right)}\right)$ is the action of $\sum_{\sigma \in \Phi_{(i, j)}}\left({ }^{t} \alpha\right)(\sigma)$ by (5.1) and (5.2), the left hand side of (5.3) agrees with the map $\left(\left(\sum_{\sigma \in \Xi_{(i, j)}}\left({ }^{t} \alpha\right)(\sigma)\right) \circ\left({ }^{t} \alpha\right)\left(\chi_{j i}\right)\right)^{*}$. On the other hand, the right hand side of $(5.3)$ is $\left(\left(\sum_{\sigma \in \mathcal{E}_{(j, i)}} \alpha(\sigma)\right) \circ \alpha\left(\chi_{i j}\right)\right)^{*}$ by a similar argument. For any element $\sigma=\sigma_{1} \times \sigma_{2} \in \Theta_{i j}$, it holds that $\left(\left(\sigma_{1} \times \sigma_{2}\right) \chi_{j i}\right)^{-1}=\left(\sigma_{2}^{-1} \times \sigma_{1}^{-1}\right) \chi_{i j}$, and these expressions are reduced. Hence, we have

$$
\left(\left(\sum_{\sigma \in \mathcal{S}_{(i, j)}}\left({ }^{t} \alpha\right)(\sigma)\right) \circ\left({ }^{t} \alpha\right)\left(\chi_{i j}\right)\right)^{*}=\left(\sum_{\sigma \in \mathcal{S}_{(i, j)}} \alpha\left(\left(\sigma \cdot \chi_{i j}\right)^{-1}\right)^{*}\right)^{*} .
$$

So (5.3) is proved, and the proof of the first assertion is complete.

Since $\Phi$ is a homomorphism of algebras, we have $\left(\Delta_{A^{\prime}}^{\left(1^{k}\right)}\right)^{*}=\Phi_{k} \circ p$ for any $k \geq 0$, where $p: T_{k} V=\rightarrow A_{k}$ is the projection, or the $k$-fold product. Hence, $\left(\Delta_{A^{\prime}}^{\left(1^{k}\right)}\right)^{*}$ is split surjective if and only if $\Phi_{k}$ is surjective, since $p$ is surjective and $\left(A_{k}^{\prime}\right)^{*}$ is free. So the second assertion is now clear. 
Lemma 5.3. Let $(V, \beta, \gamma)$ be Jimbo's YB triple of type $A_{N-1}^{(1)}$ (see Example (1) in Section 4) with the standard basis $u_{1}, \ldots, u_{N}$. Then for any $k \geq 0$ and any sequence $\left(i_{1}, \ldots, i_{k}\right)$ with $1 \leq i_{1}<\cdots<i_{k} \leq N$, we have

$$
\Delta_{\wedge V}^{(1, \ldots, 1)}\left(u_{i_{1}} \wedge \cdots \wedge u_{i_{k}}\right)=\sum_{\sigma \in \Xi_{k}}(-q)^{\ell(\sigma)} u_{i_{\sigma 1}} \otimes \cdots \otimes u_{i_{\sigma k}}
$$

In particular, $\Delta_{\wedge}: \bigwedge V \rightarrow T_{k} V$ is a split injection for any $k \geq 0$.

Proof. To prove the first assertion, it suffices to show that

$$
\beta\left(\sigma^{-1}\right)\left(u_{i_{1}} \otimes \cdots \otimes u_{i_{k}}\right)=q^{\ell(\sigma)} u_{i_{\sigma 1}} \otimes \cdots \otimes u_{i_{\sigma k}}
$$

for $\sigma \in \Im_{k}$, which is proved easily by an induction on $\ell(\sigma)$. If we define $\pi_{k}: T_{k} V \rightarrow \bigwedge_{k} V$ by

$$
\pi_{k}\left(u_{i_{1}} \otimes \cdots \otimes u_{i_{k}}\right)= \begin{cases}u_{i_{1}} \wedge \cdots \wedge u_{i_{k}} & \left(i_{1}<\cdots<i_{k}\right) \\ 0 & \text { (otherwise) }\end{cases}
$$

for $1 \leq i_{1}, \ldots, i_{k} \leq N$, then $\pi_{k} \circ \Delta_{\Lambda}^{(1, \ldots, 1)}=\mathrm{id} \wedge_{\wedge^{k} V}$. Hence, the second assertion is now clear.

Let $a$ be a non-negative integer and $t \in R$. We define $[a]_{t}=1+t+\cdots+t^{a-1}$ if $a>0$, and $[0]_{t}=0$. We also define $[a]_{t}^{!}=\prod_{i=1}^{a}[i]_{t}$ if $a>0$, and $[0]_{t}^{!}=1$.

LeMmA 5.4. Let $(V, \beta, \gamma)$ and $k$ be as in Lemma 5.3. For any sequence $\alpha=$ $\left(\alpha_{1}, \ldots, \alpha_{N}\right)$ of non-negative integers with $\sum_{i} \alpha_{i}=k$, we have

$$
\Delta_{S V}^{(1, \ldots, 1)}\left(u_{1}^{\alpha_{1}} \cdots u_{N}^{\alpha_{N}}\right)=\prod_{i=1}^{N}\left[\alpha_{i}\right]_{q^{-2}}^{!} \cdot \sum_{\sigma \in \Xi^{\alpha}} q^{-\ell(\sigma)} u_{v \sigma^{-1} 1} \otimes \cdots \otimes u_{v \sigma^{-1} k},
$$

where the number $v i$ is the $i$-th element in the sequence $\left(1^{\alpha_{1}}, \ldots, N^{\alpha_{N}}\right)$. In particular, $\Delta_{S V}: S_{k} V \rightarrow T_{k} V$ is a split injection if and only if $[a]_{q-2}$ is invertible in $R$ for any $1 \leq a \leq k$.

PROOF. It suffices to show that $\sum_{\sigma \in \subseteq_{k}}(-\gamma)(\sigma)\left(u_{1}^{\otimes \alpha_{1}} \otimes \cdots \otimes u_{N}^{\otimes \alpha_{N}}\right)$ equals the right hand side of the formula. Since $\mathfrak{S}_{k}=\mathfrak{S}^{\alpha} \cdot \mathfrak{S}_{\alpha}$, and since the expression $\sigma=\sigma^{\prime} \sigma^{\prime \prime}\left(\sigma^{\prime} \in \mathfrak{S}^{\alpha}\right.$, $\sigma^{\prime \prime} \in \mathfrak{S}_{\alpha}$ ) is reduced for any $\sigma \in \mathfrak{S}_{k}$, we have

$$
\sum_{\sigma \in \mathfrak{S}_{k}}(-\gamma)(\sigma)=\left(\sum_{\sigma^{\prime} \in \subseteq^{\alpha}}(-\gamma)\left(\sigma^{\prime}\right)\right) \cdot\left(\sum_{\sigma^{\prime \prime} \in \mathfrak{S}_{\alpha}}(-\gamma)\left(\sigma^{\prime \prime}\right)\right) .
$$

For any $a \geq 0$ and $t \in R$, it holds $\sum_{\sigma \in \subseteq_{a}} t^{\ell(\sigma)}=[a]_{t}^{!}$. Hence, it is easy to see that

$$
\sum_{\sigma^{\prime \prime} \in \subseteq_{\alpha}}(-\gamma)\left(\sigma^{\prime \prime}\right)\left(u_{1}^{\otimes \alpha_{1}} \otimes \cdots \otimes u_{N}^{\otimes \alpha_{N}}\right)=\prod_{i=1}^{N}\left[\alpha_{i}\right]_{q^{-2}}^{!} \cdot u_{1}^{\otimes \alpha_{1}} \otimes \cdots \otimes u_{N}^{\otimes \alpha_{N}}
$$

So it suffices to show that

$$
(-\gamma)\left(\sigma^{\prime}\right) u_{1}^{\otimes \alpha_{1}} \otimes \cdots \otimes u_{N}^{\otimes \alpha_{N}}=(-q)^{-\ell\left(\sigma^{\prime}\right)} u_{v \sigma^{\prime-1} 1} \otimes \cdots \otimes u_{v \sigma^{\prime-1} N},
$$


which is proved easily by induction on $\ell\left(\sigma^{\prime}\right)$.

Now assume that $[a]_{q^{-2}}$ is invertible for any $a$ such that $1 \leq a \leq k$. We define $\pi_{k}: T_{k} V \rightarrow S_{k} V$ by

$$
\pi_{k}\left(u_{i_{1}} \otimes \cdots \otimes u_{i_{k}}\right)= \begin{cases}\left(\prod_{i=1}^{N}\left[a_{i}\right]_{q^{-2}}^{!}\right)^{-1} \cdot u_{i_{1}} \cdots u_{i_{k}} & \left(i_{1} \leq \cdots \leq i_{k}\right) \\ 0 & \text { (otherwise) },\end{cases}
$$

for $1 \leq i_{1}, \ldots, i_{k} \leq N$, where $a_{i}=\#\left\{j \mid i_{j}=i\right\}$ for $1 \leq i \leq N$. It is clear that $\pi_{k} \circ \Delta_{S V}^{(1, \ldots, 1)}=\mathrm{id}$ by the first assertion, and $\Delta_{S V}^{(1, \ldots, 1)}$ is a split injection. The converse is obvious from the first assertion.

Definition 5.5. The graded dual $\left(S^{t} V\right)^{\dagger}$ of $S^{t} V$ is denoted by $D V$ and is called the divided power algebra of $V$. The degree $i$ component of $D V$ is denoted by $D_{i} V$ and is called the $i$-th divided power of $V$.

By definition, $D_{0} V=R$, and $D_{1} V=V$. Moreover, the restriction of $\varphi_{D V}$ to $D_{1} V \otimes$ $D_{1} V=V \otimes V$ is equal to $\beta_{V}$, since ${ }^{t}\left({ }^{t} \beta_{V}\right)=\beta_{V}$. By Proposition 5.2 and Lemma 5.3, we have $\bigwedge V \simeq\left(\bigwedge^{t} V\right)^{\dagger}$ for Jimbo's YB triple of type $A_{N-1}^{(1)}$. By Proposition 5.2 and Lemma 5.4 , we have $S V \simeq D V$ for Jimbo's YB triple when $[n]_{q^{2}}$ is a unit for any $n \geq 1$, but not in general.

Proposition 5.6. Let $V=(V, \beta, \gamma)$ be $a \mathrm{YB}$ triple with finitely generated $V$ and free $S^{t} V$. Then the iterated coproduct $\Delta_{D V}^{\left(1^{k}\right)}: D_{k} V \rightarrow{ }^{t}\left(T_{k}{ }^{t} V\right)=T_{k} V$ is a split injection. The image of this map is $\left(T_{k} V\right)^{\mathfrak{B}_{k}}$.

Proof. The first assertion is clear, since the coproduct of $D V$ is the dual of the multiplication of $S^{t} V$. By the definition of the symmetric algebra, the sequence

$$
\oplus_{1 \leq i \leq k-1} T_{k}{ }^{t} V \stackrel{\sum_{i}\left(1-{ }^{t} \beta_{i}\right)}{\longrightarrow} T_{k}{ }^{t} V \stackrel{p}{\longrightarrow} S_{k}{ }^{t} V \longrightarrow 0
$$

is exact, where $p$ is the projection. Taking the dual, we see that the sequence

$$
\oplus_{1 \leq i \leq k-1} T_{k} V \stackrel{\sum_{i}\left(1-\beta_{i}\right)}{\longleftarrow} T_{k} V \longleftarrow \stackrel{\Delta_{D V}^{\left(1^{k}\right)}}{\longleftarrow} D_{k} V \longleftarrow 0
$$

is also exact. This shows that $D_{k} V$ is the intersection of $\operatorname{Ker}\left(1-\beta_{i}\right)$ for $1 \leq i \leq k-1$. Since $\mathfrak{B}_{k}$ is generated by the $b_{i}$ 's, the second assertion is now clear.

EXAMPLE 5.7. Let $V=(V, \beta, \gamma)$ be Jimbo's YB triple of type $A_{N-1}^{(1)}$. If $[2]_{q^{2}}=0$, then the $R$-submodule $\Pi_{2} V$ of $S_{2} V$ generated by $u_{1}^{2}, \ldots, u_{N}^{2}$ is also an $S E$-comodule, and we have an exact sequence

$$
0 \rightarrow \Pi_{2} V \rightarrow S_{2} V \rightarrow \bigwedge_{2} V \rightarrow 0 .
$$

However $\bigwedge_{2} V$ is not a quotient of $D_{2} V$ (see Section 7). Hence, we have $S_{2} V \neq D_{2} V$ 
as $S E$-comodules. The coproduct $S_{2} V \rightarrow V \otimes V$ vanishes on $\Pi_{2} V$, so the coalgebra structure of $S V$ is different from that of $D V$.

Let $\left(W, \beta_{W}, \gamma_{W}\right)$ be another YB triple such that $S W, \wedge W$ and $S^{t} W$ are free, and $W$ is finite. Consider a map $f \in \mathscr{Y}_{\mathscr{B}_{R}}\left(\left(V, \beta_{V}\right),\left(W, \beta_{W}\right)\right)$. Then, $T f:\left(T V, T\left( \pm \beta_{V}\right)\right) \rightarrow(T W$, $\left.T\left( \pm \beta_{W}\right)\right)$ is a homomorphism of YB algebras. Since $T_{2} T f: T_{2}\left(T V, T\left( \pm \beta_{V}\right)\right) \rightarrow T_{2}(T W$, $\left.T\left( \pm \beta_{W}\right)\right)$ is a homomorphism of algebras, it follows that $T f$ is also a homomorphisms of coalgebras, hence is a homomorphism of YB bialgebras. It is easy to see that $T f$ induces $\bigwedge f:\left(\bigwedge V, \varphi_{\wedge V}\right) \rightarrow\left(\bigwedge W, \varphi_{\bigwedge W}\right), S f:\left(S V, \psi_{S V}\right) \rightarrow\left(S W, \psi_{s W}\right)$ and $D f:(D V$, $\left.\psi_{D V}\right) \rightarrow\left(D W, \psi_{D W}\right)$. The maps $\bigwedge f, S f$ and $D f$ are homomorphisms of YB bialgebras, algebras and coalgebras, respectively. These maps preserve the grading, so we obtain $\bigwedge_{i} f, S_{i} f$ and $D_{i} f$ for $i \geq 0$. If $f$ is also an element of $\mathscr{Y} \mathscr{B}_{R}\left(\left(V, \gamma_{V}\right),\left(W, \gamma_{W}\right)\right)$, then $S f$ and $D f$ are homomorphisms of YB bialgebras.

Let $M$ be a finite free $R$-module. Then $M^{*} \otimes M$ and $M \otimes M^{*}=\left(M^{*}\right)^{*} \otimes M^{*}$ are coalgebras (see Section 3 ). It is easy to see that the trivial twisting $\tau_{M, M^{*}}: M \otimes M^{*} \rightarrow$ $M^{*} \otimes M$ is an anti-homomorphism of coalgebras. Consider an $R$-coalgebra $A$ and a coaction $\omega_{M}: M \rightarrow M \otimes A$. Then, the composite map $\mathrm{cf}_{M^{\circ}} \tau_{M_{M_{M}}}: M \otimes M^{*} \rightarrow A$ is an anti-homomorphism of coalgebras, and yields a left comodule structure of $M^{*}$. The left $A$-comodule $M^{*}$ is called the $R$-dual of the $A$-comodule $M$. For another right $A$-comodule $N$ with finite $R$-module structure, the identification $(M \otimes N)^{*} \simeq M^{*} \otimes N^{*}$ is an isomorphism of left $A$-comodules. Similarly, the $R$-duals of left $A$-comodules are defined, and we have $M^{* *} \simeq M$.

Lemma 5.8. Let $\left(V, \beta_{V}\right) \in \operatorname{ob} \mathscr{Y} \mathscr{B}_{R}$. Then $S \tau_{V, V^{*}}: S\left(V \times V^{\vee}\right) \rightarrow S\left(V^{\vee} \times V\right)$ is an isomorphism of algebras, and is an anti-isomorphism of coalgebras.

Proof. Since $\tau_{V, V^{*}} \in \mathscr{Y} \mathscr{B}_{R}\left(V \times V^{\vee}, V^{\vee} \times V\right)$ is an isomorphism, $S \tau_{V, V^{*}}$ is an isomorphism of algebras. Since $\tau_{V, V^{*}}: V \otimes V^{*} \rightarrow V^{*} \otimes V$ is an anti-homomorphism of coalgebras, and $S\left(V \times V^{\vee}\right)$ is generated by elements of degree one, $S \tau_{V, V^{*}}$ is also an anti-homomorphism of coalgebras.

Note that $E=V \times V^{\vee}$ is the internal End of $V$. Hence, $T V^{*}$ is a right $S E$-comodule, and $\beta^{\vee},{ }^{t} \beta=\left(\beta^{\vee}\right)^{-1}$ are homomorphisms of $S E$-comodules. So $T^{t} V, \bigwedge^{t} V$ and $S^{t} V$ are $S E$-equivariant graded YB bialgebras. With the anti-coalgebra algebra homomorphism $S \tau_{V, V^{*}}$ these algebras are viewed as left $S E^{\vee}$-equivariant graded YB bialgebras. Taking the dual comodules of each degree, the graded duals $\left(T^{t} V\right)^{\dagger},\left(\bigwedge^{t} V\right)^{\dagger}$ and $D V=\left(S^{t} V\right)^{\dagger}$ are (right) $S E$-equivariant graded YB bialgebras. It is easy to see that the natural identification $\left(T^{t} V\right)^{\dagger} \simeq T V$ of graded YB bialgebras is also an $S E^{\vee}$-comodule isomorphism. Hence, $D_{k} V$ is a subcomodule of $V^{\otimes k}$ via the injection $\Delta: D_{k} V \rightarrow V^{\otimes k}$. Since $\Phi^{\wedge}: \bigwedge V \rightarrow\left(\bigwedge^{t} V\right)^{\dagger}$ and $\Phi^{S V}: S V \rightarrow D V$ are induced by $T V \simeq\left(T^{t} V\right)^{\dagger}$ (see Proposition 5.2), these maps are $S E^{\vee}$-equivariant.

ReMARK 5.9. Since $\beta^{-1}$ is an $S E^{\vee}$-comodule map, $T V^{-1}, \bigwedge V^{-1}$ and $S V^{-1}$ have 
$S E$-comodule structures. Clearly, the identification $T V \simeq T V^{-1}$ is an isomorphism of both algebras and $S E^{\vee}$-comodules. Let us compare $\bigwedge V$ and $\bigwedge V^{-1}$. They are the quotient algebras of $T V=T V^{-1}$ defined by the same ideal $(\operatorname{Ker}(1-\beta))=\left(\operatorname{Ker}\left(1-\beta^{-1}\right)\right)$. Hence, $(\bigwedge V, \varphi \wedge)$ and $\left(\bigwedge V^{-1}, \varphi_{\bigwedge^{-1} V^{-1}}\right)$ are isomorphic as $S E^{\vee}$-equivariant YB algebras. For each $k \geq 1$, the image of the coproduct maps $\operatorname{Im} \Delta_{\Lambda}^{\left(1^{k}\right)}=\operatorname{Im}\left(\sum_{\sigma \in \Theta_{k}}(-\beta)(\sigma)\right)$ and $\operatorname{Im} \Delta_{\wedge V^{-1}}^{\left(1^{k}\right)}=\operatorname{Im}\left(\sum_{\sigma \in \Theta_{k}}\left(-\beta^{-1}\right)(\sigma)\right)$ agree. It follows that $\left(\bigwedge V, \varphi_{\wedge V}\right)$ and $\left(\bigwedge V^{-1}\right.$, $\left.\varphi \wedge^{-1} V^{-1}\right)$ are also isomorphic as $S E^{\vee}$-equivariant YB coalgebras. But they are not isomorphic as YB bialgebras in general. To illustrate this, consider Jimbo's YB triple $(V, \beta, \gamma)$ of type $A_{N-1}^{(1)}$, and compare the maps

$$
\bigwedge_{2} V \stackrel{\Delta}{\longrightarrow} V \otimes V \stackrel{m}{\longrightarrow} \bigwedge_{2} V \text { and } \bigwedge_{2} V^{-1} \stackrel{\Delta}{\longrightarrow} V^{-1} \otimes V^{-1} \stackrel{m}{\longrightarrow} \bigwedge_{2} V^{-1} .
$$

The first is the multiplication by $1+q^{2}$, while the second is multiplication by $1+q^{-2}$. Similar facts on symmetric algebras and divided power algebra should be noted.

Proposition 5.10. Let $V=\left(V, \beta_{V}, \gamma_{V}\right)$ be Jimbo's YB triple of type $A_{N-1}^{(1)}$ and $E=\left(E, \beta_{E}, \gamma_{E}\right)$ be as in $\S 4$ Example (2). Then we have:

1. For any $k \geq 0$, the comultiplication $\Delta_{\Lambda}^{\left(1^{k}\right)}$ is a split injection. Hence, we have $\bigwedge E \simeq\left(\bigwedge^{t} E\right)^{\dagger}$ as graded $\mathrm{YB}$-bialgebras.

2. $\Delta_{S E}^{\left(1^{k}\right)}: S_{k} E \rightarrow T_{k} E$ is injective if $R=Z\left[Q, Q^{-1}\right]$, where $Q$ is an indeterminate.

Proof. 1. We define a map $\rho_{k}: E^{\otimes k} \rightarrow \bigwedge_{k} E$ by

$$
\rho_{k}\left(x_{i_{1} j_{1}} \otimes \cdots \otimes x_{i_{k} j_{k}}\right)= \begin{cases}x_{i_{1} j_{1}} \wedge \cdots \wedge x_{i_{k} j_{k}} & \left({ }^{\forall} l i_{l}<i_{l+1}, \text { or } j_{l}>j_{l+1} \text { and } i_{l}=i_{l+1}\right) \\ 0 & \text { (otherwise) } .\end{cases}
$$

Then $\rho_{k} \circ \Delta_{\wedge E}^{\left(1^{k}\right)}=\mathrm{id} \bigwedge_{\wedge_{k} E}$. Hence, $\Delta_{\bigwedge}^{\left(1^{k}\right)}$ splits. The second assertion is a consequence of Proposition 5.2. by

2. We may localize, and assume that $R=Q(Q)$. We define a map $\pi_{k}: E^{\otimes k} \rightarrow S_{k} E$

$$
\pi_{k}\left(x_{i_{1} j_{1}} \otimes \cdots \otimes x_{i_{k} j_{k}}\right)=\left\{\begin{aligned}
\left({ }_{l=1}^{N} \prod_{l^{\prime}}{ }^{N}=1\right. & {\left.\left[u_{l, l^{\prime}}\right]_{Q^{-2}}^{!}\right)^{-1} \cdot x_{i_{1} j_{1}} \cdots x_{i_{k} j_{k}} } \\
& \text { (if } \left.{ }^{\forall} h i_{h}<i_{h+1}, \text { or } i_{h}=i_{h+1} \text { and } j_{h}<j_{h+1}\right) \\
0 & \text { (otherwise), }
\end{aligned}\right.
$$

where $\left.u_{l, l^{\prime}}=\#\left\{h \mid\left(i_{h}, j_{h}\right)=\left(l, l^{\prime}\right)\right\}\right)$. Then $\pi_{k} \circ \Delta_{S E}^{\left(1^{k}\right)}=\mathrm{id}_{S_{k} E}$. Hence $\Delta_{S E}^{\left(1^{k}\right)}$ is an injection.

Assume that $R$ is a field. Since $\left(S_{2} V\right)^{*}=(\operatorname{Coker}(1-\beta))^{*}$ is identified with $D_{2}{ }^{t} V=$ $\operatorname{Ker}\left(1-{ }^{t} \beta\right)$, the Koszul complex of the quadratic algebra $S V$ defined in [26] is nothing but $S V \otimes\left(\bigwedge^{t} V\right)^{\dagger}$. The boundary map is the composite map

$$
\partial: S V \otimes\left(\bigwedge^{t} V\right)^{\dagger} \stackrel{1 \otimes \Delta}{\longrightarrow} S V \otimes V \otimes\left(\bigwedge^{t} V\right)^{\dagger} \stackrel{m \otimes 1}{\longrightarrow} S V \otimes\left(\bigwedge^{t} V\right)^{\dagger},
$$


where $\Delta$ is the abbreviation for the map

$$
\left(\bigwedge^{t} V\right)^{\dagger} \stackrel{\Delta}{\longrightarrow}\left(\bigwedge^{t} V\right)^{\dagger} \otimes\left(\bigwedge^{t} V\right)^{\dagger} \stackrel{\operatorname{proj} \otimes 1}{\longrightarrow}\left(\bigwedge_{1}{ }^{t} V\right)^{*} \otimes\left(\bigwedge^{t} V\right)^{\dagger}=V \otimes\left(\bigwedge^{t} V\right)^{\dagger},
$$

and $m$ is the product in $S V$. If $S V$ is a (homogeneous) Koszul algebra (see [28]), then $S V \otimes\left(\bigwedge^{t} V\right)^{\dagger}$ is a resolution of the left $S V$-module $R=S V /(V)$. If $V=(V, \beta, \gamma)$ is Jimbo's YB triple of type $A_{N-1}^{(1)}$, then $S V \otimes\left(\bigwedge^{t} V\right)^{\dagger}$ is rewritten as $S V \otimes \wedge V$, and the boundary map is described explicitly as

$$
\begin{array}{r}
\partial\left(a \otimes u_{i_{1}} \wedge \cdots \wedge u_{i_{k}}\right)=\sum_{j=1}^{k}(-q)^{j-1} a u_{i_{j}} \otimes u_{i_{1}} \wedge \cdots \wedge \check{u}_{i_{j}} \wedge \cdots \wedge u_{i_{k}} \\
\left(1 \leq i_{1}<\cdots<i_{k} \leq N\right) .
\end{array}
$$

In this case, $S V$ is a Koszul algebra [32, Theorem 5.3], and the Koszul complex $S V \otimes \wedge V$ is acyclic.

REMARK 5.11. Let $V$ be a YB pair. By the discussion in the proof of Proposition 5.6, the dual of the projection $T_{k} V \rightarrow S_{k} V$ is naturally identified with the inclusion $\left(T_{k} V^{\vee}\right)^{\mathfrak{B}_{k}} \rightarrow T_{k} V^{\vee}$.

6. Schur modules and Weyl modules. Throughout this section, $\left(V, \beta_{V}\right)$ is Jimbo's YB pair of type $A_{N-1}^{(1)}$ (cf. (1.2)) over the base ring $R$ determined by a unit $q \in R$.

For (infinite) sequences of integers $\lambda=\left(\lambda_{1}, \lambda_{2}, \ldots\right)$ and $\mu=\left(\mu_{1}, \mu_{2}, \ldots\right)$, we define the sum $\lambda+\mu$ to be the sequence $\left(\lambda_{1}+\mu_{1}, \lambda_{2}+\mu_{2}, \ldots\right)$, and we define $k \cdot \lambda$ to be the sequence $\left(k \cdot \lambda_{1}, k \cdot \lambda_{2}, \ldots\right)$ for $k \in Z$. We also denote $\operatorname{supp}(\lambda)=\left\{i \in N \mid \lambda_{i} \neq 0\right\}$. We will denote by $\Omega^{+}$the set $\left\{\lambda\right.$ : a sequence of integers $\left.\right|^{\forall} i \in N, \lambda_{i} \geq 0$ and $\left.\#(\operatorname{supp}(\lambda))<\infty\right\}$. For $\lambda \in \Omega^{+}$, we define the length of $\lambda$ by $\ell(\lambda)=\max \operatorname{supp}(\lambda)$, and the degree of $\lambda$ by $|\lambda|=$ $\sum_{i \in N} \lambda_{i}$. When $\lambda=(0,0, \ldots)$, we define $\ell(\lambda)=0$. We set $\Omega^{-}=\left\{\lambda \in \Omega^{+} \mid{ }^{\forall} i \in N \lambda_{i} \geq \lambda_{i+1}\right\}$ and call an element of $\Omega^{-}$a partition. For any $\lambda \in \Omega^{+}$, its transpose $\tilde{\lambda}$ is defined to be the partition which satisfies $\tilde{\lambda}_{i}=\#\left\{j \in N \mid \lambda_{j} \geq i\right\}$ for any $i \in N$. It is easy to see that $(\tilde{\lambda})^{\sim}$ is the partition obtained by rearrangement of the sequence $\lambda$ in non-increasing order.

We introduce a lexicographic order $<$ into $\Omega^{+}$. Namely, for two elements $\lambda$ and $\mu \in \Omega^{+}$, we say that $\lambda>\mu$ when there exists some $j$ such that $\lambda_{j}>\mu_{j}$ and $\lambda_{i}=\mu_{i}$ for any $i<j$. With this ordering, $\Omega^{+}$is a totally ordered set.

We will denote the partition $(0,0, \ldots)$ of degree zero by 0 .

For $\lambda, \mu \in \Omega^{+}$, we say that $\lambda \supset \mu$ if and only if $\lambda_{i} \geq \mu_{i}$ for each $i \in N$. It is clear that $\supset$ is an order and $\lambda \supset \mu$ implies $\lambda \geq \mu$. It holds that $\lambda \supset \mu$ if and only if $\tilde{\lambda} \supset \tilde{\mu}$. For $\lambda$, $\mu \in \Omega^{-}$, we say that $\lambda / \mu$ is a skew partition, if $\lambda \supset \mu$.

Definition 6.1. The diagram (or shape) $\Delta_{\lambda}$ of an element $\lambda \in \Omega^{+}$is the set $\left\{(i, j) \in N^{2} \mid j \leq \lambda_{i}\right\}$. We use the convention as in the case of matrices, namely, the row index $i$ increases as one goes downward, and the column index $j$ increases from left to right (see Figure). The skew shape of a pair $\lambda, \mu \in \Omega^{+}$with $\lambda \supset \mu$ is $\Delta_{\lambda / \mu}:=\Delta_{\lambda} \backslash \Delta_{\mu}$. 


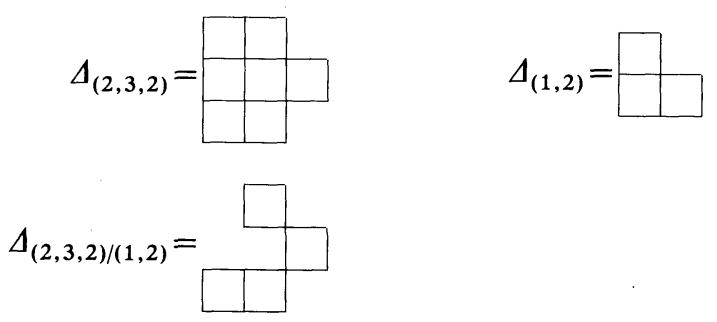

FIGURE.

Clearly, $\Delta_{\lambda / 0}=\Delta_{\lambda}$. So we will always adapt the terminology for skew-shape (or skew partition) to the terminology for shape (or partition) by letting $\mu=0$.

Definition 6.2. Let $\boldsymbol{X}$ be a set and let $\lambda, \mu \in \Omega^{+}$. We define $\operatorname{Tab}_{\lambda / \mu}(\boldsymbol{X})$ to be the set $\operatorname{Map}\left(\Delta_{\lambda / \mu}, X\right)$. An element of $\operatorname{Tab}_{\lambda / \mu}(X)$ is called a tableau of shape $\lambda / \mu$ with values in the set $X$. For $S \in \operatorname{Tab}_{\lambda / \mu}(X)$, we say that $S$ is a Young tableau, if $X$ is the set $[1,|\lambda|-|\mu|]$ and $S$ is a bijection.

Pictorially, a tableau $S \in \operatorname{Tab}_{\lambda / \mu} X$ is expressed as an array of elements in $X$ in which $S(i, j)$ occupies the intersection of the $i$-th row and the $j$-th column. For example, if $\lambda=(4,3,3), \mu=(2,1)$ and

$$
\begin{aligned}
& 63 \\
& S=14 \text {, } \\
& 527
\end{aligned}
$$

then $S$ is a Young tableau, and $S(3,2)=2$.

Definition 6.3. Let $\boldsymbol{Y}$ be a totally ordered set. A tableau $S \in \operatorname{Tab}_{\lambda / \mu}(\boldsymbol{Y})$ is said to be row-standard if the rows of $S$ are strictly increasing, i.e., if we have $S\left(i, \mu_{i}+1\right)<$ $S\left(i, \mu_{i}+2\right)<\cdots<S\left(i, \lambda_{i}\right)$ for any $i$. The tableau $S$ is said to be column-standard if the columns of $S$ are non-decreasing, i.e., if for all $1 \leq j \leq \lambda_{1}$ we have $S(i, j) \leq S(i+1, j)$ whenever $(i, j)$ and $(i+1, j)$ are both in $\Delta_{\lambda / \mu} . S$ is said to be standard if it is both row- and column-standard. We denote by $\operatorname{Row}_{\lambda / \mu} \boldsymbol{Y}\left(\operatorname{resp} . \mathrm{St}_{\lambda / \mu} \boldsymbol{Y}\right.$ ) the subset of $\operatorname{Tab}_{\lambda / \mu} \boldsymbol{Y}$ consisting of all row-standard (resp. standard tableaux).

Definition 6.4. A tableau $T \in \operatorname{Tab}_{\lambda / \mu}(Y)$ is said to be co-row-standard if the rows of $T$ are non-decreasing, and co-column-standard if the columns of $T$ are strictly increasing. $T$ is said to be co-standard if it is co-row- and co-column-standard. We denote by $\operatorname{Corow}_{\lambda / \mu} \boldsymbol{Y}$ (resp. Cost $\operatorname{Cos}_{\lambda / \mu} \boldsymbol{Y}$ ) the subset of $\mathrm{Tab}_{\lambda / \mu} \boldsymbol{Y}$ consisting of all co-rowstandard (resp. co-standard) tableaux.

Let $\lambda, \mu \in \Omega^{+}$with $\lambda \supset \mu$. We define an element $\chi_{\lambda / \mu}$ in $\Im_{|\lambda|-|\mu|}$ as follows. First we introduce a lexicographic order into the set $\Delta_{\lambda / \mu}$. We define $(i, j)<_{r}\left(i^{\prime}, j^{\prime}\right)$ if $i<i^{\prime}$, or $i=i^{\prime}$ and $j<j^{\prime}$. Since $<_{r}$ is a total ordering, we have a unique order-preserving bijection 
$\alpha_{r, \lambda / \mu}:[1,|\lambda|-|\mu|] \rightarrow\left(\Delta_{\lambda / \mu},<_{r}\right)$. Secondly, we introduce another lexicographic order into $\Delta_{\lambda / \mu}$. We define $(i, j)<_{c}\left(i^{\prime}, j^{\prime}\right)$ if $j<j^{\prime}$, or $j=j^{\prime}$ and $i<i^{\prime}$. We also have a unique orderpreserving bijection $\alpha_{c, \lambda / \mu}:[1,|\lambda|-|\mu|] \rightarrow\left(\Delta_{\lambda / \mu},<_{c}\right)$. We define $\chi_{\lambda / \mu}:=\alpha_{c, \lambda / \mu}^{-1} \circ \alpha_{r, \lambda / \mu}$. Here we show an example.

EXAMPLE 6.5. Let $\lambda=(5,4,2)$ and $\mu=(1,1)$. The permutation $\chi_{\lambda / \mu}$ is described as follows:

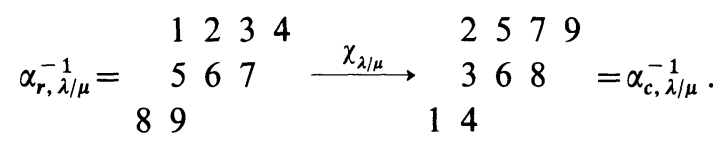

The permutation $w_{i}$ in Proposition 4.2 is nothing but $\chi_{(i, i)}$.

Definition 6.6. For any $\lambda, \mu \in \Omega^{+}$, we define

$$
\begin{aligned}
& \bigwedge_{\lambda / \mu} V=\bigwedge_{\lambda_{1}-\mu_{1}} V \otimes \cdots \otimes \bigwedge_{\lambda_{s}-\mu_{s}} V, \\
& S_{\lambda / \mu} V=S_{\lambda_{1}-\mu_{1}} V \otimes \cdots \otimes S_{\lambda_{s}-\mu_{s}} V, \\
& D_{\lambda / \mu} V=D_{\lambda_{1}-\mu_{1}} V \otimes \cdots \otimes D_{\lambda_{s}-\mu_{s}} V, \\
& T_{\lambda / \mu} V=T_{\lambda_{1}-\mu_{1}} V \otimes \cdots \otimes T_{\lambda_{s}-\mu_{s}} V,
\end{aligned}
$$

when $\lambda \supset \mu$, where $s$ is a sufficiently large integer. If $\lambda \not \mu$, they are all 0 by definition. Note that $T_{\lambda / \mu} V$ is naturally isomorphic to $T_{|\lambda|-|\mu|} V$.

Definition 6.7. Let $\lambda / \mu$ be a skew partition with $\ell(\lambda / \mu)=s$ and $\lambda_{1}=t$. We denote the composite map

$$
\begin{aligned}
& \bigwedge_{\lambda / \mu} V=\bigwedge_{\lambda_{1}-\mu_{1}} V \otimes \cdots \otimes \bigwedge_{\lambda_{t}-\mu_{t}} V \\
& \downarrow \Delta_{\Lambda V}^{\left(1^{\left.\lambda_{1}-\mu_{1}\right)} \otimes \cdots \otimes \Delta_{\Lambda V}^{\left(1^{\lambda_{t}}-\mu_{t}\right)}\right.} \\
& T_{\lambda / \mu} V=T_{\lambda_{1}-\mu_{1}} V \otimes \cdots \otimes T_{\lambda_{t}-\mu_{t}} V=T_{|\lambda|-|\mu|} V \\
& \downarrow\left(q^{-2} \beta\right)\left(\chi_{\lambda / \mu}\right) \\
& T_{\tilde{\lambda} / \tilde{\mu}} V=T_{\tilde{\lambda}_{1}-\tilde{\mu}_{1}} V \otimes \cdots \otimes T_{\tilde{\lambda}_{s}-\tilde{\mu}_{s}} V=T_{|\lambda|-|\mu|} V \\
& \downarrow p \otimes \cdots \otimes p \\
& S_{\tilde{\lambda} / \tilde{\mu}} V=S_{\tilde{\lambda}_{1}-\tilde{\mu}_{1}} V \otimes \cdots \otimes S_{\tilde{\lambda}_{s}-\tilde{\mu}_{s}} V
\end{aligned}
$$

from $\bigwedge_{\lambda / \mu} V$ to $S_{\tilde{\lambda} / \tilde{\mu}} V$ by $d_{\lambda / \mu}(V)$, and call it the $S c h u r$ map (cf. [4]). The image $\operatorname{Im} d_{\lambda / \mu}(V)$ of the Schur map is denoted by $L_{\lambda / \mu} V$, and is called the Schur module of $V$ with respect to the skew partition $\lambda / \mu$.

By definition, $d_{\lambda / \mu}(V)$ and $L_{\lambda / \mu} V$ are the usual Schur map and the Schur module, respectively, when $q=1$. Note that $\left(q^{-2} \beta\right)\left(\chi_{\lambda / \mu}\right)$ in the definition can be replaced by $\beta\left(\chi_{\lambda / \mu}\right),(-\beta)\left(\chi_{\lambda / \mu}\right)$, or $\left(-q^{-2} \beta\right)\left(\chi_{\lambda / \mu}\right)$ for the purpose of defining $L_{\lambda / \mu} V$, since $q$ is a unit 
(using $\beta\left(\chi_{\lambda / \mu}\right)$ might be the simplest; however we use $\left(q^{-2} \beta\right)\left(\chi_{\lambda / \mu}\right)$, since it is more consistent with the notation in [14] (see $\S 8)$ ). It is easy to see that $d_{\lambda / \mu} V$ is an $S E^{\vee}$ homomorphism, and $L_{\lambda / \mu} V$ is an $S E^{\vee}$-comodule. We denote $d_{\lambda / 0}(V)$ and $L_{\lambda / 0} V$ by $d_{\lambda}(V)$ and $L_{\lambda} V$, respectively.

We set $Y(\sigma)=\left\{u_{\sigma 1}<\cdots<u_{\sigma N}\right\}$, the ordered basis of $V$ for $\sigma \in \mathfrak{S}_{N}$, and $X=$ $\left\{u_{1}, \ldots, u_{N}\right\}$ its underlying set.

DeFINITION 6.8. Let $\lambda / \mu$ be a skew partition with $\ell=\ell(\lambda / \mu)$ and $\tilde{\ell}=\ell(\tilde{\lambda} / \tilde{\mu})$, and $S \in \mathrm{Tab}_{\lambda / \mu} X$. We define

$$
\begin{aligned}
& \xi_{S}=S\left(1, \mu_{1}+1\right) \wedge \cdots \wedge S\left(1, \lambda_{1}\right) \otimes \cdots \otimes S\left(\ell, \mu_{\ell}+1\right) \wedge \cdots \wedge S\left(\ell, \lambda_{\ell}\right) \in \wedge_{\lambda / \mu} V, \\
& \eta_{S}=S\left(\tilde{\mu}_{1}+1,1\right) \cdots S\left(\tilde{\lambda}_{1}, 1\right) \otimes \cdots \otimes S\left(\tilde{\mu}_{\tilde{\ell}}+1, \tilde{\ell}\right) \cdots S\left(\tilde{\lambda}_{\tilde{\ell}}, \tilde{\ell}\right) \in S_{\tilde{\lambda} / \tilde{\mu}} V, \\
& \zeta_{S}=S\left(1, \mu_{1}+1\right) \otimes \cdots \otimes S\left(1, \lambda_{1}\right) \otimes \cdots \otimes S\left(\ell, \mu_{\ell}+1\right) \otimes \cdots \otimes S\left(\ell, \lambda_{\ell}\right) \in T_{\lambda / \mu} V, \\
& \tilde{\zeta}_{S}=S\left(\tilde{\mu}_{1}+1,1\right) \otimes \cdots \otimes S\left(\tilde{\lambda}_{1}, 1\right) \otimes \cdots \otimes S\left(\tilde{\mu}_{\tilde{\ell}}+1, \tilde{\ell}\right) \otimes \cdots \otimes S\left(\tilde{\lambda}_{\tilde{\ell}}, \tilde{\ell}\right) \in T_{\tilde{\lambda} / \tilde{\mu}} V .
\end{aligned}
$$

For $\sigma \in \mathfrak{S}_{N}$, we set

$$
\begin{aligned}
\bigwedge_{\lambda / \mu} Y(\sigma) & =\left\{\xi_{S} \mid S \in \operatorname{Row}_{\lambda / \mu} Y(\sigma)\right\}, \\
L_{\lambda / \mu} Y(\sigma) & =\left\{d_{\lambda / \mu}(V)\left(\xi_{S}\right) \mid S \in \operatorname{St}_{\lambda / \mu} Y(\sigma)\right\} .
\end{aligned}
$$

It is obvious that $\bigwedge_{\lambda / \mu} Y(\sigma)$ and the set $\left\{\eta_{S} \mid S \in \operatorname{Tab}_{\lambda / \mu} Y(\sigma), S\right.$ is column-standard $\}$ are bases of $\bigwedge_{\lambda / \mu} V$ and $S_{\tilde{\lambda} / \tilde{\mu}} V$, respectively, for any $\sigma \in \Xi_{N}$. It follows that $\widetilde{\zeta}_{S}=\tau\left(\chi_{\lambda / \mu}\right) \cdot \zeta_{S}$, where $\tau$ is the trivial twisting. In the rest of this section, we sometimes tacitly identify $T_{\lambda / \mu} V$ with $T_{|\lambda|-|\mu|} V$. In such an occasion, we also identify $S \in \operatorname{Tab}_{\lambda / \mu} X$ with $S \circ \alpha_{r, \lambda / \mu} \in \operatorname{Map}([1, N], X)$. For an arbitrary totally ordered set $\boldsymbol{Y}$ and skew partition $\lambda / \mu$, we introduce two (pseudo-) orders into $\operatorname{Tab}_{\lambda / \mu} Y$, as follows:

DEFINITION 6.9. For a skew partition $\lambda / \mu, I \subset N$, totally ordered set $\boldsymbol{Y}, \boldsymbol{Y}_{0} \subset \boldsymbol{Y}$ and $S \in \operatorname{Tab}_{\lambda / \mu} Y$, we define

$$
n_{I}\left(S, \boldsymbol{Y}_{0}\right)=\#\left\{(i, j) \in \Delta_{\lambda / \mu} \mid i \in I, \quad S(i, j) \in \boldsymbol{Y}_{0}\right\} .
$$

Definition 6.10. Let $S$ and $S^{\prime}$ be elements in the set $\operatorname{Tab}_{\lambda / \mu} Y$. We say that $S \leq S^{\prime}$ when it holds

$$
n_{\{1, \ldots, i\}}(S,(-\infty, y]) \geq n_{\{1, \ldots, i\}}\left(S^{\prime},(-\infty, y]\right)
$$

for any $i \in[1, \ell(\lambda / \mu)]$ and $y \in \boldsymbol{Y}$, where $(-\infty, y]$ denotes the subset $\{z \in \boldsymbol{Y} \mid z \leq y\}$ of $Y$. We say that $S \leq_{\text {lex }} S^{\prime}$ when $S=S^{\prime}$ or $S \circ \alpha_{r, \lambda / \mu}(j)<S^{\prime} \circ \alpha_{r, \lambda / \mu}(j)$, where $j$ is the minimum of the numbers $i$ such that $S \circ \alpha_{r, \lambda / \mu}(i) \neq S^{\prime} \circ \alpha_{r, \lambda / \mu}(i)$. We say that $S<S^{\prime}$ (resp. $S<_{\text {lex }} S^{\prime}$ ) when $S \leq S^{\prime}$ and $S \ngtr S^{\prime}$ (resp. $S \leq_{\text {lex }} S^{\prime}$ and $S \neq S^{\prime}$ ).

Note that $\leq$ is a pseudo-order for $\operatorname{Tab}_{\lambda / \mu} \boldsymbol{Y}$, and $\leq_{\text {lex }}$ is a total order of $\operatorname{Tab}_{\lambda / \mu} \boldsymbol{Y}$. The restriction of $\leq$ to $\operatorname{Row}_{\lambda / \mu} Y$ is an order, and $S, S^{\prime} \in \operatorname{Row}_{\lambda / \mu} Y, S \leq S^{\prime}$ imply $S \leq_{\text {lex }} S^{\prime}$. 
EXAMPLE 6.11. Let $\lambda=(3,2), \mu=(1), Y=\{1<2<3\}$, and $S_{1}, S_{2}$ and $S_{3}$ be as follows:

$$
S_{1}=\begin{array}{ll}
1 & 3 \\
2 & 2
\end{array}, \quad S_{1}=\begin{array}{ll}
2 & 2 \\
1 & 3
\end{array}, \quad S_{3}=\begin{array}{ll}
3 & 1 \\
2 & 2
\end{array} .
$$

Then we have $S_{1} \leq S_{3} \leq S_{1}$ since $S_{3}$ is obtained by row-permutation on the entries of $S_{1}$. We have $S_{2} \nless S_{1} \nless S_{2} \nless S_{3} \nless S_{2}$, since $n_{\{1\}}\left(S_{1},\{1\}\right)=n_{\{1\}}\left(S_{3},\{1\}\right)=1>0=n_{\{1\}}\left(S_{2},\{1\}\right)$ and $n_{\{1\}}\left(S_{1},\{1,2\}\right)=n_{\{1\}}\left(S_{3},\{1,2\}\right)=1<2=n_{\{1\}}\left(S_{2},\{1,2\}\right)$. Since $S_{1}(1,2)=1<S_{2}(1,2)$ $=2<S_{3}(1,2)=3$, we have $S_{1}<_{\text {lex }} S_{2}<_{\text {lex }} S_{3}$.

Returning to the situation $X=\left\{u_{1}, \ldots, u_{N}\right\}$, we denote $S \leq_{\sigma} S^{\prime}$ (resp. $S \leq_{\text {lex }, \sigma} S^{\prime}$ ) for $S, S^{\prime} \in \operatorname{Tab}_{\lambda / \mu} X$, when $S \leq S^{\prime}$ (resp. $S \leq_{\text {lex }} S^{\prime}$ ) with $S, S^{\prime}$ viewed as elements of $\operatorname{Tab}_{\lambda / \mu} Y(\sigma)$. We will omit the subscript $\sigma$ if $\sigma=$ id. Hence, $S \leq S^{\prime}$ (resp. $S \leq_{\text {lex }} S^{\prime}$ ) and $S \geq_{\omega_{0}} S^{\prime}$ (resp. $S \geq_{\text {lex, } \omega_{0}} S^{\prime}$ ) are equivalent, where $\omega_{0} \in \Im_{N}$ is the unique element with maximal length.

For $k \in N$ and $\rho \in \Im_{k}$, we define the decomposition of $\rho$ by induction on $k$. We let $\rho_{1}=\left(\begin{array}{lll}1 & 2 \cdots \rho^{-1}\end{array}\right)$, the cyclic permutation. Since $\rho \rho_{1}^{-1}(1)=1$, we may assume that $\rho \rho_{1}^{-1} \in \mathfrak{S}_{k-1}$. Let $\rho \rho_{1}^{-1}=\rho_{k-1} \cdots \rho_{2}$ be the decomposition of $\rho \rho_{1}^{-1}$. We define the decomposition of $\rho$ to be $\rho=\rho_{k-1} \cdots \rho_{1}$. Note that this decomposition is always a reduced expression.

Lemma 6.12. Let $\lambda / \mu$ be a skew partition, and $S \in \operatorname{Tab}_{\lambda / \mu} Y$. Then we have

$$
\beta\left(\chi_{\lambda / \mu}\right) \zeta_{S}=q^{*} \tilde{\zeta}_{S}+\sum_{S^{\prime}>S} c_{S^{\prime}} \tilde{\zeta}_{S^{\prime}} \quad\left(c_{S^{\prime}} \in Z\left[q, q^{-1}\right]\right),
$$

where by $q^{*}$ we mean some power of $q$.

ProOF. We set $k=|\lambda|-|\mu|$, and proceed by induction on $k$. We denote by $\mu^{\prime}$ the unique partition such that $\Delta_{\lambda / \mu^{\prime}}$ is obtained by removal of the box $\alpha_{c, \lambda / \mu}(1)$ from $\Delta_{\lambda / \mu}$. We denote $\chi_{\lambda / \mu}^{-1}(1)$ by $d$ (in Example $6.5, \alpha_{c, \lambda / \mu}(1)=(3,1)$ and $d=8$ ). If the decomposition of $\chi_{\lambda / \mu}$ is $\chi_{\lambda / \mu}=\rho_{k-1} \cdots \rho_{2} \rho_{1}$, then we have $\rho_{k-1} \cdots \rho_{2}=\chi_{\lambda / \mu}$, with the natural identification of $\mathfrak{S}_{k-1}$ with the isotropy group of 1 in $\mathfrak{S}_{k}$. Hence,

$$
\tau\left(\chi_{\lambda / \mu}^{-1}\right) \beta\left(\chi_{\lambda / \mu}\right)=\left[1_{V} \otimes \tau\left(\chi_{\lambda / \mu^{\prime}}^{-1}\right) \beta\left(\chi_{\lambda / \mu^{\prime}}\right)\right] \circ\left[\tau\left(\rho_{1}^{-1}\right) \beta\left(\rho_{1}\right)\right],
$$

where we identify $T_{\lambda / \mu} V$ with $V \otimes T_{\lambda / \mu^{\prime}} V$ via the isomorphism $\tau\left(\rho_{1}\right)$. By the remark after Definition 6.8, it suffices to show that we have an expression

$$
\tau\left(\chi_{\lambda / \mu}^{-1}\right) \beta\left(\chi_{\lambda / \mu}\right) \zeta_{S}=q^{*} \zeta_{S}+\sum_{S^{\prime}>S} c_{S^{\prime}} \zeta_{S^{\prime}}
$$

By the induction hypothesis, it also suffices to prove that we have an expression

$$
\tau\left(\rho_{1}^{-1}\right) \beta\left(\rho_{1}\right) \zeta_{S}=q^{*} \zeta_{S}+\sum_{S^{\prime}>S} c_{S^{\prime}} \zeta_{S^{\prime}}
$$


We set $\pi_{i}=\sigma_{i} \cdots \sigma_{d-1}$ for $1 \leq i \leq d-1$, where $\sigma_{j}$ is the transposition $(j j+1)$. Note that $\rho_{1}=\pi_{1}$. To prove that we have an expression (*), we will show that

$$
\beta\left(\pi_{i}\right) \zeta_{S}=\sum_{I} c_{I}^{i} \zeta_{S_{i}(I)} \quad\left(c_{I}^{i} \in Z\left[q, q^{-1}\right], \quad c_{\varnothing}^{i}=q^{*}\right)
$$

for any $i \in[1, d-1]$, where the sum is taken over all subsequences $I=\left(i_{1}, i_{2}, \ldots, i_{h}\right)$ of $(i, i+1, \ldots, d-1)$ such that $S\left(i_{1}\right)<S\left(i_{2}\right)<\cdots<S\left(i_{h}\right)<S(d)$. The empty subsequence is denoted by $\varnothing$. For such a subsequence $I$, let $J_{i}=\left(j_{1}, \ldots, j_{d-h-i}\right)$ be the complementary subsequence of $I$ in $(i, i+1, \ldots, d-1)$. The tableau $S_{i}^{\prime}(I)$ is defined by $S_{i}^{\prime}(I)=\sigma_{j_{1}} \cdots$ $\sigma_{j_{d-h-i}} \cdot S\left(=S \circ \sigma_{j_{d-h-i}} \cdots \sigma_{j_{1}}\right)$. We shall prove (**) by descending induction on $i$. For each subsequence $I=\left(i_{1}, \ldots, i_{h}\right)$ of $(i+1, \ldots, d-1)$, we have

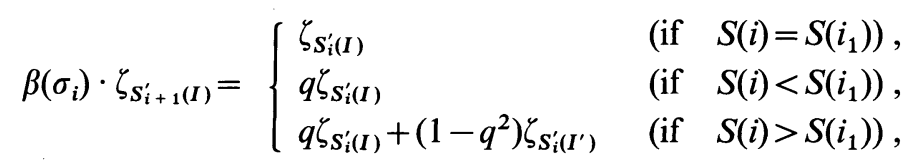

by (1.3), where we assume that $i_{1}=d$ when $I$ is empty, and $I^{\prime}$ is the sequence $\left(i, i_{1}, \ldots, i_{h}\right)$. So $(* *)$ holds by the induction hypothesis. Moreover, we have

$$
\pi_{i}^{-1} \cdot S_{i}^{\prime}(I)=\pi_{i+1}^{-1} \sigma_{i} S_{i}^{\prime}(I)=\pi_{i+1}^{-1} S_{i+1}^{\prime}(I)
$$

and

$$
\pi_{i}^{-1} \cdot S_{i}^{\prime}\left(I^{\prime}\right)=\pi_{i+1}^{-1} \sigma_{i} \pi_{i+1} \pi_{i+1}^{-1} S_{i}^{\prime}\left(I^{\prime}\right)=(i d) \pi_{i+1}^{-1} S_{i+1}^{\prime}(I) .
$$

Since $\left(\pi_{i+1}^{-1} \cdot S_{i+1}^{\prime}(I)\right)(i)=S(i)>S\left(i_{1}\right)=\left(\pi_{i+1}^{-1} S_{i+1}^{\prime}(I)\right)(d)$ and $\alpha_{r, \lambda / \mu}(i)$ lies in an earlier row than the row in which $\alpha_{r, \lambda / \mu}(d)$ lies, it is easy to see that $\pi_{i}^{-1} S_{i}^{\prime}\left(I^{\prime}\right)<\pi_{i+1}^{-1} S_{i+1}^{\prime}$ (I) (cf. [4, Lemma II. 2. 14]).

Using the descending induction again, we have $\rho_{1}^{-1} \cdot S_{1}^{\prime}(\varnothing)=S>\rho_{1}^{-1} S_{1}^{\prime}(I)$ for any non-empty $I$. By $(* *)$, we have an expression

$$
\tau\left(\rho_{1}^{-1}\right) \beta\left(\rho_{1}\right) \zeta_{S}=\sum_{I} c_{I}^{1} \zeta_{\rho_{1}^{-1}} \cdot s_{1}^{\prime}(I) \quad\left(c_{I}^{1} \in Z\left[q, q^{-1}\right], \quad c_{\varnothing}^{1}=q^{*}\right)
$$

which is of the form $(*)$.

Let $k \geq 0$ and $\left(i_{1}, \ldots, i_{k}\right)$ be a sequence with $1 \leq i_{1}<\cdots<i_{k} \leq N$. Then by Lemma 5.3, we have

$$
\begin{aligned}
\Delta_{\wedge}^{(1 k)}\left(u_{i_{k}} \wedge \cdots \wedge u_{i_{1}}\right) & =(-q)^{k(k-1) / 2} \Delta^{\left(1^{k}\right)}\left(u_{i_{1}} \wedge \cdots \wedge u_{i_{k}}\right) \\
& =(-q)^{k(k-1) / 2} \sum_{\sigma \in \Im_{k}}(-q)^{\ell(\sigma)} u_{i_{\sigma 1}} \otimes \cdots \otimes u_{i_{\sigma k}} \\
& =(-q)^{k(k-1) / 2} \sum_{\sigma \in \Im_{k}}(-q)^{-\ell(\sigma)} u_{i_{\sigma k}} \otimes \cdots \otimes u_{i_{\sigma 1}} .
\end{aligned}
$$

For a skew partition $\lambda / \mu$ and $S \in \operatorname{Row}_{\lambda / \mu}\left(\omega_{0}\right)$, the image of $\xi_{S}$ by the coproduct 
$\Delta: \bigwedge_{\lambda / \mu} V \rightarrow T_{\lambda / \mu} V$ is of the form $q^{*} \sum_{\sigma \in \mathfrak{S}_{\lambda-\mu}}(-q)^{-\ell(\sigma)} \zeta_{\sigma S}$, where $\omega_{0} \in \mathfrak{S}_{N}$ is the permutation of maximal length. Note that $\sigma S \geq S, \sigma S \leq S$, and $\sigma S \neq S$ for any $\sigma \in \mathfrak{S}_{\lambda-\mu} \backslash\{$ id $\}$.

Proposition 6.13. Let $\lambda / \mu$ be a skew partition. Then $L_{\lambda / \mu} Y\left(\omega_{0}\right)=\left\{d_{\lambda / \mu}(V)\left(\xi_{S}\right) \mid S \in\right.$ $\left.\mathrm{St}_{\lambda / \mu} Y\left(\omega_{0}\right)\right\}$ is linearly independent.

Proof. We denote by $\bigwedge_{\lambda / \mu}^{\prime} V\left(\right.$ resp. $\left.S_{\tilde{\lambda} / \tilde{\mu}}^{\prime} V\right)$ the submodule of $\bigwedge_{\lambda / \mu} V\left(\right.$ resp. $\left.S_{\tilde{\lambda} / \tilde{\mu}} V\right)$ generated by $\left\{\xi_{S} \mid S \in \mathrm{St}_{\lambda / \mu} Y\left(\omega_{0}\right)\right\}$ (resp. $\left\{\eta_{S} \mid S \in \mathrm{St}_{\lambda / \mu} Y\left(\omega_{0}\right)\right\}$ ). We denote the composite map

$$
\bigwedge_{\lambda / \mu}^{\prime} V \subset \bigwedge_{\lambda / \mu} V \stackrel{d_{\lambda / \mu}(V)}{\longrightarrow} S_{\tilde{\lambda} / \tilde{\mu}} V \stackrel{p}{\longrightarrow} S_{\tilde{\lambda} / \tilde{\mu}}^{\prime} V
$$

by $H$, where $p$ is the projection via the standard basis $\left\{\eta_{S} \mid S \in \operatorname{Tab}_{\lambda / \mu} Y\left(\omega_{0}\right), S\right.$ is column-standard $\}$ of $S_{\tilde{\lambda} / \tilde{\mu}} V$. Since both $\bigwedge_{\lambda / \mu}^{\prime} V$ and $S_{\tilde{\lambda} / \tilde{\mu}}^{\prime} V$ have bases indexed by the same totally order set $\left(\operatorname{St}_{\lambda / \mu} Y\left(\omega_{0}\right), \leq_{\text {lex, } \omega_{0}}\right)$, we can express $H$ by means of a square matrix with respect to these bases. It suffices to show that this matrix is upper triangular and each diagonal entry is of the form $q^{*}$. By Lemma 6.12 and the preceding observation, we have an expression

$$
d_{\lambda / \mu}(V)\left(\xi_{S}\right)=q^{*} \eta_{S}+\sum_{S^{\prime} \geq S, S^{\prime} \neq S} c_{S^{\prime}} \eta_{S^{\prime}}
$$

for $S \in \operatorname{St}_{\lambda / \mu} Y\left(\omega_{0}\right)$. For each $S^{\prime}$, we have $\eta_{S^{\prime}}=q^{*} \eta_{\left(s^{\prime}\right)}$, where $\left(S^{\prime}\right)^{\circ}$ is the columnstandardization of $S^{\prime}$ with respect to the order of $Y\left(\omega_{0}\right)$. Hence we have

$$
H\left(\xi_{S}\right)=q^{*} \eta_{S}+\sum_{S^{\prime} \geq S, S^{\prime} \neq S,\left(S^{\prime}\right)^{\circ} \in S_{\lambda} / \mu} c_{\left(\omega_{0}\right)}^{\prime} c_{\left(S^{\prime}\right)} \cdot Y_{\left(S^{\prime}\right)^{\circ}} .
$$

If $S^{\prime}$ appears in the sum and $S^{\prime} \leq S$, then $S^{\prime}$ is not row-standard. Hence $S^{\prime} \neq\left(S^{\prime}\right)^{\circ}$, since $\left(S^{\prime}\right)^{\circ}$ is row-standard. So we have $\left(S^{\prime}\right)^{\circ}<{ }_{\omega} S^{\prime} \leq{ }_{\omega} S$. If $S^{\prime}>S$, then we have $\left(S^{\prime}\right)^{\circ}<{ }_{\omega} S$, too.

For $i \geq 1$, we denote the sequence $(0, \ldots, 0, \stackrel{i}{1}, 0, \cdots)$ by $\varepsilon_{i}$, and we define $\alpha_{i}=\varepsilon_{i}-\varepsilon_{i+1}$. For a skew partition $\lambda / \mu$, we define the subset $S_{\square}(\lambda / \mu)$ of $\Omega^{+}$by:

$$
S_{\square}(\lambda / \mu)=\left\{v \in \Omega^{+} \mid{ }^{\exists} t \in N,{ }^{\exists} k \in N_{0} k<\lambda_{t+1}-\mu_{t} \text { and } v=\gamma+\left(\gamma_{t+1}-k\right) \cdot \alpha_{t}\right\},
$$

where $\quad \gamma=\lambda-\mu$.

Definition 6.14. Let $\lambda / \mu$ be a skew partition, and $\nu \in S_{\square}(\lambda / \mu)$. We have $v=\lambda-\mu+r \cdot \alpha_{t}$ for some $r, t \geq 0$. Then we define the box map $\square_{\lambda / \mu}^{v}: \bigwedge_{v} V \rightarrow \bigwedge_{\lambda / \mu} V$ to be the composite map 


$$
\begin{gathered}
\bigwedge_{v} V=\bigwedge_{\gamma_{1}} V \otimes \cdots \otimes \bigwedge_{\gamma_{t-1}} V \otimes \bigwedge_{\gamma_{t}+r} V \otimes \bigwedge_{\gamma_{t+1}-r} V \otimes \bigwedge_{\gamma_{t+2}} V \otimes \cdots \otimes \bigwedge_{\gamma_{s}} V \\
\downarrow \\
\mid 1 \otimes \cdots \otimes 1 \otimes \square \otimes 1 \otimes \cdots \otimes 1 \\
\bigwedge_{\lambda / \mu} V=\bigwedge_{\gamma_{1}} V \otimes \cdots \otimes \bigwedge_{\gamma_{t-1}} V \otimes \bigwedge_{\gamma_{t}} V \otimes \bigwedge_{\gamma_{t+1}} V \otimes \bigwedge_{\gamma_{t+2}} V \otimes \cdots \otimes \bigwedge_{\gamma_{s}} V,
\end{gathered}
$$

where $\gamma=\lambda-\mu$ and $\square$ is the composite map

$$
\bigwedge_{\gamma_{t}+r} V \otimes \bigwedge_{\gamma_{t+1}-r} V \stackrel{\Delta \otimes 1}{\longrightarrow} \bigwedge_{\gamma_{t}} V \otimes \bigwedge_{r} V \otimes \bigwedge_{\gamma_{t+1}-r} V \stackrel{1 \otimes m}{\longrightarrow} \bigwedge_{\gamma_{t}} V \otimes \bigwedge_{\gamma_{t+1}} V
$$

The sum of these box maps $\sum_{v \in S_{\square}(\lambda / \mu)} \bigwedge_{\nu} V \rightarrow \bigwedge_{\lambda / \mu} V$ is denoted by $\square_{\lambda / \mu}$.

LEMMA 6.15 (cf. [4, Lemma II.2.9]). Let $\lambda=\left(\lambda_{1}, \lambda_{2}\right)$ and $\mu=\left(\mu_{1}, \mu_{2}\right)$ be partitions with $\lambda \supset \mu$. We set $\gamma=\lambda-\mu$. Let $a$ and $b$ be non-negative integers with $a+b<\lambda_{2}-\mu_{1}$. Then the image of the composite map

$$
\begin{gathered}
\bar{\square}_{(a, b)}: \bigwedge_{a} V \otimes \bigwedge_{\gamma_{1}-a+\gamma_{2}-b} V \otimes \bigwedge_{b} V \stackrel{1 \otimes \Delta \otimes 1}{\longrightarrow} \bigwedge_{a} V \otimes \bigwedge_{\gamma_{1}-a} V \otimes \bigwedge_{\gamma_{2}-b} V \otimes \bigwedge_{b} V \\
\stackrel{m \otimes m}{\longrightarrow} \bigwedge_{\gamma_{1}} V \otimes \bigwedge_{\gamma_{2}} V=\bigwedge_{\lambda / \mu} V
\end{gathered}
$$

is contained in $\operatorname{Im} \square_{\lambda / \mu}$.

Proof. We use induction on $a$. If $a=0$, then we have $\left(\gamma_{1}+\gamma_{2}-b, b\right) \in S_{\square}(\lambda / \mu)$, and $\bar{\square}_{(a, b)}=\square_{\lambda / \mu}^{\left(\gamma_{1}+\gamma_{2}-b, b\right)}$. by assumption. So the assertion is clear. We consider the case $a>0$. For each $i \in[0, a]$, we denote by $\Delta(i)$ the composite map

$$
\begin{gathered}
\bigwedge_{a} V \otimes \bigwedge_{|\gamma|-a-b} V \stackrel{\Delta \otimes \Delta}{\longrightarrow} \bigwedge_{i} V \otimes \bigwedge_{a-i} V \otimes \bigwedge_{\gamma_{1}-i} V \otimes \bigwedge_{\gamma_{2}-a+i-b} V \\
\stackrel{1 \otimes \varphi_{\bigwedge V} \otimes 1}{\longrightarrow} \bigwedge_{i} V \otimes \bigwedge_{\gamma_{1}-i} V \otimes \bigwedge_{a-i} V \otimes \bigwedge_{\gamma_{2}-a+i-b} V .
\end{gathered}
$$

Then, we have a commutative diagram

$$
\begin{array}{cc}
\bigwedge_{a} V \otimes \bigwedge_{|\gamma|-a-b} V \longrightarrow & \bigwedge_{|\gamma|-b} V \\
\downarrow_{\wedge V \otimes \wedge V}=\sum_{i=0}^{a} \Delta(i) & \left.\right|^{\Delta} \\
\sum_{i=0}^{a} \bigwedge_{i} V \otimes \bigwedge_{\gamma_{1}-i} V \otimes \bigwedge_{a-i} V \otimes \bigwedge_{\gamma_{2}-a+i-b} V \stackrel{m \otimes m}{\longrightarrow} \bigwedge_{\gamma_{1}} V \otimes \bigwedge_{\gamma_{2}-b} V
\end{array}
$$

since $m$ is a homomorphism of coalgebras. Note that $\bar{\square}_{(a, b)}$ is nothing but the composite map 


$$
\begin{gathered}
\bigwedge_{a} V \otimes \bigwedge_{|\gamma|-a-b} V \otimes \bigwedge_{b} V \stackrel{\Delta(a) \otimes 1}{\longrightarrow} \bigwedge_{a} V \otimes \bigwedge_{\gamma_{1}-a} V \otimes \bigwedge_{\gamma_{2}-b} V \otimes \bigwedge_{b} V \\
\stackrel{m \otimes m}{\longrightarrow} \bigwedge_{\gamma_{1}} V \otimes \bigwedge_{\gamma_{2}} V=\bigwedge_{\lambda / \mu} V .
\end{gathered}
$$

On the other hand, the image of the composite map

$$
\begin{gathered}
\bigwedge_{a} V \otimes \bigwedge_{|\gamma|-a-b} V \otimes \bigwedge_{b} V \stackrel{m \otimes 1}{\longrightarrow} \bigwedge_{|\gamma|-b} V \otimes \bigwedge_{b} V \stackrel{\Delta \otimes 1}{\longrightarrow} \bigwedge_{\gamma_{1}} V \otimes \bigwedge_{\gamma_{2}-b} V \otimes \bigwedge_{b} V \\
\stackrel{1 \otimes m}{\longrightarrow} \bigwedge_{\gamma_{1}} V \otimes \bigwedge_{\gamma_{2}} V=\bigwedge_{\lambda / \mu} V
\end{gathered}
$$

is clearly contained in $\operatorname{Im} \square_{\lambda / \mu}$. Hence, it suffices to show that the image of the composite map

$$
\begin{gathered}
f_{i}: \bigwedge_{a} V \otimes \bigwedge_{|\gamma|-a-b} V \otimes \bigwedge_{b} V \stackrel{\Delta(i) \otimes 1}{\longrightarrow} \bigwedge_{i} V \otimes \bigwedge_{\gamma_{1}-i} V \otimes \bigwedge_{a-i} V \otimes \bigwedge_{\gamma_{2}-a+i-b} V \otimes \bigwedge_{b} V \\
\stackrel{m \otimes m \otimes 1}{\longrightarrow} \bigwedge_{\gamma_{1}} V \otimes \bigwedge_{\gamma_{2}-b} V \otimes \bigwedge_{b} V \stackrel{1 \otimes m}{\longrightarrow} \bigwedge_{\gamma_{1}} V \otimes \bigwedge_{\gamma_{2}} V=\bigwedge_{\lambda / \mu} V
\end{gathered}
$$

is contained in $\operatorname{Im} \square_{\lambda / \mu}$ for $i \in[0, a-1]$, because $\bar{\square}_{(a, b)}=f_{a}$ and $\operatorname{Im}\left(\sum_{i=0}^{a} f_{i}\right) \subset \operatorname{Im} \square_{\lambda / \mu}$ by the commutativity of $(*)$. Consider the diagram

$$
\begin{aligned}
& \bigwedge_{a-i} V \otimes \bigwedge_{|\gamma|-a-b} V \longrightarrow \bigwedge_{|\gamma|-a-b} V \otimes \bigwedge_{a-i} V \\
& 1 \otimes \Delta \\
& \bigwedge_{a-i} V \otimes \bigwedge_{\gamma_{1}-i} V \otimes \bigwedge_{\gamma_{2}-a+i-b} V \\
& \downarrow \varphi_{\wedge v} \otimes 1 \\
& \bigwedge_{\gamma_{1}-i} V \otimes \bigwedge_{a-i} V \otimes \bigwedge_{\gamma_{2}-a+i-b} V \quad \underset{1 \otimes \varphi_{\bigwedge^{V}}}{\longrightarrow} \bigwedge_{\gamma_{1}-i} V \otimes \bigwedge_{\gamma_{2}-a+i-b} V \otimes \bigwedge_{a-i} V \\
& m \otimes 1 \bigwedge_{\gamma_{1}-i} V \otimes \bigwedge_{\gamma_{2}-b} V .
\end{aligned}
$$

The upper square commutes, since $\left(\bigwedge V, \varphi_{\bigwedge}\right)$ is a YB coalgebra. Since $\varphi_{\bigwedge}$ equals $\left(-q^{2}\right)^{*} \psi_{\wedge_{V}}$ on $\bigwedge_{a-i} V \otimes \bigwedge_{\gamma_{2}-a+i-b} V$, the lower triangle commutes up to unit thanks to Proposition 4.11 (1). Together with the associativity of the algebra $\wedge V$, these commutativity of the diagram above yields that $f_{i}$ is a unit multiple of the composite map

$$
\begin{aligned}
& \bigwedge_{a} V \otimes \bigwedge_{|\gamma|-a-b} V \otimes \bigwedge_{b} V \stackrel{\Delta \otimes 1}{\longrightarrow} \bigwedge_{i} V \otimes \bigwedge_{a-i} V \otimes \bigwedge_{|\gamma|-a-b} V \otimes \bigwedge_{b} V \\
& \stackrel{1 \otimes \varphi_{\bigwedge^{V}} \otimes 1}{\longrightarrow} \bigwedge_{i} V \otimes \bigwedge_{|\gamma|-a-b} V \otimes \bigwedge_{a-i} V \otimes \bigwedge_{b} V
\end{aligned}
$$


$\stackrel{1 \otimes m}{\longrightarrow} \bigwedge_{i} V \otimes \bigwedge_{|\gamma|-i-(a+b-i)} V \otimes \bigwedge_{a+b-i} V \stackrel{\bar{\square}_{(i, a+b-i)}}{\longrightarrow} \bigwedge_{\gamma_{1}} V \otimes \bigwedge_{\gamma_{2}} V=\bigwedge_{\lambda / \mu} V$, whose image is contained in $\operatorname{Im} \square_{\lambda / \mu}$ by the induction hypothesis.

We emphasize that the proof above is a slight modification of the proof of $[4$, Lemma II.2.9]. Similarly, we can prove the following lemma using the idea of the proof of $[4$, Proposition II.2.8] and the properties of YB bialgebras $\bigwedge V$ and $S V$ from $\S 4$.

LEMMA 6.16. Let $\lambda / \mu=\left(\lambda_{1}, \lambda_{2}\right) /\left(\mu_{1}, \mu_{2}\right)$ be a skew shape consisting of two rows, and $v \in S_{\square}(\lambda / \mu)$. Then, the composite map

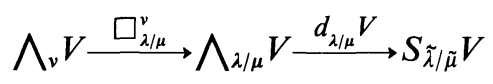

is zero.

The proof is left to the reader.

Lemma 6.17. Let $\lambda / \mu$ be a skew partition, and $v \in S_{\square}(\lambda / \mu)$. Then the composite map

$$
\bigwedge_{\nu} V \stackrel{\square_{\lambda / \mu}^{v}}{\longrightarrow} \bigwedge_{\lambda / \mu} V \stackrel{d_{\lambda / \mu}(V)}{\longrightarrow} S_{\tilde{\lambda} / \tilde{\mu}} V
$$

is zero.

Proof. We set $v=\lambda-\mu+t \cdot \alpha_{k}, \lambda^{+}=\left(\lambda_{1}, \ldots, \lambda_{t-1}\right), \mu^{+}=\left(\mu_{1}, \ldots, \mu_{t-1}\right), \quad \lambda^{0}=$ $\left(\lambda_{t}, \lambda_{t+1}\right), \quad \mu^{0}=\left(\mu_{t}, \mu_{t+1}\right), \quad \lambda^{-}=\left(\lambda_{t+2}, \ldots, \lambda_{s}\right), \quad$ and $\mu^{-}=\left(\mu_{t+2}, \ldots, \mu_{s}\right)$, where $s=$ $\ell(\lambda / \mu)$. We have a reduced expression $\chi_{\lambda / \mu}=\rho \cdot \chi_{\lambda^{+} / \mu^{+}} \cdot \chi_{\lambda^{0} / \mu^{0}} \cdot \chi_{\lambda^{-} / \mu^{-}}$with unique $\rho \in$

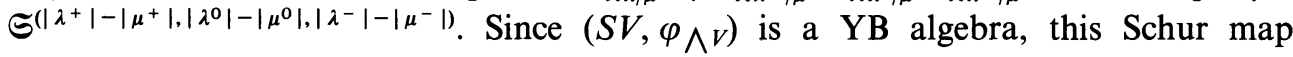
$d_{\lambda / \mu}(V)$ coincides with the composite map

$$
\begin{aligned}
& \bigwedge_{\lambda / \mu} V=\bigwedge_{\lambda^{+} / \mu^{+}} V \otimes \bigwedge_{\lambda^{0} / \mu^{0}} V \otimes \bigwedge_{\lambda^{-} / \mu^{-}} V \\
& \stackrel{d_{\lambda^{+} / \mu^{+}} \otimes d_{\lambda^{0} / \mu^{0}} \otimes d_{\lambda^{-} / \mu^{-}}^{\longrightarrow}}{\longrightarrow} S_{\tilde{\lambda}+/ \tilde{\mu}+} V \otimes S_{\tilde{\lambda} 0 / \tilde{\mu}^{0}} V \otimes S_{\tilde{\lambda}-/ \tilde{\mu}-} V \stackrel{m}{\longrightarrow} S_{\tilde{\lambda} / \tilde{\mu}} V,
\end{aligned}
$$

where $m$ is the restriction of the multiplication $T_{\lambda_{1}} S V \otimes T_{\lambda_{1}} S V \otimes T_{\lambda_{1}} S V \rightarrow T_{\lambda_{1}} S V$ defined in Proposition 4.2 (which agrees with $m_{S V}^{\otimes \lambda_{1}} \circ \varphi_{S V}(w)$, where $m_{S V}$ is the multiplication $S V \otimes S V \otimes S V \rightarrow S V$ and $w \in \Im_{3 \lambda_{1}}$ is given by $w\left(i \lambda_{1}+j\right)=3 j+i-2$ for $i=0,1,2$ and $j=1, \ldots, \lambda_{1}$. Observe that the restriction of $\varphi_{S V}(w)$ to $S_{\tilde{\lambda}^{+} / \tilde{\mu}^{+}} V \otimes S_{\tilde{\lambda}^{0} / \tilde{\mu}^{0}} V \otimes S_{\tilde{\lambda}^{-} / \tilde{\mu}^{-}} V$ is induced by $\left.\left(q^{-2} \beta\right)(w): T_{\tilde{\lambda}^{+} / \tilde{\mu}^{+}} V \otimes T_{\tilde{\lambda}^{0} / \tilde{\mu}^{0}} V \otimes T_{\tilde{\lambda}_{-} / \tilde{\mu}^{-}} V \rightarrow T_{\tilde{\lambda} / \tilde{\mu}} V\right)$. On the other hand, the box map $\square_{\lambda / \mu}^{v}$ is nothing but $1 \otimes \square_{\lambda 0 / \mu^{0}}^{v^{0}} \otimes 1$, where $v^{0}=\left(v_{t}, v_{t+1}\right)$. Therefore, the lemma is clear by Lemma 6.16 .

LEMMA 6.18. Let $\lambda / \mu$ be a skew partition, $\sigma \in \mathfrak{S}_{N}$, and $S \in \operatorname{Row}_{\lambda / \mu} Y(\sigma) \backslash \operatorname{St}_{\lambda / \mu} Y(\sigma)$. Then, there exist $S_{1}^{\prime}, \ldots, S_{r}^{\prime} \in \operatorname{Row}_{\lambda / \mu} Y(\sigma)(r \in N)$ with $S_{i}^{\prime}<_{\sigma} S$ for each $i \in[1, r]$ such that 
$\xi_{S}-\sum_{i} c_{i} \xi_{S_{i}^{\prime}} \in \operatorname{Im} \square_{\lambda / \mu}$ for some $c_{i} \in Z\left[q, q^{-1}\right]$.

Proof. The proof is quite similar to that of [4, II. Lemma 2.15] (the case $q=1$ ), so we only point out some non-trivial differences. The proof in [4] depends on the fact corresponding to (2.3) and (2.4) for the case $q=1$, while we can use these without much problem, since $\pm q^{*}$ is a unit by our assumption. It also depends on the following fact: (*) Let $s, t \in N$ and $i_{1}, \ldots, i_{s+t}$ be distinct integers in $[1, N]$. Then, the image of $u_{i_{1}} \wedge \cdots \wedge u_{i_{s+t}}$ by the map $\Delta_{\bigwedge V}^{(s, t)}: \bigwedge_{s+t} V \rightarrow \bigwedge_{s} V \otimes \bigwedge_{t} V$ equals

$$
\sum_{\pi \in \mathbb{S}^{(s, t)}} \pm q^{*} u_{i_{\pi 1}} \wedge \cdots \wedge u_{i_{\pi s}} \otimes u_{i_{\pi(s+1)}} \wedge \cdots \wedge u_{i_{\pi t}}
$$

(for the case $q=1$ ).

Let us prove $(*)$. If $i_{1}<\cdots<i_{s+t}$ (this case corresponds to the case $\sigma=\mathrm{id}$ ), then

$$
\Delta_{\wedge}^{(s, t)}\left(u_{i_{1}} \wedge \cdots \wedge u_{i_{s+t}}\right)=\sum_{\pi \in \mathbb{S}^{(s, t)}}(-q)^{\ell(\pi)} u_{i_{\pi 1}} \wedge \cdots \wedge u_{i_{\pi s}} \otimes u_{i_{\pi(s+1)}} \wedge \cdots \wedge u_{i_{\pi t}}
$$

by Proposition 4.8 and (5.4). The general case follows from (2.4). Since the coefficients in the sum are units, we can use $(*)$. We have generalized [4, Lemma II.2.9], which is used in the proof, to Lemma 6.15, and we can use this generalized version. We leave the complete modifications to the interested reader.

THEOREM 6.19. Let $\lambda / \mu$ be a skew partition. Then the Schur module $L_{\lambda / \mu} V$ is an $R$-free module with $L_{\lambda / \mu} Y(\sigma)=\left\{d_{\lambda / \mu}(V)\left(\xi_{S}\right) \mid S \in \mathrm{St}_{\lambda / \mu} Y(\sigma)\right\}$ its free basis for any $\sigma \in \mathfrak{\Xi}_{N}$. The sequence of $S E^{\vee}$-comodules

$$
\sum_{v \in S_{\square}(\lambda / \mu)} \bigwedge_{v} V \stackrel{\square_{\lambda / \mu}}{\longrightarrow} \bigwedge_{\lambda / \mu} V \stackrel{d_{\lambda / \mu}(V)}{\longrightarrow} L_{\lambda / \mu} V \longrightarrow 0
$$

is exact. The Schur module $L_{\lambda / \mu} V$ is a direct summand of $S_{\lambda / \tilde{\mu}} V$ via the natural inclusion, as an R-module.

ProOF. Clearly, $d_{\lambda / \mu}(V): \bigwedge_{\lambda / \mu} V \rightarrow L_{\lambda / \mu} V$ is surjective. With any $a=\sum_{S \in \operatorname{Row}_{\lambda / \mu} Y(\sigma)} c_{S} \xi_{S} \in$ $\bigwedge_{\lambda / \mu} V$, we associate a non-negative integer $N(a)=\sum_{c_{S} \neq 0} 2^{\text {ht(S) }}$, where $\mathrm{ht}(S)$ is the height of $S$ with respect to the order $\leq_{\text {lex, } \sigma}$ of $\operatorname{Row}_{\lambda / \mu} Y(\sigma)$. We claim that $a$ can be expressed in the form $a=a_{0}+\sum_{S \in \mathrm{St}_{\lambda / \mu} \mathrm{Y}(\sigma)} c_{S}^{\prime} \xi_{S}$ with $a_{0} \in \operatorname{Im} \square_{\lambda / \mu}$. We prove this by induction on $N(a)$. If each $S$ appearing in the sum with $c_{S} \neq 0$ is standard, then there is nothing to prove. So we may assume that some $S$ is not standard. For such an $S$, we have an expression $\xi_{S}=\sum_{i} c_{S_{i}^{\prime}}^{\prime \prime} \cdot S_{i}^{\prime}+a_{0}^{\prime \prime}$ for some $a_{0}^{\prime \prime} \in \operatorname{Im} \square_{\lambda / \mu}$ and $S_{i}^{\prime}<_{\sigma} S$ by Lemma 6.18. Then we have $a=c_{S} a_{0}^{\prime \prime}+\left(a-c_{S} a_{0}^{\prime \prime}\right)$, and $N\left(a-c_{S} a_{0}^{\prime \prime}\right)<N(a)$. So the claim is now clear by the induction hypothesis. Since we have $d_{\lambda / \mu}(a)=\sum_{S} c_{S}^{\prime} d_{\lambda / \mu}\left(\xi_{S}\right)$ by Lemma 6.17, $L_{\lambda / \mu} Y(\sigma)$ generates $L_{\lambda / \mu} V$ for any $\sigma$. By Proposition 6.13, $L_{\lambda / \mu} Y\left(\omega_{0}\right)$ is a free basis of $L_{\lambda / \mu} V$. Since $\# L_{\lambda / \mu} Y(\sigma)=\# L_{\lambda / \mu} Y\left(\omega_{0}\right)$, the first assertion is now clear. If $a \in \operatorname{Ker} d_{\lambda / \mu}$, then we have $0=d_{\lambda / \mu}(a)=\sum_{S} c_{S}^{\prime} \cdot d_{\lambda / \mu}\left(\xi_{S}\right)$. Since $d_{\lambda / \mu}\left(\xi_{S}\right)$ are linearly independent, we have $c_{S}^{\prime}=0$ for all $S$. Hence, $a=a_{0} \in \operatorname{Im} \square_{\lambda / \mu}$, and the second assertion is proved. As we have seen in the 
proof of Proposition 6.13, $L_{\lambda / \mu} V+S_{\tilde{\lambda} \mid \tilde{\mu}}^{\prime \prime} V=S_{\tilde{\lambda} / \tilde{\mu}}$, where $S_{\tilde{\lambda} / \tilde{\mu}}^{\prime \prime} V$ is the $R$-span of the set $\left\{\eta_{S} \mid S \in \operatorname{Tab}_{\lambda / \mu} Y(\mathrm{id}), S\right.$ is column-standard but is not row standard $\}$. This sum must be a direct sum by the first assertion, so the last assertion is now clear.

COROLlaRY 6.20. Let $f: R \rightarrow R^{\prime}$ be a homomorphism of commutative rings. Then we have an isomorphism of $S\left(E^{\prime}\right)^{\vee}$-comodules $L_{\lambda}\left(R^{\prime} \otimes_{R} V\right) \simeq R^{\prime} \otimes_{R} L_{\lambda} V$, where $E^{\prime}=R^{\prime} \otimes_{R} E$, and $R^{\prime} \otimes_{R} V$ is Jimbo's YB-pair obtained by base change.

Proof. Note that $\bigwedge_{\lambda / \mu}$ and $\sum_{\nu} \bigwedge_{\nu}$ are compatible with base changes, and that $\square_{\lambda / \mu}$ is defined over $Z\left[Q, Q^{-1}\right]$, where $Q$ is an indeterminate. By Theorem 6.19 , we have an isomorphism Coker $\square_{\lambda / \mu} \simeq L_{\lambda / \mu} V$. Since cokernels are compatible with base change, the assertion is clear.

We shall now define the Weyl modules of $V$.

Definition 6.21. Let $\lambda / \mu$ be a skew partition. Let $d_{\lambda / \mu}^{\prime}(V)$ be the composite map

$$
\begin{aligned}
& D_{\lambda / \mu} V \stackrel{\Delta \otimes \cdots \otimes \Delta}{\longrightarrow} T_{\lambda / \mu} V=T_{|\lambda|-|\mu|} V \stackrel{\left(q^{-2} \beta\right)\left(\chi_{\lambda / \mu}\right)}{\longrightarrow} T_{|\lambda|-|\mu|} V \\
& =T_{\tilde{\lambda} / \tilde{\mu}} V \stackrel{p \otimes \cdots \otimes p}{\longrightarrow} \bigwedge \tilde{\lambda} / \tilde{\mu} V .
\end{aligned}
$$

It is called the Weyl map. We denote the image $\operatorname{Im} d_{\lambda / \mu}^{\prime}(V)$ of $d_{\lambda / \mu}^{\prime}(V)$ by $K_{\lambda / \mu} V$ and called it the Weyl module of $V$ with respect to the skew partition $\lambda / \mu$.

With the natural identifications $\bigwedge \tilde{\lambda} / \tilde{\mu} V=\left(\bigwedge_{\tilde{\lambda} / \tilde{\mu}}{ }^{t} V\right)^{*}$ and $D_{\lambda / \mu} V=\left(S_{\lambda / \mu}{ }^{t} V\right)^{*}$, we can identify $d_{\lambda / \mu}^{\prime}(V)$ with $\left.\left(d_{\tilde{\lambda} / \tilde{\mu}}{ }^{t} V\right)\right)^{*}$, since $\chi_{\bar{\lambda} / \tilde{\mu}}^{\tilde{\mu}}=\chi_{\lambda / \mu}$ so that $\left(\left(q^{-2 t} \beta\right)\left(\chi_{\tilde{\lambda} / \tilde{\mu}}\right)\right)^{*}=\left(q^{-2} \beta\right)\left(\chi_{\lambda / \mu}\right)$. Since $L_{\tilde{\lambda} / \tilde{\mu}}{ }^{t} V$ is a direct summand of $S_{\lambda / \mu}{ }^{t} V$, we have a standard isomorphism $K_{\lambda / \mu} V \simeq\left(L_{\tilde{\lambda} / \tilde{\mu}}^{t} V\right)^{*}$. In particular, $K_{\lambda / \mu} V$ is a free $R$-module with the same rank as $L_{\tilde{\lambda} / \tilde{\mu}} V$.

To describe a presentation and the standard basis of $K_{\lambda / \mu} V$, we need additional notation. We define $\Omega_{k}^{+}=\left\{\alpha \in \Omega^{+}|| \alpha \mid=k\right\}$ for $k \geq 0$. We denote the dual basis of $X$ by ${ }^{t} X=\left\{v_{1}, \ldots, v_{N}\right\}$. So we have a basis $\left\{v^{\alpha} \mid \alpha \in \Omega_{k}^{+}, \ell(\alpha) \leq N\right\}$ of $S_{k}{ }^{t} V$ for $k \geq 0$, where $v^{\alpha}=v_{1}^{\alpha_{1}} \cdots v_{N}^{\alpha_{N}}$. The dual basis $\left\{u^{(\alpha)} \mid \alpha \in \Omega_{k}^{+}, \ell(\alpha) \leq N\right\}$ is a basis of $D_{k} V$, where $u^{(\alpha)}$ is the basis corresponding to $v^{\alpha}$. For $i \in[1, N]$ and $k \geq 0$, we denote $u^{k \cdot \varepsilon_{i}}$ by $u_{i}^{(k)}$. Since the product of $D V$ is the dual of the coproduct of $S^{t} V$, we have $u_{i}^{k}=[k]_{q-2}^{!} u_{i}^{(k)}$ in $D_{k} V$ by Lemma 5.4 applied to $S^{t} V$, where $u_{i}$ on the left hand side is $u_{i}^{(1)} \in D_{1} V=V$. We call $u_{i}^{(k)}$ the $k$-th divided power of $u_{i}$.

LEMMA 6.22. Let $k, \alpha$ and $v$ be as in Lemma 5.4. Then we have $u^{(\alpha)}=u_{1}^{\left(\alpha_{1}\right)} \cdots u_{N}^{\left(\alpha_{N}\right)}$. If $\sigma \in \mathfrak{S}^{\alpha}$, then we have

$$
u_{v \sigma^{-1} 1} \cdots u_{v \sigma^{-1} k}=q^{-\ell(\sigma)} u^{\alpha}=\left(\prod_{i=1}^{N}\left[\alpha_{i}\right]_{q^{-2}}^{!}\right) q^{-\ell(\sigma)} u^{(\alpha)}
$$

in $D_{k} V$. 
Proof. The second assertion follows immediately by Lemma 5.4, since the product of $D V$ is the dual of the coproduct of $S^{t} V$. To prove the first assertion, we may assume that the base ring $R$ is $Z\left[q, q^{-1}\right]$ with $q$ transcendental over $Z$. By the observation above, we have

$$
u^{\alpha}=u_{1}^{\alpha_{1}} \cdots u_{N}^{\alpha_{N}}=\left(\prod_{i=1}^{N}\left[\alpha_{i}\right]_{q^{-2}}^{!}\right) u_{1}^{\left(\alpha_{1}\right)} \cdots u_{N}^{\left(\alpha_{N}\right)} .
$$

On the other hand, we have $u^{\alpha}=\left(\prod_{i}\left[\alpha_{i}\right]_{q^{-2}}^{!}\right) u^{(\alpha)}$ by the first assertion. Since $\prod_{i}\left[\alpha_{i}\right]_{q^{-2}}^{!}$

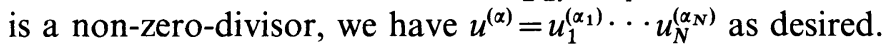

For a skew partition $\lambda / \mu$ and $S \in \operatorname{Tab}_{\lambda / \mu} X$, we set

$$
\xi^{\prime}(S)=q^{-\left(\ell\left(\sigma^{(1)}\right)+\cdots+\ell\left(\sigma^{(s)}\right)\right)} u^{\left(\alpha^{(1)}\right)} \otimes \cdots u^{\left(\alpha^{(s)}\right)} \in D_{\lambda / \mu} V,
$$

where $s=\ell(\lambda-\mu), \alpha^{(i)}=\left(\alpha_{1}^{(i)}, \ldots, \alpha_{N}^{(i)}\right)$ is given by $\alpha_{t}^{(i)}=\#\left\{j \mid S(i, j)=u_{t}\right\}$ for $t \in[1, N]$, and $\sigma^{(i)}$ is the unique element in $\mathfrak{S}^{\alpha^{(i)}}$ such that $S\left(i, \mu_{i}+\sigma^{(i)} j\right)$ is the $j$-th element in the sequence $\left(1^{\alpha_{1}^{(i)}}, \ldots, N^{\alpha_{N}^{(i)}}\right)$. It is clear that $\left\{\xi^{\prime}(S) \mid S \in \operatorname{Corow}_{\lambda / \mu} Y(\rho)\right\}$ is a free basis of $D_{\lambda / \mu} V$ for any $\rho \in \mathfrak{S}_{N}$.

THEOREM 6.23. Let $\lambda / \mu$ be a skew partition. Then the Weyl module $K_{\lambda / \mu} V$ is an $R$-free module with $\left\{d_{\lambda / \mu}^{\prime}(V)\left(\xi_{S}^{\prime}\right) \mid S \in \operatorname{Cost}_{\lambda / \mu} Y(\rho)\right\}$ as its free basis for any $\rho \in \Im_{N}$. The sequence of $S E^{\vee}$-comodules

$$
\cdot \sum_{v \in S_{\square}(\lambda / \mu)} D_{v} V \stackrel{\square_{\lambda / \mu}^{\prime}}{\longrightarrow} D_{\lambda / \mu} V \stackrel{d_{\lambda / \mu}^{\prime}(V)}{\longrightarrow} K_{\lambda / \mu} V \longrightarrow 0
$$

is exact, where $\square_{\lambda / \mu}^{\prime}$ is defined similarly to $\square_{\lambda / \mu}$. The Weyl module $K_{\lambda / \mu}$ is a direct summand of $\bigwedge \tilde{\lambda} / \tilde{\mu} V$ as an $R$-module via the natural inclusion.

Proof. Since the inclusion $K_{\lambda / \mu} V \varsigma \bigwedge_{\tilde{\lambda} / \tilde{\mu}} V$ can be identified with the dual of the Schur map $\left.d_{\tilde{\lambda} / \tilde{\mu}}{ }^{t} V\right): \bigwedge \tilde{\lambda} /{ }^{t} V \rightarrow L_{\tilde{\lambda} / \tilde{\mu}}{ }^{t} V$, the last assertion is trivial. The rest of the theorem is proved similarly to Theorem 6.19 (see also the proof of [4; Theorem II.3.16]. To translate this proof into our context, we need Lemma 6.22). We only remark that there is no need to prove the fact corresponding to Proposition 6.13, since we already know that $K_{\lambda / \mu} V$ is free of rank $\# \operatorname{Cost}_{\lambda / \mu} Y(\rho)=\# \operatorname{St}_{\tilde{\lambda} / \tilde{\mu}} Y(\rho)$.

COROLlaRY 6.24. Let $\lambda / \mu$ be a skew partition, and $R \rightarrow R^{\prime}$ a homomorphism of commutative rings. Then we have an $S\left(E^{\prime}\right)^{\vee}$-comodule isomorphism $K_{\lambda}\left(R^{\prime} \otimes V\right) \simeq$ $R^{\prime} \otimes K_{\lambda} V$, where $E^{\prime}=R^{\prime} \otimes E$.

7. Universality of Weyl modules. Throughout this section, $V=\left(V, \beta_{V}, \gamma_{V}\right)$ denotes Jimbo's YB triple determined by a basis $\left\{u_{1}, \ldots, u_{N}\right\}$ and $q \in R^{\times}$, and $E=(E, \beta, \gamma)$ is as in Example (2) in $\S 4$.

Let $A$ be an $R$-algebra (resp. $R$-coalgebra). We denote by $A$-mff (resp. mff- $A$, resp. $A$-cff, resp. cff- $A$ ) the category of left $A$-modules (resp. right $A$-modules, resp. left 
$A$-comodules, resp. right $A$-comodules) which are free of finite rank as $R$-modules. Let $C$ be an $R$-coalgebra which is free of finite rank as an $R$-module, and $M$ a $C$-comodule. The map $C^{*} \otimes M \rightarrow M$ given by $a \otimes u \mapsto \sum_{i} \operatorname{ev}\left(a \otimes u_{i}^{(2)}\right) u_{i}^{(1)}$ is a left action of the dual algebra $C^{*}$ of $C$ on $M$, where $\omega(u)=\sum_{i} u_{i}^{(1)} \otimes u_{i}^{(2)}$. This correspondence is an equivalence between the category of right $C$-comodules and the category of left $C^{*}$-modules, and we have an equivalence cff- $C \neg C^{*}$-mff. We will sometimes identify cff- $C$ with $C^{*}$-mff by this correspondence. The corresponding notion of the $R$-dual ()$^{*}=\operatorname{Hom}_{R}(-, R)$ : $C^{*}$-mff $\rightarrow \mathrm{mff}-C^{*}$ is the $R$-dual cff- $C \rightarrow C$-cff (see $\S 5$ ).

Since the coalgebra $S E^{\vee}$ is decomposed into the direct sum $S E^{\vee}=\bigoplus_{k \geq 0} S_{k} E^{\vee}$, we have a decomposition $M=\bigoplus_{k \geq 0} M_{k}$ for any $S E^{\vee}$-comodule $M$, where $M_{k}$ is the image of the map

$$
M \stackrel{\omega}{\longrightarrow} M \otimes S E^{\vee} \stackrel{\text { proj }}{\longrightarrow} M \otimes S_{k} E^{\vee} \stackrel{1 \otimes e}{\longrightarrow} M .
$$

Clearly, $M_{k}$ is an $S_{k} E^{\vee}$-comodule. We call $M_{k}$ the degree $k$ component of $M$. The comodule $M$ is said to be locally free of finite rank when $M_{k}$ is free of finite rank as an $R$ module for each $k \geq 0$. We denote the category of locally finite free right (resp. left) $S E^{\vee}$-comodules by $\mathscr{P}$ (resp. $\mathscr{Q}$ ). It is clear that $\mathscr{P} \simeq \prod_{k \geq 0}$ cff- $S_{k} E^{\vee}$ and $\mathscr{Q} \simeq \prod_{k \geq 0} S_{k} E^{\vee}$-cff. The categories $\mathscr{P}$ and $\mathscr{Q}$ are additive, and are closed under extensions and tensor products. Taking the product of the dual ( )* : cff- $S_{k} E^{\vee} \rightarrow S_{k} E^{\vee}$-cff, we obtain the graded dual ()$^{\dagger}:=\prod_{k \geq 0}()^{*}: \mathscr{P} \rightarrow \mathscr{Q}$. The graded dual is a contravariant equivalence of categories, and preserves tensor products. Similarly, the graded dual $\mathscr{Q} \rightarrow \mathscr{P}$ is also defined, and is also denoted by ()$^{\dagger}$. It holds that ()$^{\dagger} \circ()^{\dagger}=$ Id.

The dual algebra of $S_{k} E^{\vee}$ is $\left(S_{k} E^{\vee}\right)^{*}=D_{k} E$. Since the projection $\left(E^{\vee}\right)^{\otimes k} \rightarrow S_{k} E^{\vee}$ is nothing but the product, $D_{k} E$ is a subalgebra of $E^{\otimes k} \simeq \operatorname{End}_{R} V^{\otimes k}$ via the coproduct $\Delta_{D^{t} E}^{\left(1^{k}\right)}$. By Proposition 5.6, we have $D_{k} E \simeq\left(E^{\otimes k}\right)^{\mathfrak{B}_{k}} \simeq \operatorname{End}_{\mathfrak{B}_{k}}\left(V^{\otimes k}\right)$.

Definition 7.1. The dual algebra $D_{k} E=\operatorname{End}_{\mathfrak{B}_{\mathbf{k}}} V^{\otimes k}$ of $S_{k} E^{\vee}$ is called the Schur algebra of degree $k$.

We denote the dual basis of $\left\{u_{1}, \ldots, u_{N}\right\}$ by $\left\{v_{1}, \ldots, v_{N}\right\}$. The $R$-linear map $\rho: V \rightarrow V^{*}$ given by $\rho\left(u_{i}\right)=v_{i}$ is an element of $\mathscr{Y} \mathscr{B}_{R}\left(V,{ }^{t} V\right) \simeq \mathscr{Y} \mathscr{B}_{R}\left(V^{-1}, V^{\vee}\right)$. Clearly, we have $\rho^{*}=\rho^{-1}$. Since $S E^{\vee}$ and $S^{t} E$ are the quotients of the same bialgebra $T E^{\vee}=T^{t} E$ defined by the same biideal $\left(\operatorname{Im}\left(1-\beta_{E}^{\vee}\right)\right)=\left(\operatorname{Im}\left(1-{ }^{t} \beta_{E}\right)\right)$, they are isomorphic as bialgebras. The composite map

$$
S E^{\vee} \stackrel{\sim}{\longrightarrow} S^{t} E \stackrel{S \tau_{v^{*}, V}}{\longrightarrow} S E^{-1} \stackrel{S\left(\rho \otimes \rho^{-1}\right)}{\longrightarrow} S E^{\vee}
$$

is an anticoalgebra algebra isomorphism, and is denoted by $\operatorname{tr}$. Since $\left(\left(\rho \otimes \rho^{-1}\right)\right.$ 。 $\left.\tau_{V^{*}, V}\right)^{2}=\mathrm{id}$, we have $\operatorname{tr}^{2}=\mathrm{id}$.

Definition 7.2. The composite functor $\operatorname{tr}_{\sharp} \circ()^{\dagger}: \mathscr{P} \rightarrow \mathscr{P}$ is denoted by ()$^{\circ}$, and 
is called the contravariant dual, where $\operatorname{tr}_{\sharp}: \mathscr{Q} \rightarrow \mathscr{P}$ is the push-forward with respect to tr.

LEMMA 7.3. (1) The contravariant dual ( $)^{\circ}: \mathscr{P} \rightarrow \mathscr{P}$ is a contravariant additive functor, and preserves tensor products.

(2) $\left(()^{\circ}\right)^{2}=\mathrm{id}_{\mathscr{P}}$.

(3) $\rho: V \rightarrow V^{\circ}$ is an isomorphism of $S E^{\vee}$-comodules. $\beta_{V}^{\circ}$ is the composite map

$$
V^{\circ} \otimes V^{\circ} \stackrel{\rho^{-1} \otimes \rho^{-1}}{\longrightarrow} V \otimes V \stackrel{\beta_{V}}{\longrightarrow} V \otimes V \stackrel{\rho \otimes \rho}{\longrightarrow} V^{\circ} \otimes V^{\circ}
$$

Proof. Part (1) is obvious. Part (2) follows easily from the fact $\operatorname{tr}^{2}=1$. (3) The action of $D_{1} E=$ End $V$ on $V^{*}=V^{\circ}$ is given by $\left(u_{i} \otimes v_{j}\right) \cdot v_{k}=v_{k}\left(\operatorname{tr}^{*}\left(u_{i} \otimes v_{j}\right)\right)=v_{k} \cdot\left(u_{j} \otimes\right.$ $\left.v_{i}\right)=\delta_{j k} v_{i}$. Hence $\rho$ is an isomorphism. The map $\beta_{V}^{\circ}$ is nothing but ${ }^{t} \beta_{V}$ by definition. Since $\rho \in \mathscr{Y} \mathscr{B}_{R}\left(V,{ }^{t} V\right)$, we have completed the proof.

Proposition 7.4. The maps $T \rho: T V \rightarrow \operatorname{tr}_{\sharp} T^{t} V \simeq(T V)^{\circ}, \quad D \rho: D V \rightarrow \operatorname{tr}_{\sharp} D^{t} V \simeq$ $(S V)^{\circ}, S \rho: S V \rightarrow \operatorname{tr}_{\sharp} S^{t} V \simeq(D V)^{\circ}, \bigwedge \rho: \bigwedge V \rightarrow \operatorname{tr}_{\sharp} \bigwedge^{t} V \simeq(\bigwedge V)^{\circ}$ are isomorphism of $S E^{\vee}$-equivariant graded $\mathrm{YB}$ bialgebras, where $\simeq$ 's are the usual identifications (see $\S 5$ ).

Proof. The identifications $\simeq$ 's are isomorphisms of $S E^{\vee}$-equivariant graded YB bialgebras, thanks to the observation after Lemma 5.8. Since $\rho: V \rightarrow{ }^{t} V$ is an isomorphism of YB pairs, it suffices to show that $T \rho, D \rho, S \rho$ and $\bigwedge \rho$ are $S E^{\vee}$-homomorphisms. This is clear for $T \rho$ by Lemma 7.3. So the induced maps $S \rho$ and $\Lambda \rho$ are $S E^{\vee}$-homomorphisms. It is easy to see that $D \rho=(S \rho)^{\circ}$, so it is also an $S E^{\vee}$-homomorphism.

Corollary 7.5. For a partition $\lambda, D_{\lambda} \rho: D_{\lambda} V \rightarrow \operatorname{tr}_{\sharp} D_{\lambda}{ }^{t} V \simeq\left(S_{\lambda} V\right)^{\circ}, \bigwedge_{\lambda} \rho: \bigwedge_{\lambda} V \rightarrow$ $\operatorname{tr}_{\sharp} \bigwedge_{\lambda}{ }^{t} V \simeq\left(\bigwedge_{\lambda} V\right)^{\circ}, L_{\lambda} \rho: L_{\lambda}^{\prime} V \rightarrow \operatorname{tr}_{\sharp} L_{\lambda}{ }^{t} V \simeq\left(K_{\tilde{\lambda}} V\right)^{\circ}$ are isomorphisms of $S E^{\vee}$-comodules. We have $\left(K_{\tilde{\lambda}} \rho\right)^{\circ}=L_{\tilde{\lambda}} \rho$ and $D_{\lambda} \rho=\left(S_{\lambda} \rho\right)^{\circ}$.

REMARK 7.6. It is easy to see that $\beta_{E^{\vee}}^{ \pm}, \gamma_{E^{\vee}}^{ \pm} \in \mathscr{P}\left(\left(E^{\vee}\right)^{\otimes 2},\left(E^{\vee}\right)^{\otimes 2}\right)$, where $\left(E^{\vee}\right)^{\otimes 2}$ is the subcomodule of the right regular representation $S E_{S E^{\vee}}^{\vee}$. It follows that $S E^{\vee}$ and $S^{t} E$ are $S E^{\vee}$-equivariant YB-bialgebras. The direct sum of Schur algebras $D E=\bigoplus_{k \geq 0} D_{k} E$ is an $R$-algebra without unit. The graded duals of the left and the right regular representations $\left({ }_{D E} D E\right)^{\dagger}$ and $\left(D E_{D E}\right)^{\dagger}$ are identified with the regular representations $S E^{\vee} S E^{\vee}$ and $S E_{S E^{\vee}}^{\vee}$ respectively. Since

$$
\operatorname{tr}: S E_{S E^{\vee}}^{\vee} \longrightarrow \operatorname{tr}_{\sharp}\left(S E^{\vee} S E^{\vee}\right)
$$

is an isomorphism in $\mathscr{P}$, we have $\left.{ }_{D E} D E\right)^{\circ} \simeq S E^{\vee} S E^{\vee}$. It follows that ${ }_{D E} D E$, viewed as $S E^{\vee}$-comodule, is also an $S E^{\vee}$-equivariant YB bialgebra. The left $D E$-module $D E^{-1}$ is identified with $\left(S^{t} E_{S E^{\vee}}\right)^{\circ}$, and is also an $S E^{\vee}$-equivariant YB bialgebra.

Let $I$ be the ideal of $S E^{\vee}$ generated by $\left\{x_{i j} \mid i \neq j\right\}$. It is easy to see that $I$ is a homogeneous bi-ideal of $S E^{\vee}$. By (3.6), we have $S E^{\vee} / I=S\left(\oplus_{i=1}^{N} R t_{i}, \tau\right)$, where $t_{i}$ is the image of $x_{i i}$ in $S E^{\vee} / I$, and $\tau$ is the trivial twisting. Let us denote by $T$ the YB pair 
$\left(\oplus_{i=1}^{N} R t_{i}, \tau\right)$. The structure of $S T$ is independent of $q$, and is commutative and cocommutative. In fact, $S T=R\left[t_{1}, \ldots, t_{N}\right]$ is a polynomial ring, and each monomial $t^{\lambda}=t^{\lambda_{1}} \cdots t_{N}^{\lambda_{N}}$ is group-like for $\lambda \in \Omega^{+}$with $\ell(\lambda) \leq N$. Taking the dual, we see that $D_{k} T=D_{k}{ }^{t} T$ is a subalgebra of the Schur algebra $D_{k} E$, and $\left\{t^{*(\lambda)}=t_{1}^{*\left(\lambda_{1}\right)} \cdots t_{N}^{*\left(\lambda_{N}\right)} \mid \lambda \in \Omega_{k}^{+}\right.$, $\ell(\lambda) \leq N\}$ is a set of mutually orthogonal primitive idempotents of $D_{k} T$. It follows that $1=\sum_{\lambda} t^{*(\lambda)}$, and that $D_{k} T \simeq \prod_{\lambda} t^{*(\lambda)}\left(D_{k} T\right) t^{*(\lambda)} \simeq \prod_{\lambda} R$.

Definition 7.7. Let $M \in \mathscr{P}$ and $\lambda \in \Omega_{k}^{+}$with $\ell(\lambda) \leq N$. We call the $R$-module $t^{*(\lambda)} M_{k}$ the weight $\lambda$ component of $M$, and denote it by $M_{\lambda}$.

Clearly, $M_{\lambda}$ is a finitely generated projective $R$-module. By [6, p. 375], we have

$$
M_{\lambda}=t^{*(\lambda)} M_{k} \simeq \operatorname{Hom}_{D_{k} T}\left(\left(D_{k} T\right) t^{*(\lambda)}, M\right) \simeq \operatorname{Hom}_{D_{k} E}\left(\left(D_{k} E\right) t^{*(\lambda)}, M\right),
$$

so that ( $)_{\lambda}$ is an exact functor $\left(\left(D_{k} E\right) t^{*(\lambda)}\right.$ is projective). Since $1=\sum_{\lambda} t^{*(\lambda)}$, we have $M=\oplus_{\lambda} M_{\lambda}$. It is easy to see that $M_{\lambda}=\left\{m \in M \mid \omega(m)=\mathrm{m} \otimes t_{\lambda}\right\}$, where $\omega$ is the coaction as an $S T$-comodule. For a base change $R \rightarrow R^{\prime}$, we have $\left(R^{\prime} \otimes_{R} M\right)_{\lambda} \simeq R^{\prime} \otimes_{R} M_{\lambda}$. If the prime spectrum Spec $R$ is connected, then $\operatorname{dim}_{K}\left(K \otimes_{R} M\right)_{\lambda}$ does not depend on the field $K$ or the map $R \rightarrow K$.

LEMMA 7.8. Let $M \in \mathscr{P}$ and $\lambda \in \Omega_{k}^{+}$with $\ell(\lambda) \leq N$. Then we have an isomorphism of $R$-modules $\left(M^{\circ}\right)_{\lambda} \simeq M_{\lambda}^{*}$.

Proof. We may replace $M$ by $M_{k}$. Clearly, we have $M^{*} t^{*(\lambda)} \simeq\left(M_{\lambda}\right)^{*}$. On the other hand, the restriction of $\operatorname{tr}^{\dagger}$ to $D T$ is the identity map.

Proposition 7.9. For $k \geq 0$ and $\lambda \in \Omega_{k}^{+}$with $\ell(\lambda) \leq N$, we have:

(1) $\left(D_{k} E\right) t^{*(\lambda)} \simeq D_{\lambda} V$ as $S E^{\vee}$-comodules.

(2) $D_{\lambda} V$ is projective, and is generated by $c(\lambda):=u_{1}^{\left(\lambda_{1}\right)} \otimes \cdots \otimes u_{N}^{\left(\lambda_{N}\right)}$ as a left $D_{k} E$-module.

Proof. First note that the identification $S E^{\vee} \simeq S^{t} E$ maps a monomial $M$ to $M$. Hence, the identification $D_{k} E \simeq D_{k} E^{-1}$ maps $t^{*(\lambda)}$ to $t^{*(\lambda)}$. For $i \in[1, N]$, we define $\varphi_{i}: V \rightarrow E=V^{*} \otimes V^{*}$ by $\varphi_{i}\left(u_{j}\right)=u_{j} \otimes v_{i}$. It is clear that $\varphi_{i} \in \mathscr{Y} \mathscr{B}_{R}(V, E)$, and that $\varphi_{i}$ is a homomorphism of left $\operatorname{End}(V)=E$-module. Hence, the composite map

$$
\varphi_{\lambda}: D_{\lambda} V=D_{\lambda_{1}} V \otimes \cdots \otimes D_{\lambda_{N}} V \stackrel{D_{\lambda_{1}} \varphi_{1} \otimes \cdots \otimes D_{\lambda_{N}} \varphi_{N}}{\longrightarrow} D_{\lambda_{1}} E \otimes \cdots \otimes D_{\lambda_{N}} E \stackrel{m}{\longrightarrow} D_{k} E
$$

is a homomorphism of left $D_{k} E$-modules. Observe that $\varphi_{\lambda}(c(\lambda))=t^{*(\lambda)}$ (cf. Lemma 6.22). The sum $\sum_{\lambda} \varphi_{\lambda}: \oplus_{\lambda} D_{\lambda} V \rightarrow D_{k} E$ is surjective, since $\sum_{\lambda} \varphi_{\lambda}(c(\lambda))=1$. On the other hand, we have $\operatorname{rank}_{R}\left(\oplus_{\lambda} D_{\lambda} V\right)=\operatorname{rank}_{R}\left(D_{k} E\right)$ (since the rank in question is stable under base change, we may assume that $R=C$ and $q=1$. In this case, see e.g. [2]). It follows that $\sum_{\lambda} \varphi_{\lambda}$ is an isomorphism. Since $\sum_{\lambda} \varphi_{\lambda}$ is injective, and $\operatorname{Im} \varphi_{\lambda} \supset\left(D_{k} E\right) t^{*(\lambda)}$ for each $\lambda$, we have $\operatorname{Im} \varphi_{\lambda}=\left(D_{k} E\right) t^{*(\lambda)}$, and $\varphi_{\lambda}: D_{\lambda} V \rightarrow\left(D_{k} E\right) t^{*(\lambda)}$ is an isomorphism. Hence, we have (1). Now (2) is obvious. 
COROLlary 7.10. Let $\lambda \in \Omega_{k}^{+}$and $\ell(\lambda) \leq N$. For $M \in \mathscr{P}$, we have an isomorphism $\Psi_{\lambda}: M_{\lambda} \neg \mathscr{P}\left(D_{\lambda}, M\right)$ given by $\left(\Psi_{\lambda}(a)\right)(c(\lambda))=$ a for $a \in M_{\lambda}$.

LEMMA 7.11. Let $\lambda \in \Omega^{+}$with $\ell(\lambda) \leq N$. For any $\sigma \in \mathfrak{\Xi}_{N}$, we have an isomorphism of $S E^{\vee}$-comodules $D_{\lambda} V \simeq D_{\sigma \lambda} V$, where $\sigma \lambda=\left(\lambda_{\sigma^{-1} 1}, \cdots, \lambda_{\sigma^{-1} N}\right)$. In particular, we have $D_{\lambda} V \simeq D_{(\tilde{\lambda})^{\sim}} V$. For $M \in \mathrm{ob} \mathscr{P}$, we have $M_{\lambda} \simeq M_{\sigma \lambda}$.

Proof. The $S E^{\vee}$-isomorphism $\psi_{D V}(\sigma):(D V)^{\otimes N} \rightarrow(D V)^{\otimes N}$ maps $D_{\lambda} V$ onto $D_{\sigma \lambda} V$. The last assertion follows from Corollary 7.10.

Let $\lambda$ and $\mu$ be elements in $\Omega^{+}$such that $\ell(\lambda), \ell(\mu) \leq N$. We say that $\mu \leqq \lambda$ when $\lambda-\mu$ is a non-negative linear combination of $\alpha_{i}=\varepsilon_{i}-\varepsilon_{i+1}(i \in[1, N-1])$. It is easy to see that $\mu \leqq \lambda$ implies $|\mu|=|\lambda|$ and $\mu \leq \lambda$. For $\lambda \in \Omega^{+}$with $\ell(\lambda) \leq N$, we have $\lambda \leqq \tilde{\lambda}^{\sim}$. For $\mu \in \Omega^{-}$with $\ell(\mu) \leq N$ and $\lambda \in S_{\square}(\mu)$, we have $\mu \prec \lambda$ by the definition of $S_{\square}(\lambda)$ (see $\S 6$ ).

THEOREM 7.12. Let $k \geq 0$, and $\lambda \in \Omega_{k}^{-}$with $\ell(\lambda) \leq N$. Then we have:

(1) $\mu \in \Omega^{-}, \ell(\mu) \leq N,\left(K_{\lambda} V\right)_{\mu} \neq 0 \Rightarrow \mu \leqq \lambda$.

(2) $\left(K_{\lambda} V\right)_{\lambda} \simeq R$.

(3) $K_{\lambda} V$ is generated by $\left(K_{\lambda} V\right)_{\lambda}$ as a $D_{k} E$-module.

Let $M$ be a $D_{k} E$-module which satisfies:

(1') $\mu \in \Omega_{k}^{-}, \ell(\mu) \leq N, \mu>\lambda \Rightarrow M_{\mu}=0$.

(3') $M$ is generated by some $a \in M_{\lambda}$.

Then $M$ is a homomorphic image of $K_{\lambda} V$. Moreover, we have:

(1") $\quad \mu \in \Omega^{-}, \ell(\mu) \leq N, M_{\mu} \neq 0 \Rightarrow \mu \leqq \lambda$.

(2') $\operatorname{Hom}_{D_{k} E}\left(K_{\lambda} V, M\right) \simeq M_{\lambda} \simeq R a$.

Conversely, any homomorphic image $M$ of $K_{\lambda} V$ satisfies (1') and (3').

Proof. By Proposition $7.9(2), K_{\lambda} V$ is generated by $d_{\lambda}^{\prime}(V)(c(\lambda)) \in\left(K_{\lambda} V\right)_{\lambda}$. So we have (3). By Corollary 6.24, $\operatorname{rank}_{R}\left(K_{\lambda} V\right)_{\mu}$ does not depend on $R$ nor $q$. Parts (1) and (2) are well-known in the case $R=C$ and $q=1$ (see [4]). So the general case immediately follows, since any rank one projective module over Laurent polynomial ring $Z\left[Q, Q^{-1}\right]$ is free. Now the last assertion is obvious, since ( $)_{\mu}$ is an exact functor for any $\mu$. Let $M$ be a $D_{k} E$-module which satisfies $\left(1^{\prime}\right)$ and $\left(3^{\prime}\right)$. By $\left(3^{\prime}\right)$ and Corollary 7.10, we have a surjective map $\Psi_{\lambda}(a): D_{\lambda} V \rightarrow M$. By Theorem 6.23 , we have an exact sequence

$$
0 \rightarrow \operatorname{Hom}_{D_{k} E}\left(K_{\lambda} V, M\right) \rightarrow \operatorname{Hom}_{D_{k} E}\left(D_{\lambda} V, M\right) \rightarrow \oplus_{\mu \in S_{\square}(\lambda)} \operatorname{Hom}_{D_{k} E}\left(D_{\mu} V, M\right) .
$$

For $\mu \in S_{\square}(\lambda)$, we have $\operatorname{Hom}_{D_{k} E}\left(D_{\mu} V, M\right) \simeq M_{\mu} \simeq M_{\tilde{\mu}^{\sim}}$ by Lemma 7.11. Since $\tilde{\mu}^{\sim} \geqq \mu>\lambda$ and $\tilde{\mu}^{\sim} \in \Omega_{k}^{-}$, we have $M_{\tilde{\mu}^{\sim}}=0$ by $\left(1^{\prime}\right)$. It follows that $\operatorname{Hom}_{D_{k} E}\left(d_{\lambda}^{\prime}(V), M\right): \operatorname{Hom}_{D_{k} E}\left(K_{\lambda} V\right.$, $M) \rightarrow \operatorname{Hom}_{D_{k} E}\left(D_{\lambda} V, M\right) \simeq M_{\lambda}$ is an isomorphism. In particular, we have an induced surjective map $\overline{\Psi_{\lambda}(a)}: K_{\lambda} V \rightarrow M$. Now $\left(1^{\prime \prime}\right)$ and $\left(2^{\prime}\right)$ are obvious.

REMARK 7.13. Since $D V$ and $D V^{-1}$ are isomorphic as $S E^{\vee}$-equivariant YB coalgebras, we have $D_{\lambda} V \simeq D_{\lambda} V^{-1}$ for $\lambda \in \Omega^{+}$. So it is easy to see that $K_{\lambda} V^{-1}$ satisfies 
(1') and (3') in the theorem for $\lambda \in \Omega^{-}$with $\ell(\lambda) \leq N$. We have a surjective map $K_{\lambda} V \rightarrow K_{\lambda} V^{-1}$. This map is an isomorphism, since $K_{\lambda} V$ and $K_{\lambda} V^{-1}$ have the same rank. Since $\bigwedge V$ and $\bigwedge V^{-1}$ are isomorphic as $S E^{\vee}$-equivariant $\mathrm{YB}$ algebras, the image $K_{\lambda}^{\prime} V$ of the composite map

$$
d_{\lambda}^{\prime \prime}(V): D_{\lambda} V \stackrel{\Delta \otimes \cdots \otimes \Delta}{\longrightarrow} T_{\lambda} V \stackrel{\left(-\gamma_{V}^{-1}\right)\left(\chi_{\lambda}\right)}{\longrightarrow} T_{\tilde{\lambda}} V \stackrel{m \otimes \cdots \otimes m}{\longrightarrow} \bigwedge_{\tilde{\lambda}} V
$$

is isomorphic to $K_{\lambda} V^{-1}$. Hence, we have $K_{\lambda}^{\prime} V \simeq K_{\lambda} V$. Taking the contravariant dual, we have $L_{\tilde{\lambda}} V \simeq L_{\tilde{\lambda}} V^{-1} \simeq\left(K_{\lambda}^{\prime} V\right)^{\circ}$.

As we will see in the next section, the study of $S E^{\vee}$-comodules is closely related to the representations of the Iwahori Hecke algebra. The Iwahori Hecke algebra $\mathfrak{H}_{k}$ is defined to be the quotient algebra

$$
\mathfrak{G}_{k} \simeq R\left[\mathfrak{B}_{k}\right] /\left(\left(1-b_{i}\right)\left(1+q^{-2} b_{i}\right) \mid i=1, \ldots, k-1\right) .
$$

We set $t=q^{-2}$. By Iwahori's theorem, $h(\sigma):=t^{\ell(\sigma)} \bar{b}_{i_{1}} \cdots \bar{b}_{i_{\ell(\sigma)}}$ is independent of the choice of the reduced expression $\sigma=\sigma_{i_{1}} \cdots \sigma_{i_{\ell(\sigma)}}$ of $\sigma \in \mathfrak{S}_{k}$, where $\bar{b}_{j}(1 \leq j<k)$ is the image of $b_{j}$ in $\mathfrak{S}_{k}$. It is known and easily shown that $\mathfrak{S}_{k}$ is $R$-free of rank $k$ !, and $\left\{h(\sigma) \mid \sigma \in \mathfrak{S}_{k}\right\}$ is a free basis of $\mathfrak{H}_{k}$. By (1.4), the representation of $R\left[\mathfrak{B}_{k}\right]$ on $V^{\otimes k}$ given by (1.8) yields the representation $B: \mathfrak{H}_{k} \rightarrow \operatorname{End}_{R} V^{\otimes k}$ given by $B\left(\bar{b}_{i}\right)=\left(\beta_{V}\right)_{i}(1 \leq i<k)$. Since $D_{k} E=$ $\operatorname{End}_{\mathfrak{G}_{k}} V^{\otimes k}$, we have $\operatorname{Im} B \subset \operatorname{End}_{D_{k}} V^{\otimes k}$. Note that $B(h(\sigma))=\left(-\gamma_{V}\right)(\sigma)$ for $\sigma \in \mathfrak{S}_{k}$.

LEMmA 7.14. Assume that $N \geq k$. Then, $B: \mathfrak{S}_{k} \rightarrow \operatorname{End}_{D_{k} E} V^{\otimes k}$ is an isomorphism.

ProOF. Since $V^{\otimes k}=D_{\left(1^{k}\right)} V$, we have an isomorphism $\Psi_{\left(1^{k}\right)}:\left(V^{\otimes k}\right)_{\left(1^{k}\right)} \neg \operatorname{End}_{D_{k} E} V^{\otimes k}$ by Corollary 7.10. Under this identification, $B: \mathfrak{H}_{k} \rightarrow V_{\left(1^{k}\right)}^{\otimes k}$ is given by $B(h(\sigma))=$ $\left(-\gamma_{V}\right)(\sigma)\left(u_{1} \otimes \cdots \otimes u_{k}\right)$. By $(5.4)$, we have $\left(-\gamma_{V}\right)(\sigma)\left(u_{1} \otimes \cdots \otimes u_{k}\right)=q^{-\ell(\sigma)}\left(u_{\sigma^{-1} 1_{1}} \otimes\right.$ $\left.\cdots \otimes u_{\sigma^{-1}{ }_{k}}\right)$. Hence, $B$ is bijective.

8. Polynomial representations of $G L_{q}$ over a field. In this section, we work on a base field $K$, instead of $R$. Throughout this section, $V=\left(V, \beta_{V}, \gamma_{V}\right)$ and $E=\left(E, \beta_{E}, \gamma_{E}\right)$ are as in $§ 7$. A polynomial representation of $G L_{q}(N, K)$ is a finite-dimensional right $S E^{\vee}$-comodule by definition. This section is devoted to studying polynomial representations of $G L_{q}(N, K)$ over a field $K$. Note that the categories cff- $S_{k} E$ and $\mathscr{P}$ (§7) are abelian, and that the contravariant dual ( $)^{\circ}$ is exact.

Definition 8.1. For a polynomial representation $M$ of $G L_{q}(N, K)$, we define a polynomial

$$
\chi(M)=\sum_{\lambda \in \Omega^{+}, \ell(\lambda) \leq N}\left(\operatorname{dim}_{K} M_{\lambda}\right) t_{1}^{\lambda_{1}} \cdots t_{N}^{\lambda_{N}} \in Z\left[t_{1}, \ldots, t_{N}\right]
$$

in the variables $t_{1}, \ldots, t_{N}$, and call it the formal character of $M$. 
By Lemma $7.11, \chi(M)$ is a symmetric polynomial in $t_{1}, \ldots, t_{N}$. For polynomial representations $M$ and $N$ of $G L_{q}(N, K)$ and $\lambda \in \Omega^{+}$with $\ell(\lambda) \leq N$, we have $(M \otimes N)_{\lambda} \simeq \bigoplus_{\mu+\mu^{\prime}=\lambda} M_{\mu} \otimes N_{\mu^{\prime}}$. Since ( $)_{\lambda}$ is an exact functor for $\lambda \in \Omega^{+}$with $\ell(\lambda) \leq N$, the formal character $\chi$ is a homomorphism from the Grothendiek ring POL of polynomial representations of $G L_{q}(N, K)$ to the ring $Z\left[t_{1}, \ldots, t_{N}\right]^{\Im_{k}}$ of symmetric polynomials. If $\tilde{M}$ is an $S E^{\vee}$-comodule over the Laurent polynomial ring $Z\left[Q, Q^{-1}\right]$ such that it is $Z\left[Q, Q^{-1}\right]$-free of finite rank, then $\chi\left(K \otimes_{\mathbf{z}\left[Q, Q^{-1}\right]} \tilde{M}\right)$ does not depend on the field $K$ nor the value of $q$ by the remark before Lemma 7.8. Hence, for $k \geq 0$ and $\lambda \in \Omega^{-}$with $\ell(\lambda) \leq N$, we obtain the following formulas:

$$
\begin{aligned}
& \chi\left(\bigwedge_{k} V\right)=e_{k}:=\sum_{1 \leq i_{1}<\cdots<i_{k} \leq N} t_{i_{1}} \cdots t_{i_{k}} . \\
& \chi\left(S_{k} V\right)=h_{k}:=\sum_{M} M, \text { where } M \text { runs through the monomials of degree } k . \\
& \chi\left(K_{\lambda} V\right)=s_{\lambda}:=\operatorname{det}\left(h_{\lambda_{i}-i+j}\right)_{1 \leq i, j \leq N}=\operatorname{det}\left(e_{\tilde{\lambda}_{i}-i+j}\right)_{1 \leq i, j \leq N} .
\end{aligned}
$$

Lemma 8.2. For a polynomial representation $M$ of $G L_{q}(N, K)$, we have $\chi\left(M^{\circ}\right)=\chi(M)$.

This follows immediately from Lemma 7.8 .

In the rest of this section, we fix $k \geq 0$, and set $A=D_{k} E$. We denote the Jacobson radical of $A$ by $J$. For $M \in A$-mff, $(M / J M)^{\circ}$ is the unique maximal semisimple submodule of $M^{\circ}$. Namely, we have $(M / J M)^{\circ} \simeq$ Soc $M^{\circ}$, where Soc denotes the socle.

LEMMA 8.3. Let $\lambda \in \Omega_{k}^{-}$and $\ell(\lambda) \leq N$. Then, $K_{\lambda} V / J\left(K_{\lambda} V\right) \simeq\left(\operatorname{Soc}\left(L_{\tilde{\lambda}} V\right)\right)^{\circ}$ is simple (irreducible). In particular, $K_{\lambda} V$ and $L_{\tilde{\lambda}} V$ are indecomposable.

Proof. Let $M$ be a proper submodule of $K_{\lambda} V$. Then, we have $M_{\lambda}=0$ by (2) and (3) of Theorem 7.12. We define $\left(K_{\lambda} V\right)_{\max }$ to be the sum of all proper submodules of $K_{\lambda} V$. We have $\left(K_{\lambda} V\right)_{\max } \neq K_{\lambda} V$, since $\left(\left(K_{\lambda} V\right)_{\max }\right)_{\lambda}=0$. It follows that $\left(K_{\lambda} V\right)_{\max }$ is the unique maximal proper submodule of $K_{\lambda} V$. Hence, we have $\left(K_{\lambda} V\right)_{\max }=J\left(K_{\lambda} V\right)$, and $K_{\lambda} V / J\left(K_{\lambda} V\right)$ is simple. By Corollary 7.5, we have $\left(\operatorname{Soc}\left(L_{\tilde{\lambda}} V\right)\right)^{\circ} \simeq K_{\lambda} V / J\left(K_{\lambda} V\right)$.

For $\lambda \in \Omega_{k}^{-}$with $\ell(\lambda) \leq N$, we denote by $F_{\lambda}$ the simple module $K_{\lambda} V / J\left(K_{\lambda} V\right)$.

Proposition 8.4. Each simple A-module is isomorphic to $F_{\lambda}$ for exactly one $\lambda \in \Omega_{k}^{-}$ such that $\ell(\lambda) \leq N$.

Proof. In this proof, we use the lexicographic order $\leq$ of the set $\left\{\lambda \in \Omega_{k}^{-} \mid \ell(\lambda) \leq N\right\}$. For $0 \neq M \in A$-mff, we define

$$
h(M): \neq \max \left\{\lambda \in \Omega_{k}^{-} \mid \ell(\lambda) \leq N, M_{\lambda} \neq 0\right\} .
$$

Since $0 \neq M=\oplus_{\lambda} M_{\lambda}$ and $M_{\lambda} \simeq M_{\tilde{\lambda} \sim}$, the partition $h(M)$ is well-defined. Since $F_{\lambda}$ is a non-zero homomorphic image of $K_{\lambda} V$, we have $h\left(F_{\lambda}\right)=\lambda$ by Theorem 7.12. Hence, 
$F_{\lambda} \nsucceq F_{\mu}$ for $\mu \neq \lambda$. Let $M$ be a simple $A$-module. By the definition of $h(M)$, the condition $\left(1^{\prime}\right)$ of Theorem 7.12 is satisfied for $\lambda=h(M)$. Since $M$ is simple, the condition ( $\left.3^{\prime}\right)$ of Theorem 7.12 is satisfied for any non-zero vector $a \in M_{h(M)}$. Hence, $M$ is a homomorphic image of $K_{h(M)}$, and we have $M \simeq F_{h(M)}$.

COROLlaRY 8.5. The formal character $\chi: \mathrm{POL} \rightarrow Z\left[t_{1}, \ldots, t_{N}\right]^{\varsigma_{N}}$ is a ring isomorphism, where POL is the Grothendieck ring of polynomial representations of $G L_{q}(N, K)$.

Proof. It suffices to show that $\chi:[A-\mathrm{mff}] \rightarrow \operatorname{Sym}(k)$ is a bijection, where $[A-\mathrm{mff}]$ denotes the Grothendieck group of $A$-mff and $\operatorname{Sym}(k)$ is the module of symmetric polynomials of degree $k$ in $t_{1}, \ldots, t_{N}$. By [25], $\operatorname{Sym}(k)$ is a $Z$-free module with a free basis $\left\{s_{\lambda} \mid \lambda \in \Omega_{k}^{-}, \ell(\lambda) \leq N\right\}$. By $(8.4), \chi:[A-\mathrm{mff}] \rightarrow \operatorname{Sym}(k)$ is surjective. By the proposition, we have $\operatorname{rank}[A-\mathrm{mff}]=\operatorname{rank} \operatorname{Sym}(k)$.

COROllary 8.6. Let $M \in A$-mff. Then, $M$ and $M^{\circ}$ have the same composition factors. In particular, We have $F_{\lambda} \simeq F_{\lambda}^{\circ} \simeq \operatorname{Soc}\left(L_{\lambda} V\right)$ for $\lambda \in \Omega_{k}^{-}$with $\ell(\lambda) \leq N$.

This follows immediately from Lemma 8.2 and Corollary 8.5.

LEMMA 8.7. Let $\lambda \in \Omega_{k}^{-}$with $\ell(\lambda) \leq N$. Then, the following are equivalent:

(1) $K_{\lambda} V$ is simple.

(2) $L_{\tilde{\lambda}} V$ is simple.

(3) $K_{\lambda} V \simeq L_{\tilde{\lambda}} V$.

Proof. $K_{\lambda} V \simeq F_{\lambda} \Leftrightarrow\left(K_{\lambda} V\right)^{\circ} \simeq F_{\lambda}^{\circ} \Leftrightarrow L_{\tilde{\lambda}} V \simeq F_{\lambda}^{\circ}$. Hence, we have $(1) \Leftrightarrow(2)$. By Corollary 8.6, (1) (or (2)) also implies (3). We shall show that (3) $\Rightarrow(2)$. By Theorem 7.12, $L_{\tilde{\lambda}} V$ is generated by $\left(L_{\tilde{\lambda}} V\right)_{\lambda}$. Since both $\left(L_{\tilde{\lambda}} V\right)_{\lambda}$ and $\left(F_{\lambda}\right)_{\lambda}$ are one-dimensional, $L_{\tilde{\lambda}} V$ is generated by its simple socle $F_{\lambda}$. Hence, we have $L_{\tilde{\lambda}} V \simeq F_{\lambda}$.

LEMma 8.8. Let $\lambda \in \Omega_{k}^{-}$and $\ell(\lambda) \leq N$. Then, $F_{\lambda}$ is absolutely irreducible.

Proof. Since $0 \neq \operatorname{End}_{A} F_{\lambda} \subset \operatorname{Hom}_{A}\left(K_{\lambda}, F_{\lambda}\right) \simeq K$ by Theorem 7.12, $\operatorname{End}_{A} F_{\lambda}$ is one-dimensional. The lemma follows from $[6,(29.13)]$.

The rest of this sections is devoted to the proof of the following theorem.

THEOREM 8.9. Let $K$ be a field, $\left(V, \beta_{V}\right)$ be Jimbo's YB pair of type $A_{N-1}^{(1)}$ defined by $q \in K^{\times}$and (1.3), and let $\lambda$ be a partition with $\ell(\lambda) \leq N$. If the product $\prod_{(i, j) \in \Delta_{\lambda}} h_{q}(i, j)$ of (q-analogues of) hook lengths is non-zero, then the Weyl module $K_{\lambda} V$ is simple (irreducible) and projective, where $h_{q}(i, j)=\left[\lambda_{i}+\tilde{\lambda}_{j}-i-j+1\right]_{q-2}$.

To prove the theorem, we use Gyoja's $q$-analogues of Young symmetrizers [14]. For this purpose, let us clarify the relationship between the notation in [14] and that of ours. Our size $k$ of the Iwahori Hecke algebra, is denoted by $n$ in [14]. The notation $h(\sigma)$ agrees, while $q$ in [14] corresponds to our $t=q^{-2}$. To avoid confusion, we will 
stick to our notation $k$, (our) $q$ and $t=q^{-2}$. For $\lambda \in \Omega_{k}^{-}$, our $\Im_{\lambda}, \Im_{\tilde{\lambda}}, \alpha_{r, \lambda}^{-1}, \alpha_{c, \lambda}^{-1}, \chi_{\lambda}$ and $\chi_{\tilde{\lambda}}=\chi_{\lambda}^{-1}$ correspond to $W_{+}(\lambda), W_{-}(\lambda), T_{+}(\lambda), T_{-}(\lambda),[-,+]$ and $[+,-]$ in [14], respectively. Hence, we have

$$
\begin{aligned}
& B e_{+}=\sum_{\sigma \in \mathfrak{G}_{\lambda}} B h(\sigma)=\sum_{\sigma \in \mathfrak{G}_{\lambda}}\left(-\gamma_{V}\right)(\sigma), \\
& B e_{-}=\sum_{\sigma \in \mathfrak{G}_{\lambda}}(-t)^{-\ell(\sigma)} B h(\sigma)=\sum_{\sigma \in \mathfrak{G}_{\lambda}}\left(-\beta_{V}\right)(\sigma),
\end{aligned}
$$

where $e_{+}=e_{+}(\lambda)$ and $e_{-}=e_{-}(\lambda)$ are those in [14], and $B$ is as defined in $\S 7$.

Proof OF Theorem 8.9. We set $k=|\lambda|$. It suffices to show that $K_{\lambda}^{\prime} V$ (see Remark 7.13) is simple and projective. We define the map $\Phi_{\lambda}: S_{\lambda} V \rightarrow D_{\lambda} V$ to be the restriction of $T_{N} \Phi:(S V)^{\otimes N} \rightarrow(D V)^{\otimes N}$ to $S_{\lambda} V$, where $\Phi=\Phi^{S V}$ is the map which appears in Proposition 5.2. By the remark below Lemma 5.8, $\Phi_{\lambda}$ is a homomorphism of $S E^{\vee}$-comodules. We denote by $\kappa_{\lambda}(V)$ the composite map

$$
K_{\lambda}^{\prime} V \smile \bigwedge_{\tilde{\lambda}} V \stackrel{d_{\lambda}(V)}{\longrightarrow} L_{\tilde{\lambda}} V \smile S_{\lambda} V \stackrel{\Phi_{\lambda}}{\longrightarrow} D_{\lambda} V \stackrel{d_{\lambda}^{\prime \prime}(V)}{\longrightarrow} K_{\lambda}^{\prime} V,
$$

where $d_{\lambda}^{\prime \prime}(V)$ is the map defined in Remark 7.13. The map $\kappa_{\lambda}(V)$ is defined over the Laurent polynomial ring $Z\left[Q, Q^{-1}\right]$, and is a multiplication by a scalar, say $c$, by Theorem 7.12. We claim that $c \in Z\left[Q, Q^{-1}\right]$ is expressed as $c=Q^{*} \prod_{(i, j) \in \Delta_{\lambda}} h_{Q}(i, j)$. Assume that the claim is true. Then, $\kappa_{\lambda}(V)=q^{*} \prod h_{q}(i, j) \neq 0$ is an isomorphism over $K$. Since the isomorphism $\kappa_{\lambda}(V)$ factors through $L_{\tilde{\lambda}} V$ and $\operatorname{dim}_{K} K_{\lambda}^{\prime} V=\operatorname{dim}_{K} L_{\tilde{\lambda}} V$, we have $K_{\lambda}^{\prime} V \simeq L_{\tilde{\lambda}} V$. By Lemma 8.7, $K_{\lambda}^{\prime} V$ is simple. Since the scalar multiplication $\kappa_{\lambda}(V)$ factors through $D_{\lambda} V$, the epimorphism $d_{\lambda}^{\prime \prime}(V): D_{\lambda} V \rightarrow K_{\lambda}^{\prime} V$ splits. Since $D_{\lambda} V$ is projective by Proposition 7.9, (2), the direct summand $K_{\lambda}^{\prime} V$ is also projective, and the proof is complete. To prove the claim, we may extend the base ring and work over the base field $K=C(Q)$. We still use the notation $t=Q^{-2}$. We denote the maps $m \otimes \cdots \otimes m: V^{\otimes k} \simeq T_{\lambda} V \rightarrow D_{\lambda} V$, $\Delta \otimes \cdots \otimes \Delta: D_{\lambda} V \rightarrow T_{\lambda} V \simeq V^{\otimes k}, m \otimes \cdots \otimes m: V^{\otimes k} \simeq T_{\tilde{\lambda}} V \rightarrow \bigwedge_{\tilde{\lambda}} V, \Delta \otimes \cdots \otimes \Delta: \bigwedge_{\tilde{\lambda}} V \rightarrow$ $T_{\tilde{\lambda}} V \simeq V^{\otimes k}, m \otimes \cdots \otimes m: V^{\otimes k} \simeq T_{\lambda} V \rightarrow S_{\lambda} V$ and $\Delta \otimes \cdots \otimes \Delta: S_{\lambda} V \rightarrow T_{\lambda} V \simeq V^{\otimes k}$ by $m_{D}$, $\Delta_{D}, m_{\wedge}, \Delta_{\wedge}, m_{S}$ and $\Delta_{S}$, respectively. Since $\Phi$ is a homomorphism of YB bialgebras, it holds that $\Phi_{\lambda} \circ m_{S}=m_{D}$ and $\Delta_{D} \circ \Phi_{\lambda}=\Delta_{S}\left(\Phi_{\left(1^{k}\right)}\right.$ is identified with $\mathrm{id}_{V} \otimes_{k}$ and omitted). By (5.1) and (8.7), we have $B e_{-}=\Delta \wedge^{\circ} m_{\wedge}$. By (5.2) and (8.6), we have $B e_{+}=\Delta_{S} \circ m_{S}=$ $\Delta_{D}^{\circ} \Phi_{\lambda} \circ m_{S}=\Delta_{D} \circ m_{D}$. Now consider the map

$$
V^{\otimes k} \stackrel{m_{D}}{\longrightarrow} D_{\lambda} V \stackrel{d_{\lambda}^{\prime \prime}}{\longrightarrow} K_{\lambda}^{\prime} V \stackrel{\kappa_{\lambda}}{\longrightarrow} K_{\lambda}^{\prime} V \smile \bigwedge \bigwedge_{\lambda} V \stackrel{\Delta_{\Lambda}}{\longrightarrow} V^{\otimes k} \stackrel{h_{-}}{\longrightarrow} V^{\otimes k},
$$

where $h_{-}=h([-,+])$. Since the diagram 


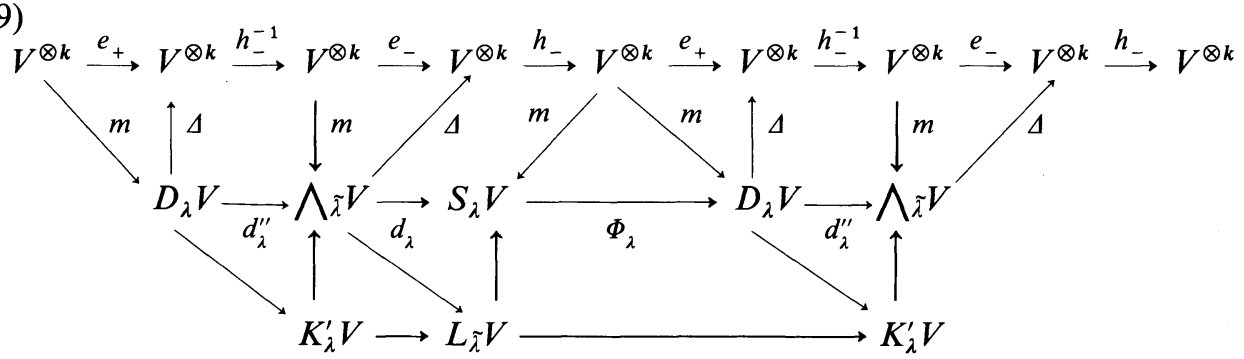

is commutative and the bottom row map is $\kappa_{\lambda}(V)$, the map (8.8) is $B\left(h_{-} e_{-} h_{-}^{-1} e_{+}\right)^{2}$. On the other hand, we have $\kappa_{\lambda}(V)=c$. So the map (8.8) equals $c B\left(h_{-} e_{-} h_{-}^{-1} e_{+}\right)$by the same diagram. Hence, we have

$$
B\left(h_{-} e_{-} h_{-}^{-1} e_{+}\right)^{2}=c B\left(h_{-} e_{-} h_{-}^{-1} e_{+}\right) .
$$

Since $K=C(Q)$, we see that $\Delta: S_{i}{ }^{t} V \rightarrow T_{i}{ }^{t} V(i \geq 0)$ is injective by Lemma 5.4. It follows that $m_{D}$ is surjective. Again by (8.9), we have $\left(h_{-} e_{-} h_{-}^{-1} e_{+}\right) V^{\otimes k} \simeq K_{\lambda}^{\prime} V \neq 0$. Hence, $c$ is uniquely determined by (8.10).

It is now clear that the scalar $c$ is nothing but $c\left(T_{+}\right)$in [14]. Here we recall the value of $c[14,(3.9)]$.

$$
\left.c=\frac{\prod_{i=1}^{m} \sum_{l=1}^{\lambda_{i}+m-i}\left(t^{l}-1\right)}{\prod_{1 \leq i<j \leq m}\left(t^{\lambda_{i}+m-i}-t^{\lambda_{j}+m-j}\right)} t^{m} \begin{array}{c}
m \\
3
\end{array}\right) \cdot(t-1)^{-k}
$$

where $m$ is any integer such that $m \geq N$. By [25, p. 9], we have

$$
c=\frac{\prod_{i} \prod_{l=1}^{\mu_{i}}\left(t^{l}-1\right)}{\prod_{i<j}\left(t^{\mu_{i}-\mu_{j}}-1\right)} t^{*}(t-1)^{-k}=t^{*} \prod_{(i, j) \in \Delta_{\lambda}} \frac{t^{h_{1}(i, j)}-1}{t-1}=t^{*} \prod_{(i, j) \in \Delta_{\lambda}} h_{Q}(i, j),
$$

as desired, where $\mu=\left(\mu_{1}, \ldots, \mu_{m}\right)$ is given by $\mu_{i}=\lambda_{i}+m-i$ for $i \in[1, m]$.

COROllary 8.10. Assume that $[a]_{t} \neq 0$ for $a \in[1, k]$. Then the Schur algebra $A=\operatorname{End}_{\mathfrak{G}_{k}} V^{\otimes k}$ is semisimple, and $\left\{K_{\lambda} \mid \lambda \in \Omega_{k}^{-}, \ell(\lambda) \leq N\right\}$ is a complete set of non-isomorphic simple A-modules. In this case, the Iwahori Hecke algebra $\mathfrak{G}_{k}$ is also semisimple.

Proof. By the theorem, $K_{\lambda} V$ is simple and projective for any $\lambda \in \Omega_{k}^{-}$with $\ell(\lambda) \leq N$. By Proposition 8.4, $\left\{K_{\lambda} \mid \lambda \in \Omega_{k}^{-}, \ell(\lambda) \leq N\right\}$ is a complete set of non-isomorphic simple $A$-modules. Since each simple module is projective, $A=\operatorname{End}_{\mathfrak{S}_{k}} V^{\otimes k}$ is semisimple. Applying this for $N \geq k$, we see that $\mathfrak{H}_{k}=\operatorname{End}_{A} V^{\otimes k}$ is also semisimple, by the double centralizer theorem. 
Note. Gyoja and Uno [15] proved the semisimplicity of $\mathfrak{H}_{k}$ under the same assumption as in the corollary above (and some extra condition on the base field, which can be dropped easily). The semisimplicity of $A$ also follows from their result using the double centralizer theorem.

COROLlaRY 8.11. In the situation of Corollary 8.10, $A=\operatorname{End}_{\mathfrak{F}_{k}} V^{\otimes k}$ is isomorphic to $\prod_{\lambda \in \Omega_{\bar{k}}, \ell(\lambda) \leq N} \operatorname{End}_{K}\left(K_{\lambda} V\right)$ as a K-algebra. We have an irreducible decomposition

$$
A \simeq S_{k} E^{\vee} \simeq \underset{\lambda \in \Omega_{\bar{k}}, \ell(\lambda) \leq N}{\bigoplus} K_{\lambda} V \otimes\left(K_{\lambda} V\right)^{*}
$$

of $A$-bicomodules of $A$ and $S_{k} E^{\vee}$.

ProOF. The first assertion is a consequence of Lemma 8.8 and Corollary 8.10. So we have $S_{k} E^{\vee} \simeq \bigoplus_{\lambda}\left(K_{\lambda} V\right)^{*} \otimes K_{\lambda} V$ as a $K$-coalgebra, and the isomorphism (8.12) is established. The category of finite dimensional $A$-bimodules can be identified with $\left(A \otimes A^{\text {op }}\right)$-mff, where ( $)^{\text {op }}$ denotes the opposite algebra. By the first assertion, we have $A \otimes A^{\mathrm{op}} \simeq \prod_{\lambda} \operatorname{End}_{K}\left(K_{\lambda} V \otimes\left(K_{\lambda} V\right)^{*}\right)$, and the decomposition (8.12) is an irreducible decomposition.

In the next section, we will discuss the $A$-bimodule (or $S E^{\vee}$-bicomodule) structure of $S_{k} E^{\vee}$ over an arbitrary commutative ring $R$ and $q \in R^{\times}$.

9. Quantum determinants and the straightening formulas. Throughout this section, $V$ and $E$ are as in $\S 7$. Let $M \in \mathrm{cff}-S E^{\vee}$. Then we have the coefficient map $\mathrm{cf}_{M}: M^{*} \otimes M \rightarrow S E^{\vee}$ (see $\S 3$ ). Since $\mathrm{cf}_{M}$ is a homomorphism of coalgebras, $M^{*} \otimes M$ is an $S E^{\vee}$-bicomodule and $\mathrm{cf}_{M}$ is a homomorphism of $S E^{\vee}$-bicomodules. The $S E^{\vee}$-bicomodule $M^{*} \otimes M$ agrees with the tensor product of the left $S E^{\vee}$-comodule $M^{*}$ and the right $S E^{\vee}$-comodule $M$. Since the counit $\bar{e}$ of $M^{*} \otimes M$ is nothing but ev : $M^{*} \otimes M \rightarrow R$, the map $(1 \otimes \mathrm{ev}) \circ(\omega \otimes 1): M^{*} \otimes M \rightarrow S E^{\vee}$ agrees with $\mathrm{cf}_{M}$. It follows that the diagrams

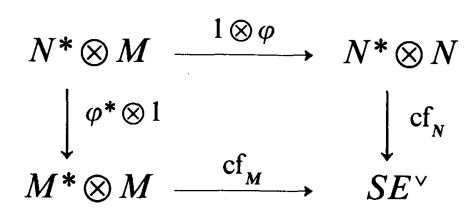

and

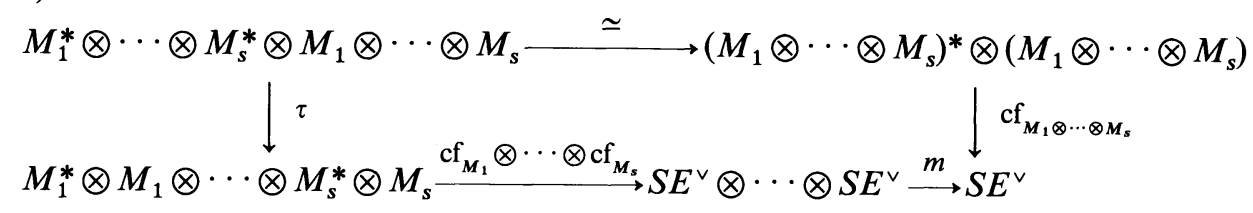


are commutative for $M, N, M_{1}, \ldots, M_{s} \in \operatorname{cff}-S E^{\vee}$ and $\varphi \in \operatorname{cff}-S E^{\vee}(M, N)$, where $\tau$ is an appropriate trivial twisting.

In what follows, we identify $\bigwedge^{t} V$ with $(\bigwedge V)^{\dagger}$ via the left $S E^{\vee}$-equivariant graded YB bialgebra isomorphism $\Phi^{\dagger}: \bigwedge^{t} V \rightarrow(\bigwedge V)^{\dagger}$, where $\Phi: \bigwedge V \rightarrow\left(\bigwedge^{t} V\right)^{\dagger}$ is the map which appears in Proposition 5.2. So $\bigwedge_{k}{ }^{t} V(k \geq 0)$ is identified with $\left(\bigwedge_{k} V\right)^{*}$. More generally, we identify $\bigwedge_{\lambda}{ }^{t} V$ with $\left(\bigwedge_{\lambda} V\right)^{*}$ via the isomorphism

$$
\bigwedge_{\lambda}{ }^{t} V \stackrel{\Phi_{\lambda_{1}}^{*} \otimes \cdots \otimes \Phi_{\lambda_{s}}^{*}}{\longrightarrow}\left(\bigwedge_{\lambda_{1}} V\right)^{*} \otimes \cdots \otimes\left(\bigwedge_{\lambda_{s}} V\right)^{*} \simeq\left(\bigwedge_{\lambda} V\right)^{*}
$$

for $\lambda=\left(\lambda_{1}, \ldots, \lambda_{s}\right) \in \Omega^{+}$.

Definition 9.1. For $k \geq 0$, the coefficient map $\operatorname{cf}_{\bigwedge_{k} V}: \bigwedge_{k}{ }^{t} V \otimes \bigwedge_{k} V \rightarrow S_{k} E^{\vee}$ is called the determinant map, and is denoted by $\operatorname{det}_{k}$. For $\lambda=\left(\lambda_{1}, \ldots, \lambda_{s}\right) \in \Omega_{k}^{+}$, $\mathrm{cf}_{\bigwedge_{\lambda} V}: \bigwedge_{\lambda}{ }^{t} V \otimes \bigwedge_{\lambda} V \rightarrow S_{k} E^{\vee}$ is denoted by $\operatorname{det}_{\lambda}$.

By the commutativity of (9.2), $\operatorname{det}_{\lambda}$ is the composite map

$$
\begin{gathered}
\bigwedge_{\lambda}{ }^{t} V \otimes \bigwedge_{\lambda} V \stackrel{{ }^{\tau}}{\longrightarrow} \bigwedge_{\lambda_{1}}{ }^{t} V \otimes \bigwedge_{\lambda_{1}} V \otimes \cdots \otimes \bigwedge_{\lambda_{s}}{ }^{t} V \otimes \bigwedge_{\lambda_{s}} V \\
\stackrel{\operatorname{det}_{\lambda_{1}} \otimes \cdots \otimes \operatorname{det}_{\lambda_{s}}}{\longrightarrow} S_{\lambda} E^{\vee} \stackrel{m}{\longrightarrow} S_{k} E^{\vee} .
\end{gathered}
$$

Note that $\operatorname{det}_{\lambda}$ is an $S E^{\vee}$-bicomodule homomorphism.

LeMma 9.2. Let $k \geq 0$. Then, $\operatorname{det}_{k}$ is given by

$$
\begin{aligned}
\operatorname{det}_{k}\left(v_{i_{1}} \wedge \cdots \wedge v_{i_{k}} \otimes u_{j_{1}} \wedge \cdots \wedge u_{j_{k}}\right) & =\sum_{\sigma \in \Xi_{k}}(-q)^{\ell(\sigma)} x_{i_{\sigma 1} j_{1}} \cdots x_{i_{\sigma k} j_{k}} \\
& =\sum_{\sigma \in \subseteq_{k}}(-q)^{\ell(\sigma)} x_{i_{1} j_{\sigma 1}} \cdots x_{i_{k} j_{\sigma k}}
\end{aligned}
$$

for $1 \leq i_{1}<\cdots<i_{k} \leq N$ and $1 \leq j_{1}<\cdots<j_{k} \leq N$. The map $\operatorname{det}_{\left(1^{k}\right)}:\left({ }^{t} V\right)^{\otimes k} \otimes V^{\otimes k} \rightarrow S_{k} E^{\vee}$ is surjective.

ProOF. It is easy to see that $\operatorname{det}_{1}:{ }^{t} V \otimes V \rightarrow E^{\vee}$ is nothing but the identification $V^{*} \otimes V \rightarrow E^{\vee}$. By (9.3), $\operatorname{det}_{\left(1^{k}\right)}$ is surjective, and is given by

$$
\operatorname{det}_{\left(1^{k}\right)}\left(\left(v_{a_{1}} \otimes \cdots \otimes v_{a_{k}}\right) \otimes\left(u_{b_{1}} \otimes \cdots \otimes u_{b_{k}}\right)\right)=x_{a_{1} b_{1}} \cdots x_{a_{k} b_{k}}
$$

for $1 \leq a_{1}, \ldots, a_{k}, b_{1}, \ldots, b_{k} \leq N$. Since $m:\left({ }^{t} V\right)^{\otimes k} \rightarrow \bigwedge_{k}{ }^{t} V\left(\right.$ resp. $\left.\Delta: \bigwedge_{k}{ }^{t} V \rightarrow\left({ }^{t} V\right)^{\otimes k}\right)$ is the dual of $\Delta: \bigwedge_{k} V \rightarrow V^{\otimes k}$ (resp. $m: V^{\otimes k} \rightarrow \bigwedge_{k} V$ ), the diagram 


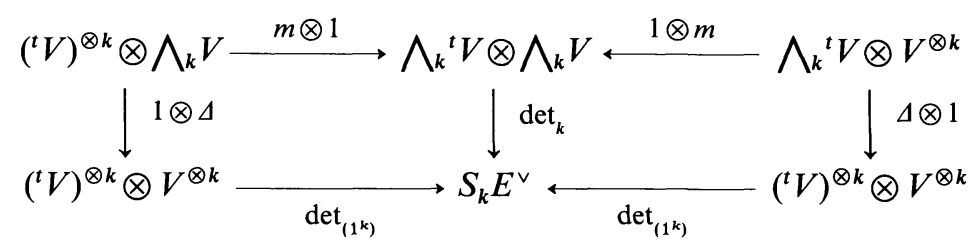

is commutative, thanks to the commutativity of the diagram (9.1). By the commutativity of the left (resp. right) square in (9.6) and Lemma 5.3, the first (resp. the second) equality in (9.4) holds.

Lemma 9.3 (The Laplace expansion). Let $i, j \geq 0$. Then, the following diagram commutes.

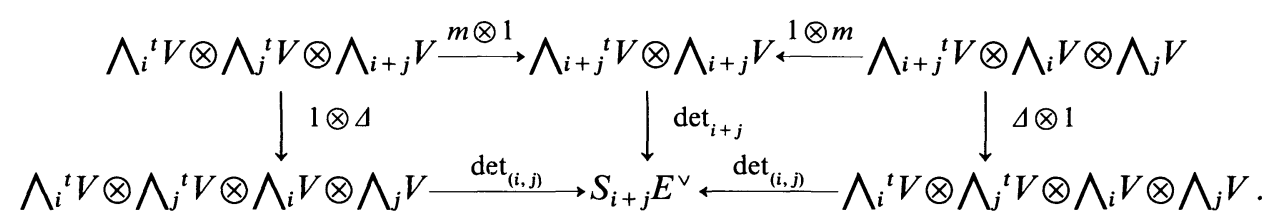

Proof. Since $m: \bigwedge_{i}{ }^{t} V \otimes \bigwedge_{j}{ }^{t} V \rightarrow \bigwedge_{i+j}{ }^{t} V$ (resp. $\Delta: \bigwedge_{i+j}{ }^{t} V \rightarrow \bigwedge_{i}{ }^{t} V \otimes \bigwedge_{j}{ }^{t} V$ ) is identified with the dual of $\Delta: \bigwedge_{i+j} V \rightarrow \bigwedge_{i} V \otimes \bigwedge_{j} V\left(\right.$ resp. $\left.m: \bigwedge_{i} V \otimes \bigwedge_{j} V \rightarrow \bigwedge_{i+j} V\right)$, the lemma follows from the commutativity of (9.1).

Lemma 9.4 (cf. [4, Proposition III.1.1]). Let $k \geq 0$ and $\lambda \in \Omega_{k}^{-}$. For $\mu \in S_{\square}(\lambda)$, the following diagram commutes:

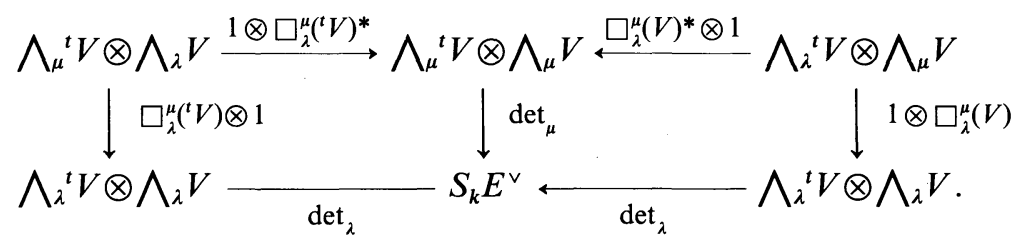

This follows immediately from the commutativity of (9.1).

Let $M, N \in \mathrm{cff}-S E^{\vee}$, and $\varphi: M \rightarrow N$ be an $S E^{\vee}$-isomorphism. Then, we have $\operatorname{Im~} \mathrm{cf}_{M}=\operatorname{Im~cf}_{N}$ by the commutativity of (9.1).

Lemma 9.5. Let $\lambda=\left(\lambda_{1}, \ldots, \lambda_{s}\right) \in \Omega^{+}$and $\sigma \in \mathfrak{S}_{s}$. Then, we have $\bigwedge_{\lambda} V \simeq \bigwedge_{\sigma \lambda} V$ and $\operatorname{Im} \operatorname{det}_{\lambda}=\operatorname{Im} \operatorname{det}_{\sigma \lambda}$, where $\sigma \lambda=\left(\lambda_{\sigma^{-1}}, \ldots, \lambda_{\sigma^{-1}}\right)$. In particular, we have $\operatorname{Im} \operatorname{det}_{\lambda}=$ $\operatorname{Im} \operatorname{det} \tilde{\tilde{\lambda}^{\sim}}$.

Proof. It suffices to show that $\bigwedge_{\lambda} V \simeq \bigwedge_{\sigma \lambda} V$ by the observation above. But this is obvious, since the isomorphism $\psi_{\wedge}(\sigma):(\bigwedge V)^{\otimes s} \rightarrow\left(\bigwedge^{\otimes}\right)^{\otimes s}$ maps $\bigwedge_{\lambda} V$ onto $\bigwedge_{\sigma \lambda} V$.

Let $\lambda \geq 0$ and $\lambda \in \Omega_{k}^{-}$. We define:

$$
M(\lambda)=\sum_{\mu \in \Omega_{\bar{k}}^{-}, \mu \geq \lambda} \operatorname{Im}_{\operatorname{det}_{\mu}} \subset S_{k} E^{\vee}
$$


and

$$
\dot{M}(\lambda)=\sum_{\mu \in \Omega_{k}, \mu>\lambda} \operatorname{Im~det}_{\mu} \subset M(\lambda) .
$$

Note that $M(\lambda)$ and $\dot{M}(\lambda)$ are subbicomodules of $S_{k} E^{\vee}$, and that $\lambda, \mu \in \Omega_{k}^{-}, \mu \geq \lambda$ imply $M(\mu) \subset M(\lambda)$. By Lemma 9.2, we have $S_{k} E^{\vee}=M\left(\left(1^{k}\right)\right)$. Hence, we have a filtration

$$
S_{k} E^{\vee}=M\left(\left(1^{k}\right)\right) \supset M\left(\left(2,1^{k-2}\right)\right) \supset \cdots \supset M((k)) \supset 0
$$

of $S_{k} E^{\vee}$.

THEOREM 9.6 (Straightening formula). Let $k \geq 0$ and $\lambda \in \Omega_{k}^{-}$. Then we have a unique isomorphism of $S E^{\vee}$-bicomodules $\Theta_{\lambda}: L_{\lambda}{ }^{t} V \otimes L_{\lambda} V \rightarrow M(\lambda) / \dot{M}(\lambda)$ which makes the following diagram commutative:

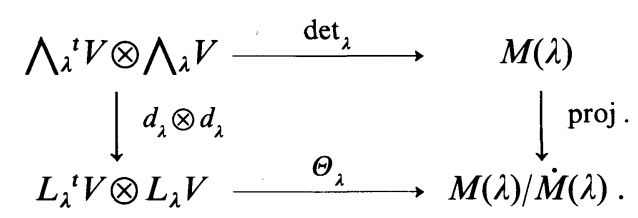

Hence, the associated graded object of the filtration $\{M(\lambda)\}_{\lambda \in \Omega_{\bar{k}}}$ is $\oplus_{\lambda \in \Omega_{\bar{k}}} L_{\lambda}{ }^{t} V \otimes L_{\lambda} V$. The set $\left\{\operatorname{det}_{\lambda}\left(\xi_{S} \otimes \xi_{T}\right) \mid \lambda \in \Omega_{k}^{-}, S \in \mathrm{St}_{\lambda} Y, T \in \mathrm{St}_{\lambda}{ }^{t} Y\right\}$ is a free basis of $S_{k} E^{\vee}$, where ${ }^{t} Y=\left\{v_{1}<\cdots<v_{N}\right\}$ is the dual basis of $Y=\left\{u_{1}<\cdots<u_{N}\right\}$.

Proof. Let $v \in S_{\square}(\lambda)$. Then, we have $\operatorname{Im} \operatorname{det}_{v}=\operatorname{Im} \operatorname{det}_{\tilde{v}^{\sim}}$ by Lemma 9.5. Since $\tilde{v}^{\sim}>\lambda$, we have $\operatorname{Im} \operatorname{det}_{v} \subset \dot{M}(\lambda)$. By Lemma 9.4, we have $\operatorname{det}_{\lambda}\left(\operatorname{Im}\left(1 \otimes \square_{\lambda}^{v}\right)\right) \subset \dot{M}(\lambda)$ and $\operatorname{det}_{\lambda}\left(\operatorname{Im}\left(\square_{\lambda}^{v} \otimes 1\right)\right) \subset \dot{M}(\lambda)$. Hence, we have $\operatorname{det}_{\lambda}\left(\operatorname{Im}\left(\square_{\lambda} \otimes 1\right)+\operatorname{Im}\left(1 \otimes \square_{\lambda}\right)\right) \subset \dot{M}(\lambda)$. By Theorem 6.19, we have $\operatorname{Ker}\left(d_{\lambda} \otimes d_{\lambda}\right)=\operatorname{Im}\left(\square_{\lambda} \otimes 1\right)+\operatorname{Im}\left(1 \otimes \square_{\lambda}\right)$. Hence, we have a unique induced map $\Theta_{\lambda}: L_{\lambda}{ }^{t} V \otimes L_{\lambda} V \rightarrow M(\lambda) / \dot{M}(\lambda)$ which makes (9.10) commutative. By the definition of $M(\lambda)$ and $\dot{M}(\lambda)$, the composite map $\operatorname{proj}_{\circ} \operatorname{det}_{\lambda}$ is surjective, and so is $\Theta_{\lambda}$. We set $\Gamma(\lambda)=\left\{\operatorname{det}_{\mu}\left(\xi_{S} \otimes \xi_{T}\right) \mid \mu \in \Omega_{k}^{-}, \mu \geq \lambda, S \in \mathrm{St}_{\mu} Y, T \in \mathrm{St}_{\mu}{ }^{t} Y\right\}$ for $\lambda \in \Omega_{k}^{-}$. By induction on $\lambda$, it is easy to see that $\Gamma(\lambda)$ generates $M(\lambda)$, thanks to Theorem 6.19. On the other hand, we have rank $S_{k} E^{\vee}=\sum_{\lambda \in \Omega_{\bar{k}}}\left(\#\left(\mathrm{St}_{\lambda} Y\right)\right)^{2}=\# \Gamma\left(1^{k}\right)$. To prove this, we may assume that $R=C(q)$, and this case is a consequence of Corollary 8.11. It follows that $\Gamma\left(1^{k}\right)$ is linearly independent, and $\Gamma(\lambda)$ is a free basis of $M(\lambda)$ for $\lambda \in \Omega_{k}^{-}$. Hence, $\left\{\Theta_{\lambda}\left(d_{\lambda} \xi_{S} \otimes d_{\lambda} \xi_{T}\right) \mid S \in \mathrm{St}_{\lambda} Y, T \in \mathrm{St}_{\lambda}{ }^{t} Y\right\}$ is linearly independent, and $\Theta_{\lambda}$ is injective for $\lambda \in \Omega_{k}^{-}$. Since $\operatorname{det}_{\lambda}$, proj and $d_{\lambda} \otimes d_{\lambda}$ are $S E^{\vee}$-bicomodule homomorphisms, so is $\Theta_{\lambda}$. The theorem is now clear.

Let $N \in \mathscr{Q}$, and $v \in \Omega^{+}$with $\ell(v) \leq N$. We define ${ }_{v} N:=N t^{*(v)} \subset N$. By Lemma 7.8 and its proof, we have ${ }_{v} N \simeq\left(N_{v}^{*}\right)^{*} \simeq\left(\operatorname{tr}_{\#} N\right)_{v}$. For $\lambda \in \Omega_{k}^{-}$with $\ell(\tilde{\lambda})=\lambda_{1} \leq N$, we set $c^{\prime}(\lambda):=v_{1} \wedge \cdots \wedge v_{\lambda_{1}} \otimes \cdots \otimes v_{1} \wedge \cdots \wedge v_{\lambda_{N}} \in \bigwedge_{\lambda}{ }^{t} V$. It is easy to see that $c^{\prime}(\lambda) \in \tilde{\lambda}\left(\bigwedge_{\lambda}{ }^{t} V\right)$, and $d_{\lambda}\left(c^{\prime}(\lambda)\right)$ generates $\tilde{\lambda}\left(L_{\lambda}{ }^{t} V\right)=\left(K_{\tilde{\lambda}} V\right)_{\hat{\lambda}}^{*} \simeq R$. We set 


$$
L_{q}^{\lambda}:=\operatorname{det}_{\lambda}\left(c(\lambda) \cdot R \otimes \bigwedge_{\lambda} V\right) \subset S_{k} E^{\vee} .
$$

This definition is due to Taft and Towber [37, §3]. Clearly, $L_{q}^{\lambda}$ is a quotient $S_{k} E^{\vee}$-comodule of $\bigwedge_{\lambda} V$ via the surjection

$$
\bigwedge_{\lambda} V \stackrel{\sim}{\longrightarrow} c^{\prime}(\lambda) R \otimes \bigwedge_{\lambda} V \stackrel{\operatorname{det}_{\lambda}}{\longrightarrow} L_{q}^{\lambda} V
$$

and is a subcomodule of $S_{k} E$.

Proposition 9.7. Let $\lambda \in \Omega_{k}^{-}$and $\ell(\lambda) \leq N$. Then we have $L_{q}^{\lambda} \simeq L_{\lambda} V$ as an $S_{k} E^{\vee}$-comodule.

Proof. For $\mu \in \Omega_{k}^{-}$with $\ell(\lambda) \leq N$, we have $\mu>\lambda \Leftrightarrow \tilde{\mu} \prec \tilde{\lambda}$ (see [25, (1.11)]). Hence, $\mu>\lambda$ implies $\tilde{\mu} \nsucceq \tilde{\lambda}$. By Theorem 7.12, we have ${ }_{\lambda}\left(L_{\mu}{ }^{t} V\right)=\left(K_{\tilde{\mu}} V\right)_{\tilde{\lambda}}^{*}=0$ for $\mu \in \Omega_{k}^{-}$ with $\ell(\mu) \leq N$ and $\mu>\lambda$. By Theorem 9.6, we have $\tilde{\lambda} \dot{M}(\lambda)=0$. Hence, the projection proj: $\tilde{\lambda} M(\lambda) \rightarrow \tilde{\lambda}(M(\lambda) / \dot{M}(\lambda))$ is an isomorphism. Since $L_{q}^{\lambda}$ is contained in $\tilde{\lambda} M(\lambda)$, we have

$$
\begin{aligned}
L_{q}^{\lambda} & =\operatorname{proj} \circ \operatorname{det}_{\lambda}\left(c^{\prime}(\lambda) \cdot R \otimes \bigwedge_{\lambda} V\right)=\Theta_{\lambda} \circ\left(d_{\lambda} \otimes d_{\lambda}\right)\left(c^{\prime}(\lambda) \cdot R \otimes \bigwedge_{\lambda} V\right) \\
& =d_{\lambda}\left(c^{\prime}(\lambda)\right) \cdot R \otimes L_{\lambda} V \simeq L_{\lambda} V .
\end{aligned}
$$

\section{REFERENCES}

[1] E. Abe, Hopf algebras, Cambridge Tracts in Math. 74, Cambridge Univ. Press, 1980.

[2] K. AKIn AND D. A. BuchsBaum, Characteristic-free representation theory of the general linear group, Adv. in Math. 58 (1985), 149-200.

[ 3 ] K. Akin And D. A. Buchsbaum, Characteristic-free representation theory of the general linear group II Homological considerations, Adv. in Math. 72 (1988), 171-210.

[4] K. Akin, D. A. Buchsbaum and J. Weyman, Schur functors and Schur comlexes, Adv. in Math. 44 (1982), 207-278.

[ 5] G. M. Bergman, The diamond lemma for ring theory, Adv. in Math. 29 (1978), 178-218.

[6] C. CURTIS AND I. Reiner, Representation theory of finite groups and associative algebras, Pure and Appl. Math., vol. 11, Interscience, New York, 1962; 2nd ed., 1966.

[7] V. G. DRIN'FELD, Quantum groups, Proc. ICM, Berkeley, 1986.

[8] E. DATE, M. Jimbo and T. Miwa, Representations of $U_{q}(\operatorname{gl}(n, C))$ at $q=0$ and the Robinson-Shensted correspondence, Memorial Volume for Vadim Kniznik, Physics and Mathematics of Strings, World Scientific, Singapore, 1990, 185-211.

[9] P. Deligne and J. Milne, Tannakian categories, Lecture Notes in Math. 900, Springer-Verlag, Berlin Heidelberg New York, 1982, 101-228.

[10] R. DIPPER AND G. JAMES, $q$-tensor space and $q$-Weyl modules, preprint.

[11] P. Doubilet, G.-C. Rota AND J. STEIN, Foundations of combinatorics. IX. Combinatorial methods in invariant theory, Stud. Appl. Math. 43 (1971), 1020-1058.

[12] L. D. Faddeev, N. Yu. Reshetikhin and L. A. Takhtajan, Quantization of Lie groups and Lie algebras, Algebra and Analysis 1 (1989), 178-206 (English translation: Leningrad Math. J. 1 (1990), 193-225). 
[13] J. A. Green, Polynomial representations of $G L_{n}$, Lecture Notes in Math. 830, Springer-Verlag, Berlin Heidelberg New York, 1980.

[14] A. Gyoja, A q-analogue of Young symmetrizer, Osaka J. Math. 23 (1986), 841-852.

[15] A. Gyoja ANd K. Uno, On the semisimplicity of Hecke algebras, J. Math. Soc. Japan 41 (1989), 75-79.

[16] T. HiBI, Distributive lattices, affine semigroup rings and algebras with straightening laws, in Advanced Studies in Pure Math. 11, Kinokuniya, Tokyo and North-Holland, Amsterdam, 1987, 93-109.

[17] T. HayASHI, Quantum deformation of classical groups, Publ. Res. Inst. Math. Sci., Kyoto Univ. 28 (1992), 57-81.

[18] M. Jimbo, A $q$-difference analogue of $U(\mathfrak{g})$ and the Yang-Baxter equation, Lett. in Math. Phys. 10 (1985), 63-69.

[19] M. Jimbo, Quantum $R$ matrix related to the generalized Toda system: an algebraic approach, Lecture Notes in Phys. 246, Springer-Verlag, Berlin Heidelberg New York, 1986, 335-361.

[20] M. Jimbo, A $q$-analogue of $U(\mathrm{gl}(N+1))$, Hecke algebra and the Yang-Baxter equation, Lett. in Math. Phys. 11 (1986), 247-252.

[21] P. P. Kulish, N. Yu. Reshetikhin and E. K. Sklyanin, Yang-Baxter equation and representation theory. I, Lett. Math. Phys. 5 (1981), 393-403.

[22] P. P. Kulish and E. K. Sklyanin, Quantum spectral transform method. Recent developments, Integrable quantum field theories, Lecture Notes in Physics 151, Springer-Verlag, Berlin Heidelberg New York, 1981, 61-119.

[23] G. LuszTig, Modular representations and quantum groups, Contemp. Math. 82, Amer. Math. Soc., Providence, R. I., 1989, 59-77.

[24] V. V. LyUbaSHENKo, Hopf algebras and vector-symmetries, Uspekhi Mat. Nauk, 41-5 (1986), 185-186 (in Russian).

[25] I. G. Macdonald, Symmetric functions and Hall polynomials, Oxford Univ. Press, 1979.

[26] YU. I. MANIN, Some remarks on Koszul algebras and quantum groups, Ann. Inst. Fourier, Grenoble 37 (1987), 191-205.

[27] YU. I. MANIN, Multiparametric quantum deformation of the general linear supergroup, Commun. Math. Phys. 123 (1989), 163-175.

[28] D. G. NorthCott, Multilinear algebra, Cambridge University Press, 1984.

[29] M. Noumi, H. Yamada ANd K. Mimachi, Finite dimensional representations of the quantum group $G L_{q}(n)$ and the zonal spherical functions on $U_{q}(n-1) \backslash U_{q}(n)$, preprint.

[30] B. Parshall and J. Wang, Quantum linear groups, Memoirs of the Amer. Math. Soc. 439 (1991).

[31] P. Podles, Quantum spheres, Lett. in Math. Phys. 14 (1987), 193-202.

[32] S. B. Priddy, Koszul resolutions, Trans. Amer. Math. Soc. 152-1 (1970), 39-60.

[33] N. YU. ReshetikHIN, Quantum universal enveloping algebras, the Yang-Baxter equation and invariants of links. I, preprint.

[34] M. Sweedler, Hopf algebras, W. A. Benjamin, New York, 1969.

[35] M. TAKEUCHI, Matric bialgebras and quantum groups, Israel J. of Math. 72 (1990), 232-251.

[36] T. TANISAKI, Finite dimensional representations of quantum groups, Osaka J. of Math. 28 (1991), 37-54.

[37] E. TAFT AND J. TowBER, Quantum deformation of flag schemes and Grassmann schemes I A $q$-deformation of the shape-algebra for $G L(n)$, J. Algebra 142 (1991), 1-36.

[38] V. G. TuraEv, The Yang-Baxter equation and invariant of links, Invent. Math. 92 (1988), 527-553.

[39] K. Ueno, T. Takebayashi, Y. Shibukawa, Gelfand-Tsetlin basis for representations of the quantum group $G L_{q}(N+1)$ and the basic quantum affine space, preprint.

[40] S. L. Woronowicz, Compact matrix pseudogroups, Comm. Math. Phys. 111 (1987), 613-665. 


\section{QUANTUM MULTILINEAR ALGEBRA}

Department of Mathematics

SCHOOL OF SCIENCE

NAGOYA UNIVERSITY

NAGOYA 464

JAPAN 
